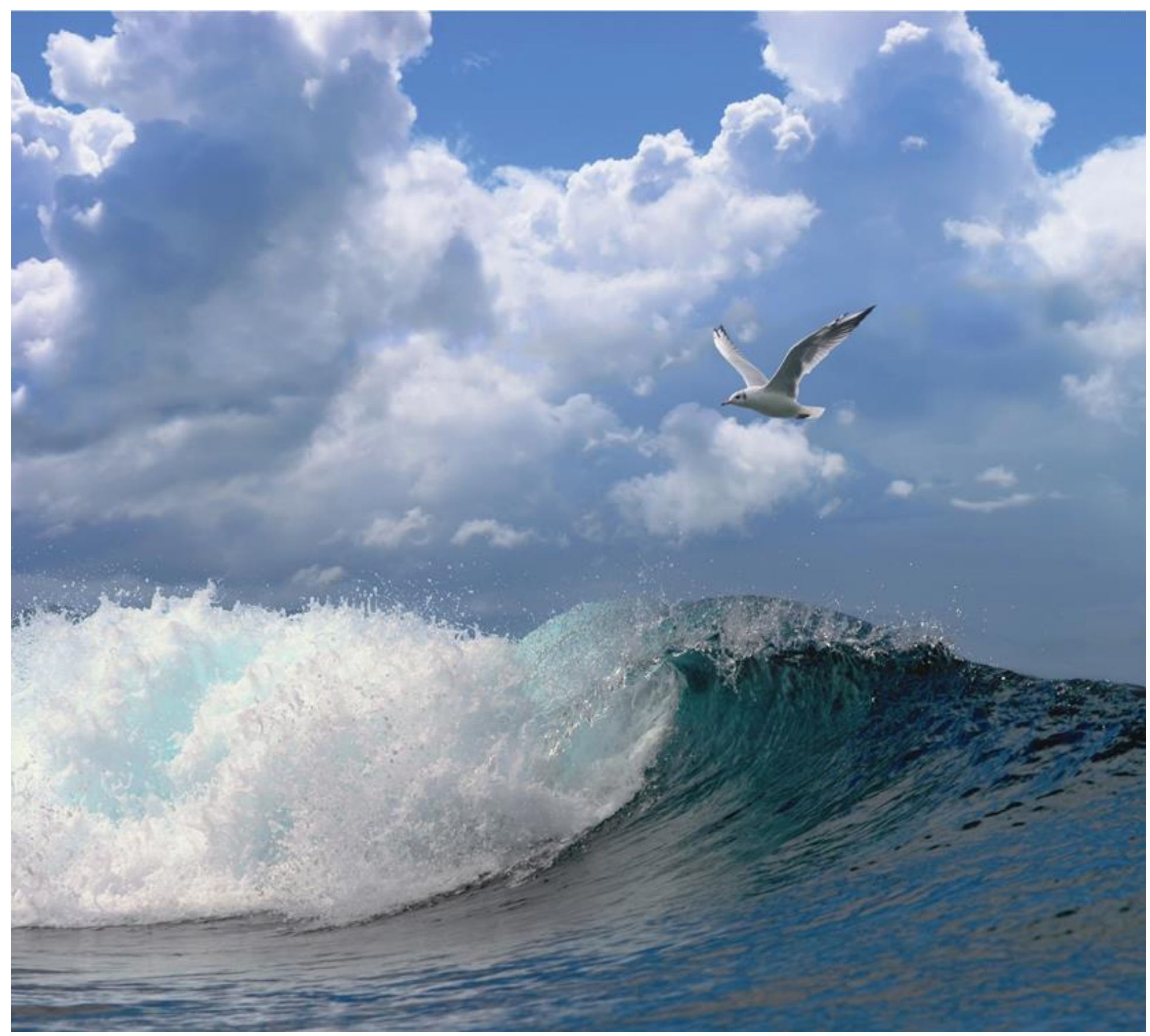

Desktop study on autecology and productivity of European lobster (Homarus gammarus, $L$ ) in offshore wind farms 


\section{Desktop study on autecology and productivity of European lobster (Homarus gammarus, L) in offshore wind farms}
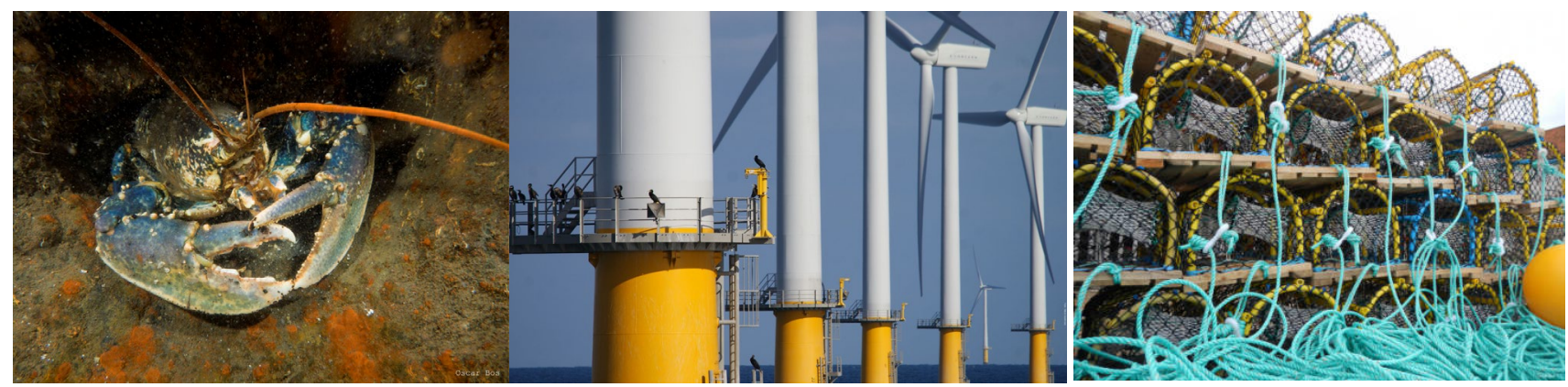

KB-30: Resource Use Efficiency (project no. KB-30-002-011). 
Keywords: Homarus gammarus, European lobster, production potential, offshore wind farm, North Sea, Dutch NCP

Client: Wageningen Research Investment Programme Resource Use Efficiency

KB-30-002-011

Attn.: Secretary RUE projects

H. van der Kolk

Foundation Wageningen Research

P.O. Box 17

6700 AA Wageningen

The Netherlands

This report can be downloaded for free from https://doi.org/10.18174/466861

Wageningen Marine Research provides no printed copies of reports

Wageningen Marine Research is ISO 9001:2015 certified.

Photo cover: Foto 1 en 3 Oscar Bos, Foto 2 Steve Geelhoed

(C) Wageningen Marine Research

Wageningen Marine Research institute of Stichting Wageningen Research is registered in the Dutch traderecord nr. 09098104, BTW nr. NL 806511618
The Management of Wageningen Marine Research is not responsible for resulting damage, as well as for damage resulting from the application of results or research obtained by Wageningen Marine Research, its clients or any claims related to the application of information found within its research. This report has been made on the request of the client and is wholly the client's property. This report may not be reproduced and/or published partially or in its entirety without the express written consent of the client. 


\section{Contents}

$\begin{array}{lr}\text { Summary } & 5\end{array}$

$\begin{array}{llr}1 & \text { Introduction } & 7\end{array}$

$\begin{array}{lll}1.1 & \text { Problem definition } & 7\end{array}$

1.1.1 Target group and knowledge $\quad 8$

1.2 Objectives of the project 9

$2 \quad$ Literature review on lobster $r$

2.1 Description $\quad 10$

$\begin{array}{lll}2.1 .1 & \text { Age } & 10\end{array}$

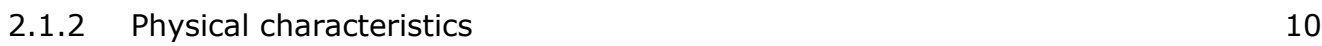

$\begin{array}{ll}2.2 & \text { Distribution }\end{array}$

2.3 Landings 14

2.3.1 Regulations and management on landings $\quad 14$

2.3.2 Landings 14

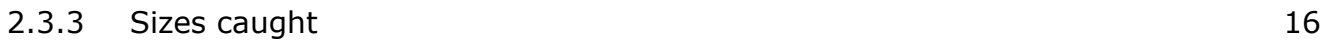

2.4 Biology and habitat $\quad 16$

$\begin{array}{lll}2.5 & \text { Densities } & 21\end{array}$

\begin{tabular}{ll}
2.6 & Reproduction and juveniles \\
\hline
\end{tabular}

2.6.1 Mating 25

2.6.2 Pelagic larval stage $\quad 26$

2.6.3 Early settlement $\quad 26$

$\begin{array}{lll}2.6 .4 & \text { Refugia } & 27\end{array}$

$\begin{array}{lll}2.7 & \text { Diet } & 27\end{array}$

$\begin{array}{lll}2.8 & \text { Mobility } & 28\end{array}$

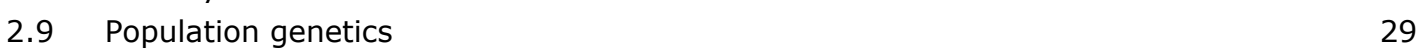

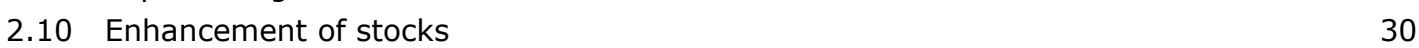

2.10.1 Habitat enhancement 31

2.10.2 Stock enhancement 31

$\begin{array}{ll}2.10 .3 \text { Food enhancement } & 32\end{array}$

$3 \quad$ Other relevant aspects for modelling 33

3.1 OWF Princess Amalia $\quad 33$

$\begin{array}{lll}3.2 & \text { Habitat enhancement through Reef Balls } & 34\end{array}$

$4 \quad$ Modelling lobster production $\quad 36$

$\begin{array}{lll}4.1 & \text { Method and parameters } & 36\end{array}$

$\begin{array}{lll}4.2 & \text { Results } & 39\end{array}$

4.2.1 DEB stand-alone $\quad 39$

$\begin{array}{lll}\text { 4.2.2 Population dynamical model } & 43\end{array}$

$\begin{array}{lll}4.3 & \text { Restocking } & 44\end{array}$

$\begin{array}{lll}4.4 & \text { Model discussion } & 47\end{array}$

$5 \quad$ Conclusions, discussion and recommendations $\quad 48$

$\begin{array}{lll}5.1 & \text { Main findings } & 48\end{array}$

$\begin{array}{lll}\text { 5.1.1 Lobster ecology } & 48\end{array}$

5.1.2 DEB modelling and lobster production $\quad 49$

$\begin{array}{lll}5.1 .3 & \text { Enhancement strategies } & 49\end{array}$

$\begin{array}{llr}5.2 & \text { Recommendations } & 50\end{array}$ 
5.2.1 Early stages $\quad 50$

$\begin{array}{lll}5.2 .2 & \text { Presence, density and habitat } & 50\end{array}$

5.2.3 Position and functioning in the food web $\quad 50$

5.2.4 Physiology and DEB modelling 50

5.2.5 Primary production and benthic-pelagic coupling 50

$\begin{array}{lll}\text { 5.2.6 Enhancement strategies } & 50\end{array}$

6 Quality Assurance

52

$\begin{array}{lr}\text { References } & \mathbf{5 2}\end{array}$

$\begin{array}{lr}\text { Justification } & \mathbf{5 8}\end{array}$

$\begin{array}{llr}\text { Annex } 1 & 59\end{array}$

$\begin{array}{llr}\text { Annex } 2 & \text { Diet composition Homarus americanus } & 61\end{array}$ 


\section{Summary}

This desk study describes the biology of the European lobster $H$. gammarus. Using the obtained data a model was developed to describe the growth of the European lobster under assumed conditions on the anti-scouring of monopiles in Dutch OWFs. One of the main questions to answer was, if, theoretically, local productivity supports the continuous harvesting of lobsters with passive fishery methods.

\section{Main findings}

\section{Lobster ecology}

The first finding is that lobster is characterised as a large top end predator. It exhibits a k-strategy life style, able to reach very old ages (e.g. an estimated 72 years). The planktonic and early benthic stages are very vulnerable and susceptible to predation. It grows slowly, taking at least four years to reach maturity in favourable conditions although in general maturity is thought to start from five years old depending on ambient conditions. Reproduction occurs in a two to three year cycle. The eggs are held on the pleopods for approximately a year until hatching. In itself the reproduction is sufficient to maintain populations at constant levels (given the high age). However, global lobster populations have suffered considerable fishing pressure and stock collapse, only recovering slowly now at some regions partly due to appropriate management (the MCRS and stock enhancement programmes, (Wickins \& Lee, 2002, Prodöhl et al., 2006, Phillips, 2013)).

In principle European lobsters have a high enough mobility to colonize the entire North Sea using wrecks, hard substrates etcetera as stepping stones (Krone and Schröder, 2011). Yet, unexpected differentiated genetic clusters occur which implies that at a North Sea scale realized mobility is more limited than potential mobility. Apparently the exchange is less than based on theoretical estimates of mobility.

The limited exchange implies that when exploiting lobsters at OWFs an exploitation management plan should be established based on the local OWF population dynamics and the presence of lobsters nearby on the soft sediments. Also stock enhancement using larvae or juveniles from local populations might be an additional option. The seafloor at the OWF could be an issue in choosing at which size to release juvenile lobsters since the earliest benthic stages can dig their holes. When the seafloor is more sandy than solid mud, early stages are entirely depending on the crevices between stones and cobbles. In addition they actively harvest the walls of their residence. The more productive the seafloor bottom is (in terms of worms, amphipods etc.) the less frequent these vulnerable stages have to expose themselves to predation and currents (Lawton \& Lavalli, 1995, Jensen et al., 2000, Wickins $\&$ Lee, 2002). In this sense older and larger stages ( $>$ an estimated $15 \mathrm{~mm} \mathrm{CL}$ ) are less dependent on bottom type (and more on availability of crevices).

European lobster prefer hard substrate with crevices to hide, except for the pelagic larval stage. At younger stages they are entirely dependent on shelter in order to evade predation. At later stages they become less dependent on shelter but keep a preference for hard substrate. Crevices should have the size to accommodate the complete body and should be available for all sizes. Younger animals of European and American lobsters move to larger crevices while growing (Lawton \& Lavalli, 1995, Jensen et al., 2000, Dunnington et al., 2005, Phillips, 2013)

Taking the expected crevice size into account for OWF Prinses Amalia and OWF Luchterduinen (maximally $8 \times 8 \times 8 \mathrm{~cm}$, Table 7), theoretically there is a lack of suitable crevices for larger sized lobster in the OWFs preselected for TKI WinWind. Adding extra hard structures with crevices suitable for lobster might stimulate the presence of legal catchable lobster (at $85 \mathrm{~mm} \mathrm{CL}$, an estimated 240 mm total length, Prodhöl et al., 2006). Monitoring is needed to establish which size-classes of $H$. gammarus are present in the OWFs. A potential new habitat should be carefully designed accommodating several sizes classes of lobster, having sufficient water and oxygen refreshment and also enabling stocks of the typical food like mussels and crabs. 
Lobster densities at suitable substrates of a fished population are reported to be 1 lobster per $\sim 150 \mathrm{~m}^{2}$. American lobsters can have much higher natural densities ( 1 lobster every $6 \mathrm{~m}^{2}$ ) but one should keep in mind that this species is migratory (Dunnington et al., 2005, Geraldi et al., 2009, Phillips, 2013), which might explain that a given substrate does not have to provide food year round. In addition, they have a higher mobility so likely have a greater foraging area.

\section{DEB modelling and lobster production}

The DEB model predicts lobster growth in line with growth reported in literature, although length at age from field data shows a large variability. Based on the current model parameter setting resource production is limiting lobster growth and abundance while ensuring that lobsters reach marketable sizes. The current model setting predicts that 1 lobster can reach marketable size after 3 years, provided a stocking length at $50 \mathrm{~mm} \mathrm{CL}$. Model resource productivity was based on mussel density estimated at a limited survey of monopiles in a Dutch OWF. However, resource production might differ between monopiles, parks and temperature regimes, and higher productivity will support more lobsters and/or have a positive effect on individual growth rates.

The population model does not take recruitment into account, the reason being that a single monopile surface area is being studied and that little is known on factors determining recruitment success in wild populations, nor the geographical extent of such populations in the North Sea. In addition, no lobsters were observed at a studied wind park 4 years after operation started (Bouma and Lengkeek, 2012). This suggests that at least the chance of natural recruitment on monopiles in wind parks in the North Sea is low. However, when the expectancy is 1 lobster per monopile, changes of encounter during a scientific dive are not that high. Note that energy loss through egg production is accounted for in the model.

The modelled European lobster productivity is in line with low densities found in the field, using the OWF hard substrate and anti-scouring data from Bouma \& Lengkeek (2012). One lobster could be supported by one monopile given its anti-scouring surface area ( 1 per $\left.364 \mathrm{~m}^{2}\right)$.

\section{Enhancement strategies}

The current literature survey on lobster ecology linked observations to selected enhancement strategies for local populations (habitat, stock and food). All strategies seem to have a potential added value for European lobster production but measurements and monitoring in OWFs is the first step to describe the actual situation in OWFs. DEB modelling suggests that food availability is the first limiting factor. Therefore food enhancement (by e.g. improving quality by seeding with favoured food or improving availability by depositing discards of fisheries) can help to increase productivity. Habitat enhancement by means of extra hard substrate with extra crevices can serve to increase the amount of crevices available in OWFs, especially for larger lobsters. In addition, extra hard substrate can serve as fundament for extra biota and thereby provide food. Stock enhancement can help to surpass the vulnerable pelagic and early settlement stages (up to $\sim 15 \mathrm{~mm} \mathrm{CL}$ or even larger). It can also safeguard supply of animals since natural recruitment is low. 


\section{Introduction}

The multi-use of offshore wind farms (OWFs) on the Dutch National Continental Flat (NCP) with harvesting natural stocks and or aquaculture is becoming more and more realistic and accepted. Amongst others the passive low-impact fisheries of European lobster (Homarus gammarus) and brown crab (Cancer pagurus) has been proposed as an excellent opportunity. To that extent a TKI tender has been awarded and the project TKI Win-Wind on enabling this type of fisheries is in progress.

As a preparation for this project knowledge on the ecology of both species is necessary. This desk study will focus on the European lobster and make an overview of relevant features.

To be able to grow additional biomass ${ }^{1}$ on locations like OWFs, food availability to enable metabolism and growth of such additional biomass is essential. However, the production potential of the NCP for European lobsters and brown crabs is not yet well known, and hardly any spatial planning based on ecosystem productivity is currently considered. In general is valid for the benthos of the North Sea that quantity and quality of the primary production (PP) are important for the amount and quality of the biomass of the benthos. Also hydrodynamics are important determining availability. A benthos community dominated by suspension feeders like the Dutch Coastal zone or the German Bight is thought to represent a food saturated situation (Kröncke, 2006). Smaal et al. (2017) use food concentration (in $\mu \mathrm{g}$ Chlorophyll-a (Chl-a)/l) as a factor in habitat modelling of the European oyster (Ostrea edulis) in order to select the best suitable OWFs for pilot reef reconstruction experiments. They do not calculate maximum productivity potential of the European oyster based on available PP since there are hardly any recent measurements on PP in the Dutch NCP. The tools to support adequate spatial planning, and ecosystem based productivity (a driver for site selection and food harvest potential) are not yet available for utilisation. This information should be available to choose and optimize harvest and locations (in time and space) and also to be able to evaluate management measures in order to increase local productivity (e.g. evaluation of habitat enhancement, stock enhancement or food enhancement).

Growth and production models could yield such insights to assess the site dependent potential of ecosystem productivity and production species occurrence (where species can grow, and what production can be achieved). In this study a first step is taken to model productivity of European lobsters in OWFs. In this first inventorying study a model is selected best suited for our questions on productivity potential of lobster on the anti-scouring of one monopile in an OWF. An important question for the TKI Win-Wind project is whether it is possible and effective to enhance the populations of European lobsters in OWFs in order to increase the potential profits of harvesting. Therefore a first limited exploratory review will be performed on potential population enhancement strategies. Table 1 gives a definition of the population enhancement strategies that are commonly used for European lobsters (Jenner et al., 2000) that will be studied in this study.

\subsection{Problem definition}

It is currently unknown what the local maximum production capacity is for aquaculture in general and lobster in particular in especially OWFs and what drives or limits aquaculture production.

The added presence of hard substrates in de form of anti-scouring and monopiles in an OWF should lead to increased benthic biomass and diversity in a soft substrate dominated seafloor (van Moorsel et al., 1991, Leewis et al., 1997, van Moorsel \& Waardenburg, 2001, Bouma \& Lengkeek, 2012). There is little knowledge on the offshore carbon fluxes in general and how the carbon fluxes have changed

1 Either passively by just offering constructions to enhance settlement of desired species or actively by stimulating biomass grow by e.g. additional food. 
within the food web after addition of hard substrates. The potential maximum carrying capacity for aquaculture stocks is consequently also undefined. Information is needed to assess maximum aquaculture production and driving forces thereof in time and space in order to make substantiated management choices in allocation of OWFs for multi-use purposes such as aquaculture. These insights can also aid in selecting the appropriate tools for the potential improvement of local populations of European lobsters (e.g. enhancement of habitat, stock or food). Since assessing production capacity from a holistic point of view is an extensive research question, a stepwise approach is necessary. This desk study represents a first step in this approach (a simplified production capacity estimation model).

\subsubsection{Target group and knowledge}

Target groups: are governments as area managers, aquaculture companies, OWF operators and fishermen being potential users of the knowledge to optimise their business case.

Knowledge: the project will lead to knowledge and tools to manage and optimise multi-use of OWFs aiming at aquaculture in general and lobster and brown crab in particular.

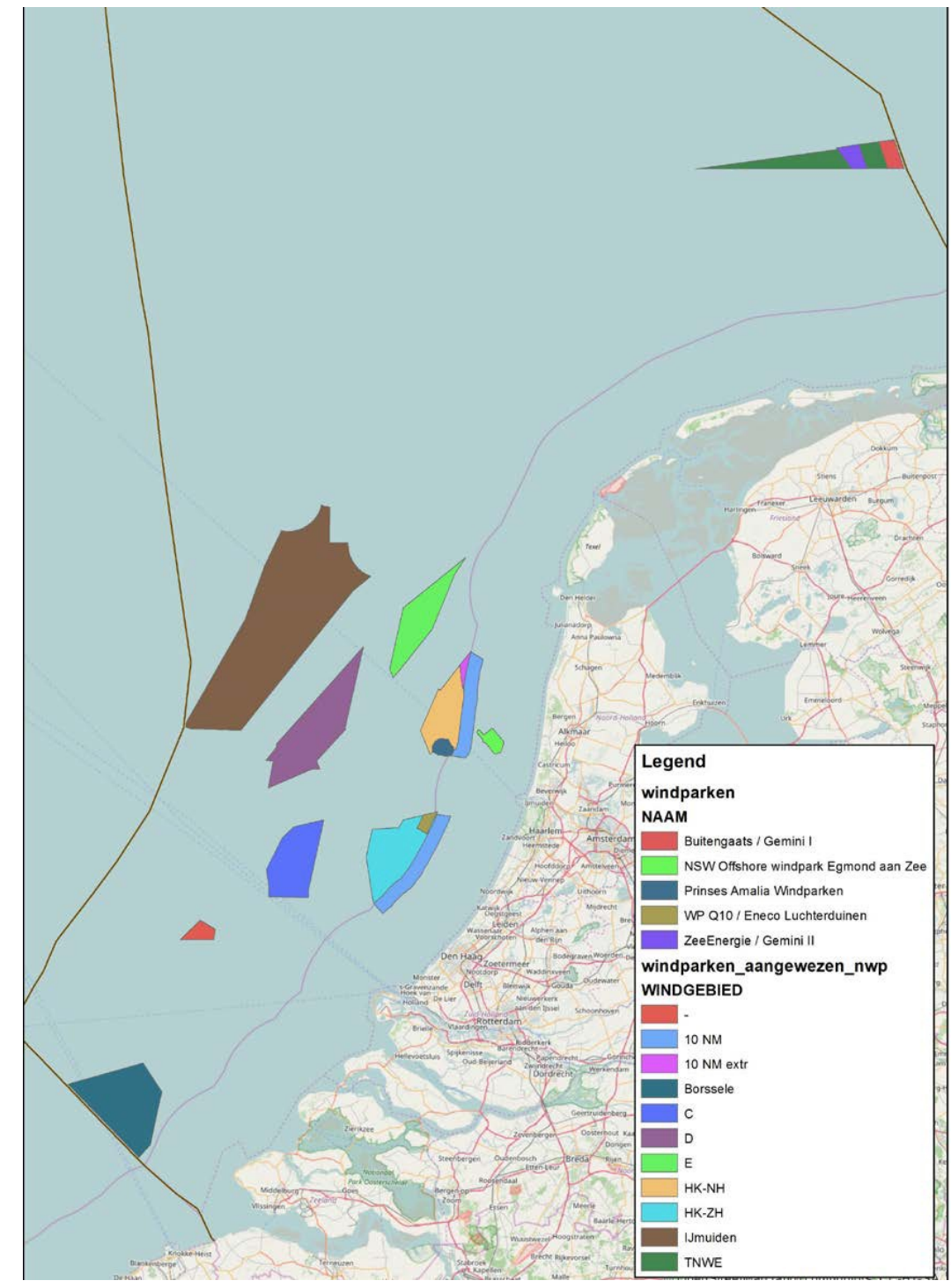

Figure 1. Map of the locations of existing and planned offshore wind farms (OWFs) in the Dutch section of the North Sea (DSC: Dutch Continental Shelf). The existing OWFs are the first names and colours given in the legend. HK-NH: Hollandse Kust Noord-Holland. HK-ZH: Hollandse Kust Zuid-Holland (fig. from Smaal et al., 2017). 


\subsection{Objectives of the project}

The objective of the project is to generate management information on the potential of aquaculture in wind parks in the North Sea:

1. This study will describe the general ecology and life history of the European lobster (H. gammarus) from which suggestions will be derived if and which population enhancement strategies can be potentially successful to promote production.

2. A first, limited exploratory review will be performed on potential population enhancement strategies (Table 1).

3. The literature study obtains the necessary data to support the (already existing but adapted) production capacity estimation model.

4. A already existing DEB model will be adapted and developed within a population dynamical framework to study the production capacity of lobster at a monopile.

5. The productivity of lobsters will be determined with the developed model fed with resource levels based on encountered biomass data on antiscouring of OWF Prinses Amalia (Bouma and Lengkeek, 2012).

6. The stock enhancement strategy will have a first exploratory test with the production capacity estimation model.

Table 1. Definitions of habitat enhancement, stock enhancement and food enhancement.

1. Habitat enhancement: E.g. pre-adult and adult lobsters are reef-obligate and select sites that supply sufficient food and oxygen and shelter from currents and predation. The brown crab exploits a broad-range of environments, ranging from soft muds into which it can hide or dig for food to hard, rocky substrata where it exploits, and seeks shelter, in crevices. (Linley et al., 2008). Next to shelter, additional habitat will yield extra food possibilities.

Reef type habitat can be improved by improving the texture of the anti-scouring construction (Lengkeek et al., 2017) or by adding artificial reef structures outside the anti-scouring zone (with specific structures and features, e.g. Jensen et al., 2000, Linley et al., 2008, Buck et al., 2017). Both options have their disadvantages: anti scouring protection has specific design criteria thereby limiting the possibilities for optimising design to meet species preferences. Adding new and extra structures outside anti-scouring locations obliges both Water-act permits and the removal of the structures on decommissioning the park (Lengkeek et al., 2017). A first exploratory literature review will be given on possibilities and applicability for the specific Dutch case in order to support management decisions on the next pilot phase.

2. Stock enhancement: is an option to increase the natural occurring number of brown crabs or preferably the more profitable lobster. Stock enhancement beholds the release of juvenile lobsters (either larvae, post larva or older stages) that have been reared in hatcheries thereby increasing survival of the vulnerable stages. Release of reared individuals enables tagging and thereby following individual growth in time and other quantitative evaluation of lobster release programmes (Jensen et al., 2000, Prodöhl et al., 2006, Cornwall National Lobster Hatchery website ${ }^{2}$ ). For the Oosterschelde ideas exist to initiate a local hatchery. Risks exist in the sense that e.g. the Oosterschelde populations represent an isolated distinct genetic group. Furthermore the released recruits i) represent also a specific (favoured) genetic pre-selection ii) that has not been selected (survived earlier stages) by local circumstances (Prodöhl et al., 2006). The literature review can be considered exploratory.

3. Food enhancement: lobsters and brown crabs are opportunistic in their food preferences. An option to improve populations might be to increase the amount of available food. Potentially low value fish catches that used to be defined as by catch, can serve as additional food supplies. In Sub-WP-E3: Monitoring and habitat modelling, insight will be generated on the food availability in relation to stocks.

\footnotetext{
${ }^{2}$ https://www.nationallobsterhatchery.co.uk/whats-it-all-about/ d.d. 24-05-18, 09-12-18.
} 


\section{$2 \quad$ Literature review on lobster}

This section gives the results of the literature review on European lobster. In its intention it does not aim at being complete on the aspects mentioned. Because the American lobster has a similar size range and general ecology and more literature on this species is available, information on this species is added to the review. Data on $H$. americanus are often used as a model or representative for $H$. gammarus. General feeling is that the species are very much alike and that the data of $H$. americanus can be used as such (Phillips, 2013). Though it should be noted that $H$. americanus is bigger, has migration and occurs to $-700 \mathrm{~m}$, much deeper than $-165 \mathrm{~m}$ of $H$. gammarus (Phillips, 2013). This suggests at least some differences in physiology. When $H$. americanus data are used, it will clearly mentioned.

\subsection{Description}

The scientific name of the European lobster is Homarus gammarus. They possess two large claws, one blunt and designed for crushing and the other sharper and smaller normally used for slicing. Males have larger claws than females, females have wider abdomens (Agnalt et al., 2009, Sørdalen et al., 2018). Their exoskeleton is blue/navy on the dorsal side but slightly lighter underneath with an orange tinge. The body length up to 60 centimetres and weighing up to 5-6 kilograms, although specimen of more than one meter are encountered too. Commercial catches in Cornwall (UK) showed large males of $>150 \mathrm{~mm} \mathrm{CL}$ and females of $140-150 \mathrm{~mm}$ (Hepper, 1978, Figure 6). In Norway up to $140 \mathrm{CL}$ for both males and females. The largest specimen on record measured $1.26 \mathrm{~m}$ and weighed $9.3 \mathrm{~kg}$, caught in 1931 in Fowey, England².

\subsubsection{Age}

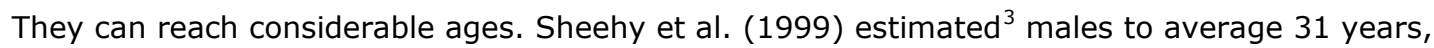
maximum $42 \pm 5$ years and females to average 54 years, maximum $72 \pm 9$ years (Figure 2). Weights were maximally $4.09 \mathrm{~kg}$ for an eleven year old male (166 mm Orbital Carapace Length, OCL, from the back of the eye socket (the orbital), see Appendix 1) and $2.09 \mathrm{~kg}$ for the 72 year old female $(170 \mathrm{~mm}$ $\mathrm{OCL})$. These were all from a commercially bought group in the UK preselected on large size $(>130 \mathrm{~mm}$ $\mathrm{OCL}$, bought at Bridlington and Scarborough considered to have originated primarily from the offshore fishery, 8-20 km from the coast). The sizes of the largest males and females ranged between 150$170 \mathrm{~mm}$ OCL. Appendages and limbs lost through injury can be regenerated, but this usually has a negative effect on both the intermoult period and the growth increment (Jensen et al., 2000, Phillips, 2013).

\subsubsection{Physical characteristics}

Scientifically published data on weight, $\mathrm{CL}$, age, total body length ( $T L$ ) for European lobster are given by Jensen et al. (2000), Kristiansen et al. (2004), Van Stralen and Smeur (2008) Schmalenbach (2009) Schmalenbach et al. (2011), Tangelder et al., 2015) In this study the calculated weight at CL relationships were gathered and given in Table 2 and Figure 3. Observing the graphs the impression is raised that there are differences between locations and differences between male and female lobsters from Helgoland. This could reflect differences in life history (temperature, food conditions, habitat) and sexes. Figure 2 and Figure 4 confirm that males are heavier than females. On the other hand, one should keep in mind that the steep exponential part of the relationship is determined by the larger and heavier animals which are rarely caught. Data points of these larger animals make the graph much

3 It is difficult to estimate age in lobsters. It has to be done indirectly, usually based on the accumulation of lipofuscin. Lipofuscin, is a by-product of cellular metabolism. It accumulates in all cells at a rate determined by metabolic rate of the individual and is quite stable after formation and therefore has met with some success in crustacean age estimation. 
steeper than the equation based on data leaving out these older animals. So differences between these relations can be a mathematical difference rather than an ecological difference.

In Table 2 several measured carapace length at total length (TL) relationships were gathered. This ratio seems rather stable, the slopes are alike. For the relation between $T L$ and $C L$, We calculated a slope of $0.37\left(R^{2}=0.9963\right)$ from the data of Schmalenbach (2009). Kristiansen et al. (2004) calculated a slope of $0.35\left(R^{2}=0,9519\right)$ for reared juvenile European lobsters from Norway, Mercer et al (2001) a slope of 0.36 for reared lobster from Ireland and Scotland.

Table 2. Relations between different physical aspects like carapace length (CL) and weight of different authors and total length (TL) and CL or vice versa for different authors. For Schmalenbach 2009 given year class averages were used to calculate the relations by ourselves

\begin{tabular}{|l|c|c|c|l|}
\hline Origin of animal & Relation & $\mathbf{R}^{\mathbf{2}}$ & Country & Reference \\
\hline CL vs Weight & & & & \\
\hline Reared juveniles & $\mathrm{W}=0.0003 * \mathrm{CL}^{3.1522}$ & 0.9851 & Norway & $\begin{array}{l}\text { Kristiansen et al. } \\
(2004)\end{array}$ \\
\hline $\begin{array}{l}\text { Larger wild lobster caught } \\
\text { in the sea }\end{array}$ & $\mathrm{W}=0.0002 * \mathrm{CL}^{3,2953}$ & 0.9219 & Norway & $\begin{array}{l}\text { Kristiansen et al. } \\
(2004)\end{array}$ \\
\hline $\begin{array}{l}\text { Larger wild lobster caught } \\
\text { in the Oosterschelde }\end{array}$ & $\mathrm{W}=0.000328 * \mathrm{CL}^{3,1708}$ & 0.9779 & Netherlands & $\begin{array}{l}\text { Van Stralen and } \\
\text { Smeur (2008) }\end{array}$ \\
\hline $\begin{array}{l}\text { Larger wild lobster caught } \\
\text { in the Oosterschelde }\end{array}$ & $\mathrm{W}=0.0016 * \mathrm{CL}^{2.803}$ & 0.9840 & Netherlands & $\begin{array}{l}\text { Tangelder et al. } \\
(2015)\end{array}$ \\
\hline $\begin{array}{l}\text { Larger wild female lobster } \\
\text { caught at Helgoland }\end{array}$ & $\mathrm{W}=0.001 * \mathrm{CL}^{2.9211}$ & 0.9996 & Germany & $\begin{array}{l}\text { Schmalenbach } \\
(2009)\end{array}$ \\
\hline $\begin{array}{l}\text { Larger wild male lobster } \\
\text { caught at Helgoland }\end{array}$ & $\mathrm{W}=0.0005^{*} \mathrm{CL}^{3.1046}$ & 0.9982 & Germany & $\begin{array}{l}\text { Schmalenbach } \\
(2009)\end{array}$ \\
\hline $\begin{array}{l}\text { TL vs CL } \\
\text { Wild male and female } \\
\text { lobster caught at } \\
\text { Helgoland }\end{array}$ & $\mathrm{CL}=0.3727 * \mathrm{TL}-5.4775$ & 0.9963 & Germany & $\begin{array}{l}\text { Schmalenbach } \\
(2009)\end{array}$ \\
\hline $\begin{array}{l}\text { Reared juveniles } \\
\text { EBP Juveniles reared in } \\
\text { either Shellfish Research } \\
\text { Laboratory, Carna, or the } \\
\text { Orkney Fisheries } \\
\text { Association in Orkney, } \\
\text { Scotland. }\end{array}$ & $\mathrm{CL}=0.3552 * \mathrm{TL}+0.170$ & 0.9519 & Norway & $\begin{array}{l}\text { Kristiansen et al. } \\
(2004)\end{array}$ \\
\hline
\end{tabular}




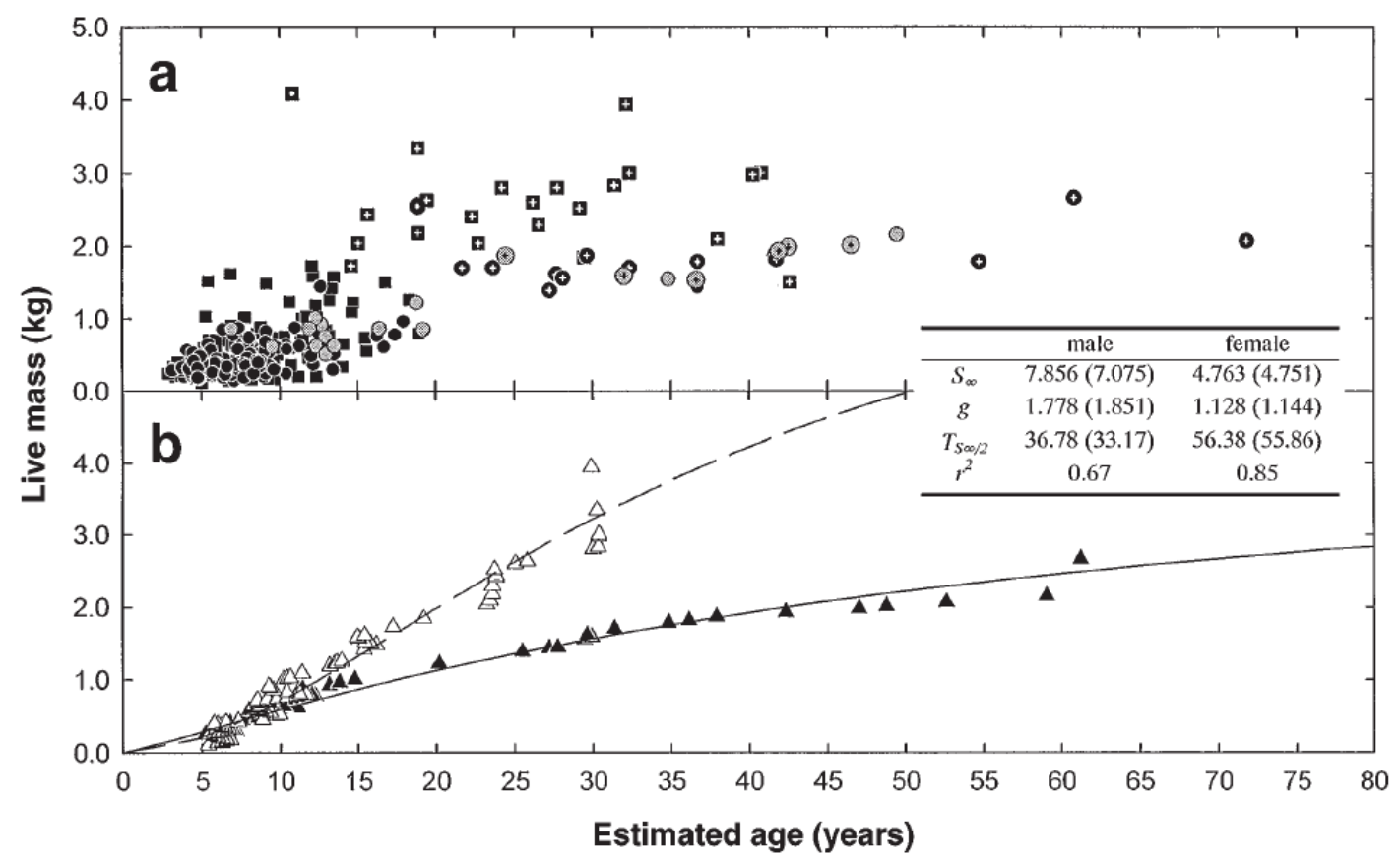

Figure 2. Relationship between live mass and estimated age for sampled wild Yorkshire H. gammarus. (a) Raw estimated age - live mass data: squares, males; circles females; solid black symbols, Yorkshire lobsters collected in 1996 and originating primarily from inshore fishing grounds with gravid females shown as solid grey circles; black symbols with cross, Yorkshire lobsters collected in 1997 and originating primarily from offshore fishing grounds with gravid females shown as grey circles with cross; black symbols with dot, Cornish lobsters. Live mass data were not available for the reference lobsters.

(b) LOWESS-smoothed data from Figure 2a, excluding Cornish lobsters: open triangles, males; black triangles, females; dashed and solid lines, best-fitting logistic models for males and females, respectively. The regression coefficients for the models are shown in the inset. Values in parentheses and $r^{2}$ coefficients refer to the same model when fitted directly to the raw data in Figure 2a. Figure taken from Sheehy et al. (1999). 


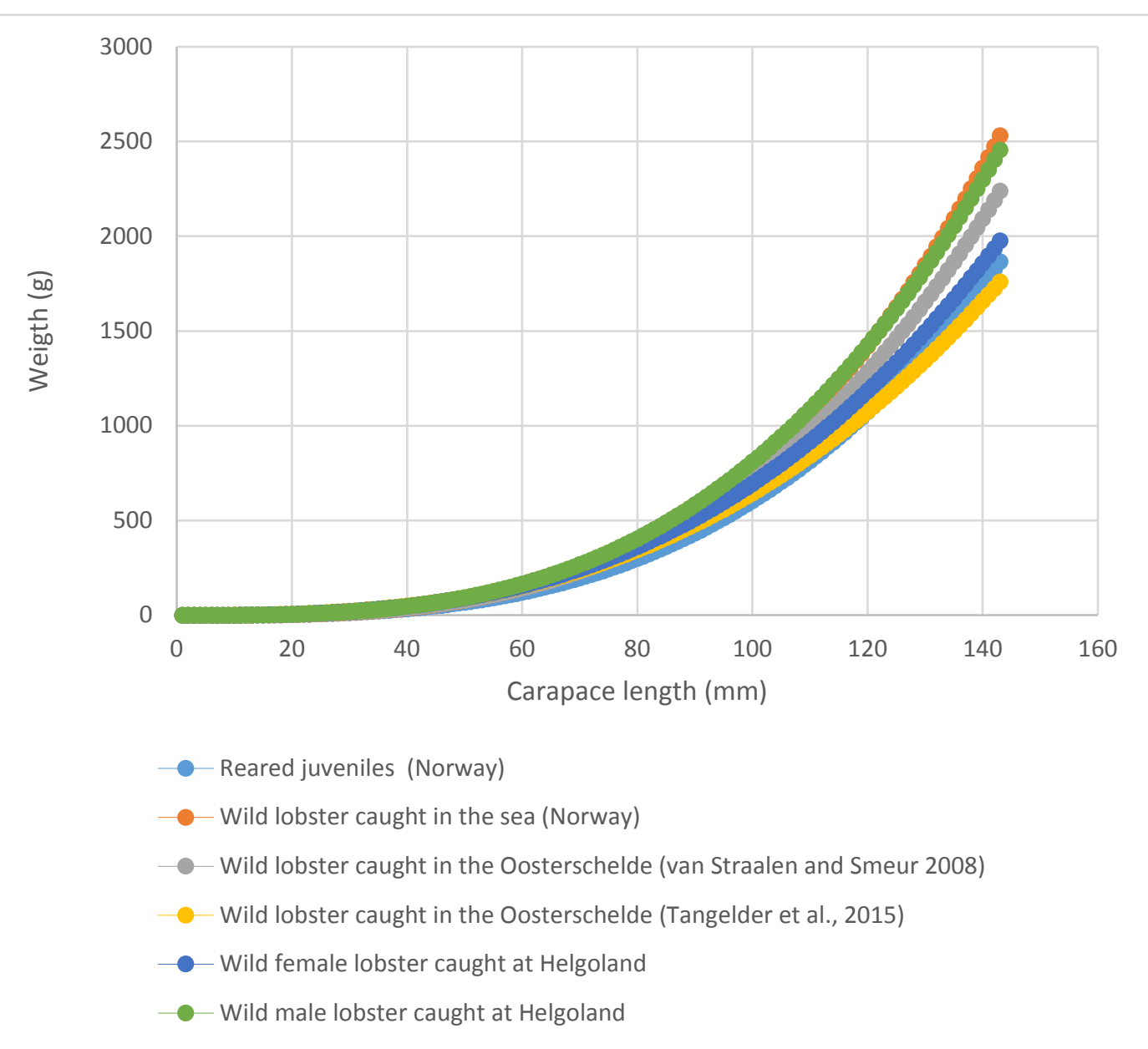

Figure 3. Calculated weight at carapace length relationships obtained from Kristiansen et al., 2002, van Stralen and Smeur, 2008, Schmalenbach (2009), Tangelder et al. (2015). For the weight at carapace length relationship of Schmalenbach (2009) the average data for each year class were used.
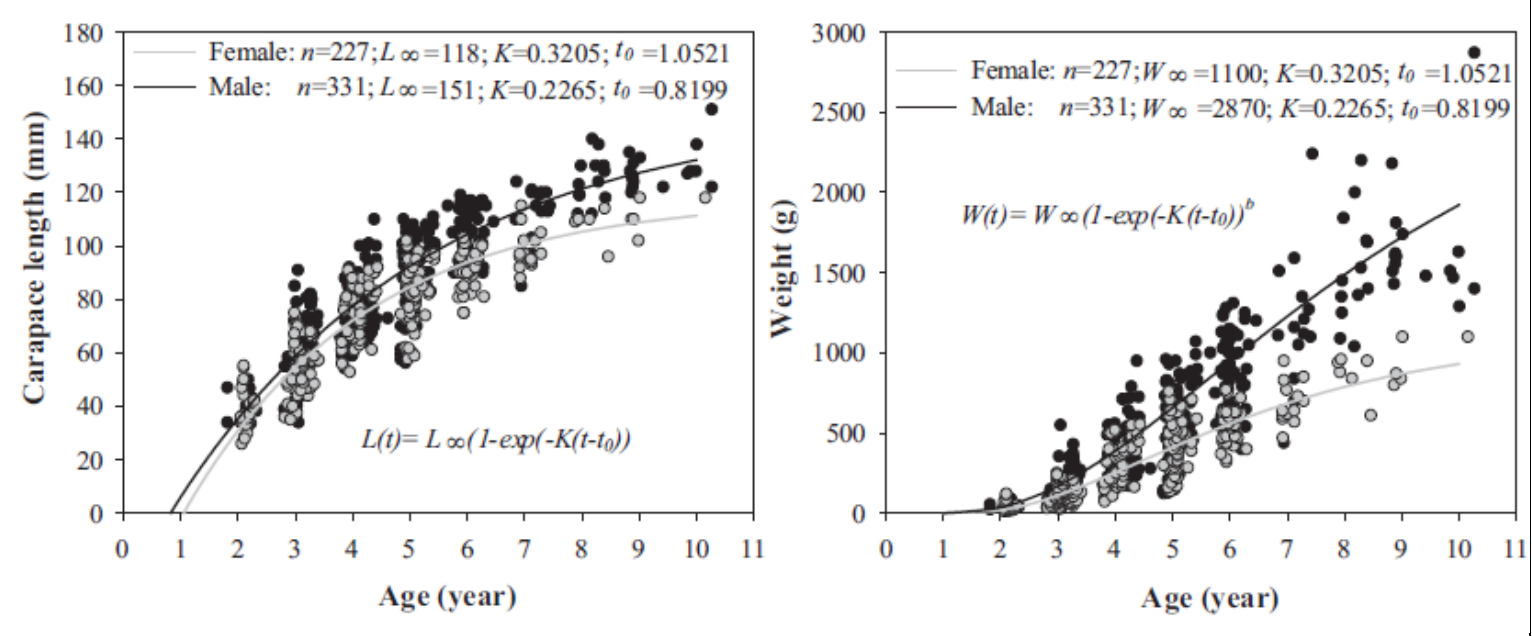

Figure 4. Growth curves from length-at-age and weight-at-age data for female and male European lobsters (Homarus gammarus) from Helgoland (Germany) using the von Bertalanffy growth function (Schmallenbach et al., 2011) 


\subsection{Distribution}

The European lobster is distributed throughout coastal areas of the northeast Atlantic from midNorway to the North Sea, south to the north African coast and into the eastern Mediterranean (Figure 7, Phillips, 2013). However, their 'commercial range' is currently limited to northern France, Britain and Ireland. The northernmost populations are found in the Norwegian fjords Tysfjorden and Nordfolda, inside the Arctic Circle (Agnalt et al., 2009). In Norway and Shetland stocks have declined significantly or collapsed (Figure 5). In the Mediterranean and off the Iberian peninsula, north to the Bay of Biscay, lobsters are rare and generally well below commercial levels. Their stronghold, therefore, is a relatively small proportion of their previous commercial distribution (Holthuis 1991, Prodöhl et al., 2006, Phillips, 2013).

\subsection{Landings}

\subsubsection{Regulations and management on landings}

European regulations on European lobster state (European Commission, 2006): "The catching, keeping on board, transhipping, landing, storing, selling and displaying or offering for sale of berried female crawfish (Palinuridae spp.) and berried female lobster (Homarus gammarus) shall be prohibited. Berried female crawfish and berried female lobster shall be returned to sea immediately after accidental catching or may be used for direct restocking and transplantation within management plans established pursuant to either Articles 18 or 19 of this Regulation."

Dutch regulations state that with all gears egg-bearing lobsters, freshly caught lobsters and lobsters caught between July 15 and the last Thursday of March are put back immediately after the catch ${ }^{4}$.

The minimum conservation reference size (MCRS) in Dutch Belgium and German waters $85 \mathrm{~mm} \mathrm{CL}^{5}$ (Schmalenbach, 2009); in British waters is $87 \mathrm{~mm}^{6}$; in Norwegian waters 88mm (Agnalt et al., 2009). In 2019, parts of the West and North coasts of Scotland and south west England (Cornwall) will have a MCRS of $90 \mathrm{~mm} \mathrm{CL}$ and the East coast remains $87 \mathrm{~mm}^{2,7}$. The American lobster Homarus americanus has a MCRS of $83 \mathrm{~mm} \mathrm{CL}$ in Maine (Dunnington et al., 2010).

Also V-noticing is applied on female tails after which they are returned ensuring at least one possibility to bear eggs.

\subsubsection{Landings}

In the past 70 years, the total annual European landings varied between 1,600 and 5,600 tonnes (Figure 5, Prodöhl et al., 2006, Phillips, 2013). At the beginning of the sixties an annual catch of 3,000 $-3,500$ tons was not uncommon, but the annual catch dropped in the 1970 s to less than 2,000 tons at the beginning of the eighties. Since then, a slow increase to 3,200 tons has been observed and after 2006 to 2010 a revival to about 5,000 tons (Phillips, 2013). Lobster catches vary considerably from country to country: between 1950 and 1975, Scotland accounted for 26\% of total landings; Norway for $18 \%$, followed by England, Wales and France with $16 \%$, Ireland with $9 \%$ and Sweden, Denmark and Spain with less than 5\%. Before the 1960s, Norway recorded annual catches of 600 to 1,000 tonnes, but during the following two decades a collapse of fishing was observed and annual catches are now less than 60 tonnes (Figure 5). Within the Mediterranean countries, the annually reported landings have never reached the same levels as those in the north-western distribution area (Prodöhl et al., 2006). In the year 2016 the Netherlands landed 64 tonnes (1.4\%); the UK 3281 (71\%); France

\footnotetext{
https://wetten.overheid.nl/BWBR0024539/2017-10-05 d.d. 20-12-18

http://www.zeevissers.com/minimummatenzeevis.html D.d. 07-12-18.

https://www.gov.uk/government/publications/minimum-conservation-reference-sizes-mcrs/minimum-conservationreference-sizes-mcrs-in-uk-waters\#crustaceans d.d. 07-12-18,

7 https://www2.gov.scot/Topics/marine/Sea-Fisheries/InshoreFisheries/crab-lobster-landingcontrols?fbclid=IwAR2Qrk19mUois5g9wyKiO8V5SYRpUaWHTqFpNKLigFWhW6e2we0LcL9Lf78
} 
587 tonnes (13\%); Ireland 138 tonnes (3\%) and Norway 54 tonnes (1.2\%), summing with other countries altogether to 4656 tonnes landed in 2016.

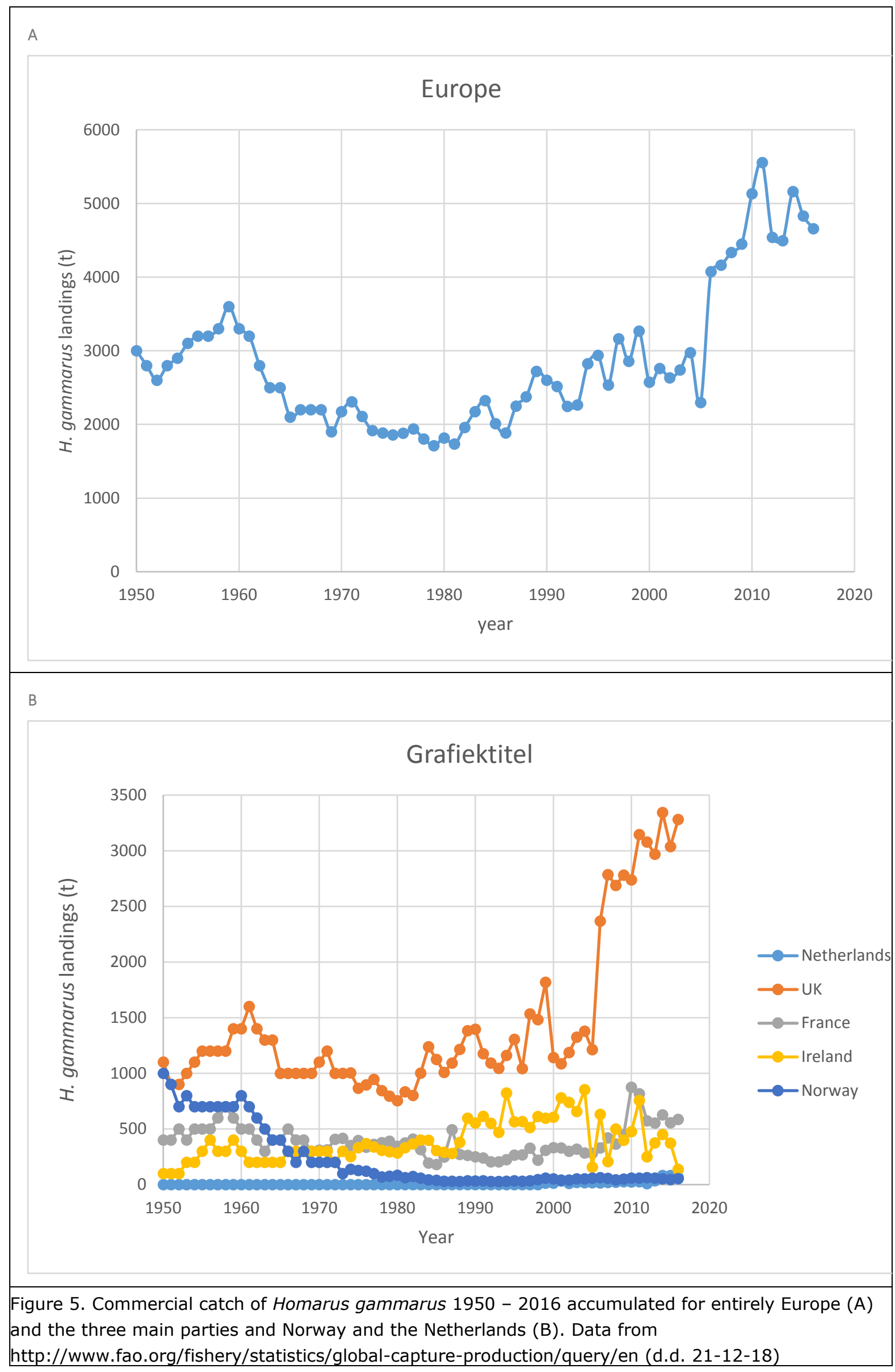




\subsubsection{Sizes caught}

Giving some examples without trying to be extensive, the size distributions of male and female European lobsters caught on the Northumberland coast (North sea, UK) were around $\sim 80-82 \mathrm{~mm} \mathrm{CL}$ (Skerrit at al., 2012). Average sizes estimated were e.g. 83 to $88 \mathrm{~mm} \mathrm{CL}$ in Cornwall and $77 \mathrm{~mm} \mathrm{CL}$ in Yorkshire (Hepper, 1978), in Westermost Rough Offshore Windfarm Park (near the Humber, North sea, UK) most abundant class of lobsters after a period of closure for fisheries was $~ 87 \mathrm{~mm} \mathrm{CL}$ class whereas the control site had the most abundant size class at $\sim 80 \mathrm{~mm} \mathrm{CL}$ (Roach et al., 2018). In Norway average sizes were encountered between $74 \mathrm{~mm}$ (Stefjord, a special genetic subpopulation) and $108 \mathrm{~mm} \mathrm{CL}$ (Stavfjord) in Norway (Agnalt et al., 2009). The lobsters caught in lobster pots are usually 23-38 cm long and weigh 0.7-2.2 kg. Hepper (1978) showed 75-90 mm CL range being the most frequent commercially caught lengths in Cornwal (Figure 6). Schmalenbach et al. (2011) caught larger European lobsters at Helgoland with the main fraction between 90 to $130 \mathrm{~mm} \mathrm{CL}$.
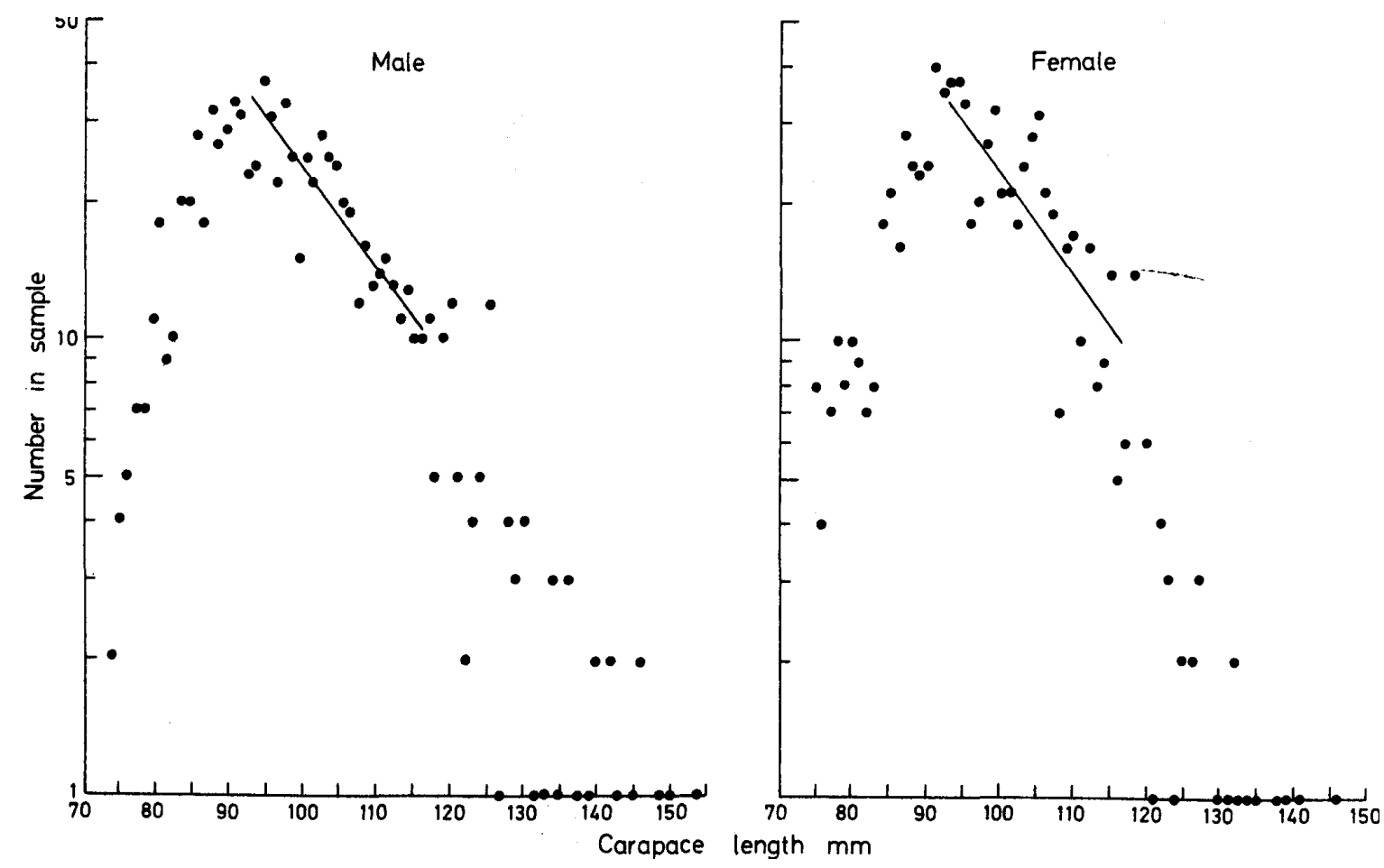

Figure 6. Size frequency distribution of lobsters measured from the Cornwal lobster fishery plotted on a logarithmic scale (the catch curve) (Hepper, 1978).

\subsection{Biology and habitat}

European lobsters are usually located at lower than mean low water neaps (sublittoral fringe) to depths of $165 \mathrm{~m}$; usually not deeper than $50 \mathrm{~m}$ (Table 3). The LD 50 for adult $H$. gammarus was at a salinity of $24 \%$ according to Mercer at el. (2001, temperature ranging from 9 to $11^{\circ} \mathrm{C}$ ) and according Wolf and Sandee (1971) lobsters from the Oosterschelde and Veerse Meer can survive down to $9 \%$ during wintertime $\left(\sim 3^{\circ} \mathrm{C}\right)$. EBP stage European lobsters had a LD50 of $16 \%$ o (Mercer et al., 2001$)$. Low salinities will lead to reduced moulding and below $11 \%$ no more moulding will occur (Schuiling and Smaal, 1998). Older and larger lobster are encountered in deeper regions, although this could be an impact of catchment rather than habitat preference. They are vulnerable to current speeds of 0.6 $\mathrm{m} / \mathrm{s}$ and higher and wave energy. Then they get carried away (Howard, 1988, Linely et al., 2008, van den Boogaard et al., 2019). 
They are usually found on hard substrates: rock or hard mud, but also soft substrates are used for food and nursery grounds. The animals are nocturnal and territorial, living in holes or crevices (Holthuis, 1991, Lawton \& Lavalli, 1995, Jensen et al., 2000, Turner et al., 2009, Phillips, 2013, Seitz et al., 2014, Skerrit et al., 2015). Holes and crevices are especially important for the younger stages that are vulnerable to predation and cannibalism (Table 4, Jensen et al., 2000, Mercer et al., 2001, Phillips, 2013). As lobsters grow they gain a size-refuge from predators, and in turn their association with shelter tends to relax. Therefore, smaller lobsters are more common in the middle of cobble patches, whereas large lobsters are more common on edges (Skerrit, 2014). In England, areas with habitats that include less structure and fewer large-scale outcrops for adults produce lobsters of smaller size than other areas, indicating the importance of the habitat for growth (Jensen et al., 1994). Crevices and ledges remain still important (see e.g. Howard, 1988, Jensen et al., 2000, Galparsoro et al., 2009). Table 4 (taken from Lawton \& Lavalli, 1995) gives an excellent overview of the different life history phases of $H$. americanus with associated behaviour and ecological processes, which might be quiet valid for $H$. gammarus as well (Jensen et al., 2000, Phillips, 2013). Note that the sizes of the different stages are slightly different. And the column on realms occupied emphasis the seasonal movement of $H$. americanus, which is not applicable to $H$. gammarus, which remain mostly at one location once having encountered a suitable crevice.

Like other crustaceans, lobsters have a hard exoskeleton which they must shed in order to grow, in a process called ecdysis (moulting). This may occur several times a year for young lobsters, but decreases to once every 1-2 years for larger animals until becoming an annual part of the mating, spawning and egg hatching cycle (Hepper, 1978, Holthuis, 1991, Phillips, 2013).

Pre-adult and adult lobsters are reef-obligate and select sites that supply sufficient food and oxygen and shelter from currents and predation (Linley et al., 2008, Table 4). A number of criteria, including oxygen supply, length, entrance size, presence of multi-openings (escape routes), internal aspect ratio (manoeuvring space) are all aspects that may influence the suitability of cracks and crevices for lobsters (Howard, 1988, Lawton \& Lavalli, 1995, Jensen et al., 1994, 2000, Linley et al., 2008). As lobsters grow (in steps following ecdysis) they need to move to increasingly larger crevices. As a given cohort of lobsters grows, it suffers mortality that is dependent on a number of factors. These include predation, competition and diseases.

Table 3 gives rather broad ranges for the tolerance of European and American lobsters towards abiotic factors $^{8}$. European Lobsters have a large range of distribution (Figure 7) expressing both this tolerance and the suitability of local habitat. In general it is thought that suboptimal circumstances (in salinity, oxygen etc.) will lead to higher metabolic costs and reduced growth (Jensen et al., 2000, Linley et al., 2008). There estuarine environments, with altering salinities and particularly those associated with periodic reductions in temperature, these conditions are stressful these conditions are stressful and likely to be predominantly unsuitable environments

A first wreck suitability map for the Dutch NCP is made by Van den Boogaart et al. (2019, Figure 8) based on divers observations on wrecks. Suitable and less suitable spots appear next to each other whereas abiotic circumstances seem not that differentiating (Table 7, Smaal et al., 2017). More research is needed in order to determine habitat suitability and differentiating factors on the Dutch NCP.

$8 \mathrm{Nb}$ data on $\mathrm{H}$. americanus are often used as a model or representative for $\mathrm{H}$. gammarus. General feeling is that the species are very much alike and that the data of $\mathrm{H}$. americanus can be used as such (Phillips, 2013). Though it should be noted that $H$. americanus is bigger, has migration and occurs to $-700 \mathrm{~m}$, much deeper than $-165 \mathrm{~m}$ of $H$. gammarus (Phillips, 2013). This suggests at least some differences in physiology. 


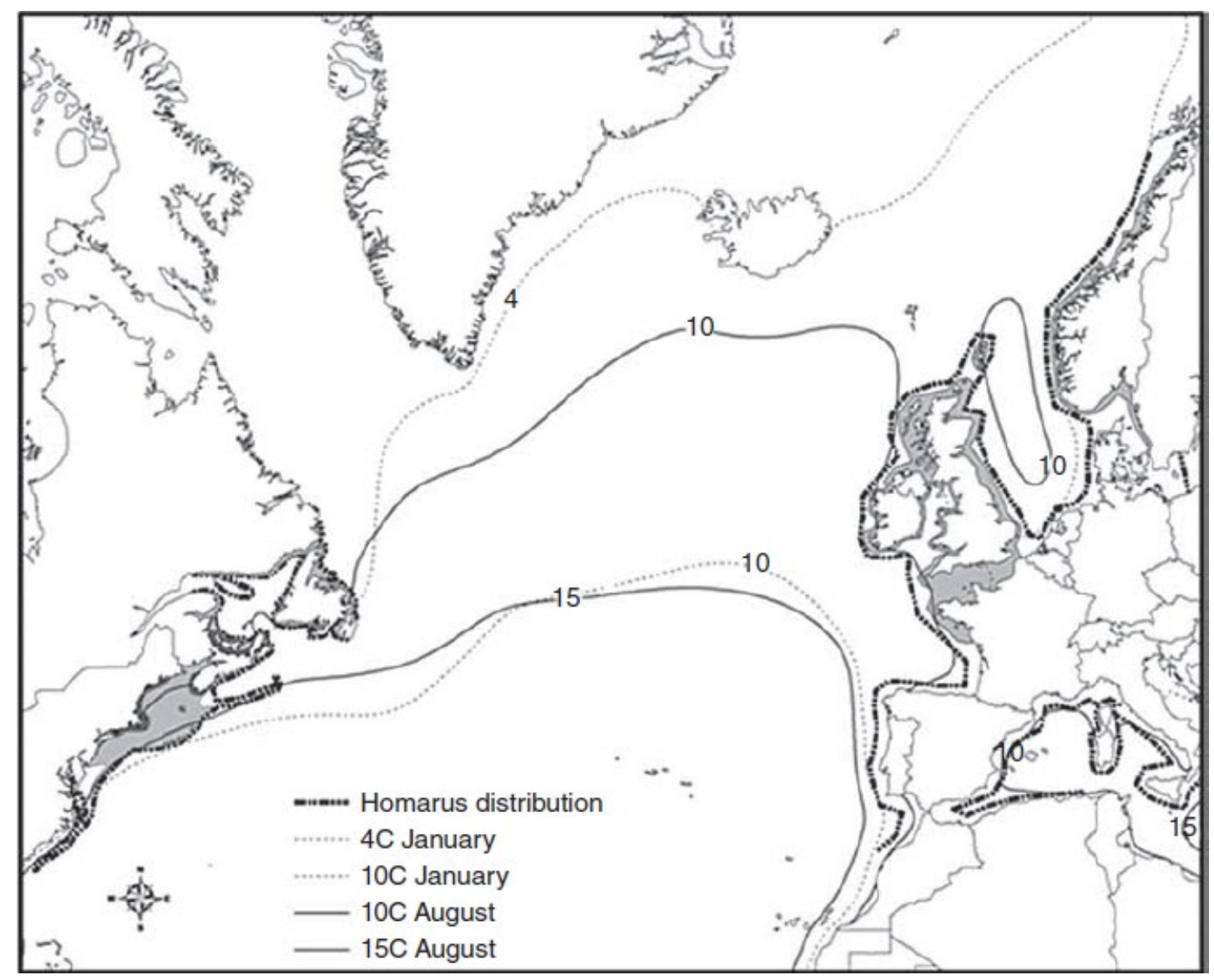

Figure 7 Geographic distribution of $H$. americanus and $H$. gammarus in the North Atlantic relative to seasonal isotherms. Dotted boundary is species geographic range; areas in grey are prime commercial fishing grounds (from Phillips, 2013).

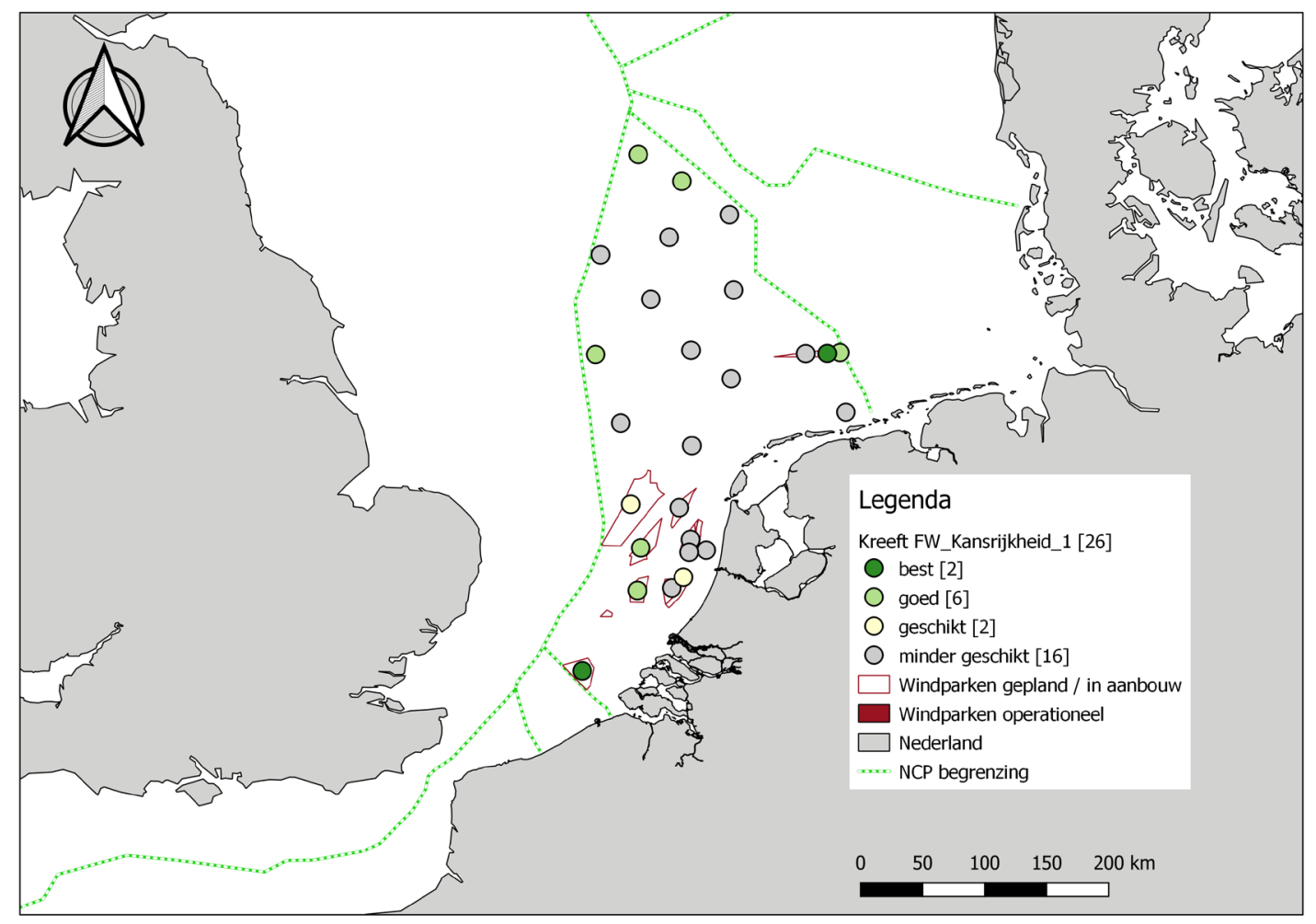

Figure 8 Opportunity map for catching North Sea lobster H. gammarus at (potential) offshore wind energy locations for the Dutch NCP (van den Bogaart et al., 2019). 
Table 3. Ranges of relevant abiotic aspects under which $H$. gammarus is encountered.

\begin{tabular}{|c|c|c|c|c|c|}
\hline \multicolumn{4}{|c|}{$\begin{array}{l}\text { Desirable levels for key water quality parameters for Homarus sp. (van Olst et } \\
\text { al. } 1980 \text { (H. americanus) and Wickins \& Lee } 2002,(H \text {. gammarus)), Table } \\
\text { compiled by Kristiansen et al. (2004). Data from Oosterschelde lobster from } \\
\text { Wolf and Sandee (1971) and Schuiling and Smaal (1998). The bold figure here } \\
\text { is claimed to be the optimum }\end{array}$} & \multicolumn{2}{|c|}{$\begin{array}{l}\text { Natural ranges in which } H . \text { gammarus is encountered } \\
\text { (https://eol.org/pages/46505673_d.d_10-12-18) }\end{array}$} \\
\hline Parameter & $\begin{array}{c}\text { Optimal } \\
\text { condition }\end{array}$ & $\begin{array}{c}\text { Natural } \\
\text { range }\end{array}$ & Lethal condition & & Encountered range \\
\hline Temperature $\left({ }^{\circ} \mathrm{C}\right)$ & $18-22$ & $1-25$ & $<0,>31$ & $\begin{array}{l}\text { Temperature range } \\
\left({ }^{\circ} \mathrm{C}\right)\end{array}$ & $8.214-12.243$ \\
\hline Salinity (\%o) & $28-35$ & $28-35$ & $<8,>45$ & Salinity (\%o) & $33.5-35.6$ \\
\hline $\begin{array}{l}\text { Salinity (\%o) } \\
\text { lobsters } \\
\text { Oosterschelde }\end{array}$ & $21-27-32$ & $9-35$ & & & \\
\hline $\mathrm{O} 2\left(\mathrm{mg} \mathrm{l}^{-1}\right)$ & 6.4 & $4-8.2$ & $<1$, $>$ saturation & Oxygen $\left(\mathrm{mg} \mathrm{l}^{-1}\right)$ & $5.4-6.7$ \\
\hline $\mathrm{pH}$ & 8 & $7.8-8.2$ & $<5,>9$ & & \\
\hline Ammonia $\left(\mathrm{mg} \mathrm{l}^{-1}\right)$ & $<0.14$ & $0-0.3$ & $>1.4$ & & \\
\hline & & & & Depth range $(\mathrm{m})$ & $0-165$ \\
\hline & & & & Nitrate $\left(\mu \mathrm{mol} \mathrm{l} \mathrm{l}^{-1}\right)$ & $2.7-8.5$ \\
\hline & & & & Phosphate $(\mu \mathrm{mol} \mathrm{l-1})$ & $0.32-0.63$ \\
\hline & & & & Silicate $\left(\mu \mathrm{mol} \mathrm{I} \mathrm{I}^{-1}\right)$ & $1.9-4.5$ \\
\hline
\end{tabular}


Table 4. Life history phases of Homarus americanus with associated behaviour and ecological processes (from Lawton \& Lavalli, 1995). Sizes at transition between all phases may vary geographically. For adolescent and adult lobsters, the sizes specified are the minimum carapace lengths (CLs) for entry into these life history phases. Size at maturity varies geographically, such that functional maturity

\begin{tabular}{|c|c|c|c|c|c|c|}
\hline Phase & $\begin{array}{l}\text { Size (mm } \\
\quad \text { CL) }\end{array}$ & Activity pattern & $\begin{array}{l}\text { Foraging } \\
\text { mode }\end{array}$ & $\begin{array}{l}\text { No. of shelters } \\
\text { occupied }\end{array}$ & $\begin{array}{l}\text { Realms (maximum } \\
\text { abundance) }\end{array}$ & Ecological processes \\
\hline $\begin{array}{l}\text { Larval } \\
\text { (stages I- } \\
\text { III) }\end{array}$ & $\sim 2-4$ & $\begin{array}{l}\text { Vertical migrator; } \\
\text { poor swimmer; } \\
\text { passive (?) drifter }\end{array}$ & $\begin{array}{l}\text { Raptorial } \\
\text { feeder }\end{array}$ & NIA & $\begin{array}{l}\text { Pelagic (offshore to } \\
\text { inshore depending on } \\
\text { area) }\end{array}$ & $\begin{array}{l}\text { Dispersal; } \\
\text { development }\end{array}$ \\
\hline $\begin{array}{l}\text { Postlarval } \\
\text { (stage IV) }\end{array}$ & $\sim 4-5$ & $\begin{array}{l}\text { Strong surface swimmer; } \\
\text { benthic settler }\end{array}$ & $\begin{array}{l}\text { Raptorial/ } \\
\text { suspension } \\
\text { feeder }\end{array}$ & $\begin{array}{l}\text { Selects preexisting } \\
\text { shelter or } \\
\text { excavates new one }\end{array}$ & Pelagic(inshore) to benthic & $\begin{array}{l}\text { Settlement; predation } \\
\text { while settling or shortly } \\
\text { thereafter }\end{array}$ \\
\hline $\begin{array}{l}\text { Shelter- } \\
\text { restricted } \\
\text { juvenile }\end{array}$ & $\sim 4-14$ & $\begin{array}{l}\text { Recent settler; remains } \\
\text { under cover; subterranean } \\
\text { movements within spatially } \\
\text { complex habitats }\end{array}$ & $\begin{array}{l}\text { Suspension } \\
\text { feeder; } \\
\text { browser } \\
\text { within } \\
\text { shelter; } \\
\text { ambusher at } \\
\text { shelter } \\
\text { entrance }\end{array}$ & $\begin{array}{l}\text { Usually one, } \\
\text { sometimes several } \\
\text { within a contiguous } \\
\text { shelter space }\end{array}$ & Benthic (shallow) & Recruitment; predation \\
\hline $\begin{array}{l}\text { Emergent } \\
\text { juvenile }\end{array}$ & $\sim 15-25$ & $\begin{array}{l}\text { Mostly shelter confined; } \\
\text { limited movements } \\
\text { outside of shelter, but } \\
\text { remains in close vicinity }\end{array}$ & $\begin{array}{l}\text { Browser; } \\
\text { ambusher }\end{array}$ & $\begin{array}{l}\text { One to } \\
\text { several }\end{array}$ & Benthic (shallow) & Predation \\
\hline $\begin{array}{l}\text { Vagile } \\
\text { juvenile }\end{array}$ & $\begin{array}{l}\sim 25-\text { size } \\
\text { of } \\
\text { physiological } \\
\text { maturity } \\
(\sim 40)\end{array}$ & $\begin{array}{l}\text { Shelter user, but more } \\
\text { extensive movements out of } \\
\text { shelter for food }\end{array}$ & $\begin{array}{l}\text { Ambusher; } \\
\text { pursuer; } \\
\text { searcher }\end{array}$ & One to several & Benthic(shallow) & Competition \\
\hline Adolescent & $\begin{array}{l}\text { Physiological } \\
\text { maturity, } \\
\text { but not } \\
\text { functional } \\
\text { maturity } \\
(\sim 50) \\
\end{array}$ & $\begin{array}{l}\text { Active, mostly nocturnal; } \\
\text { may participate in } \\
\text { seasonal movements } \\
\text { with reproductive } \\
\text { animals }\end{array}$ & $\begin{array}{l}\text { Pursuer; } \\
\text { searcher }\end{array}$ & $\begin{array}{l}\text { One to many, } \\
\text { depending on } \\
\text { seasonal } \\
\text { movement }\end{array}$ & Benthic (shallow to deep) & $\begin{array}{l}\text { Competition; indirect } \\
\text { fishing mortality due to } \\
\text { retention in traps }\end{array}$ \\
\hline Adult & $\begin{array}{l}\text { Functional } \\
\text { maturity } \\
(>50)\end{array}$ & $\begin{array}{l}\text { Active, mostly nocturnal; } \\
\text { seasonal, reproductively } \\
\text { mediated movements }\end{array}$ & $\begin{array}{l}\text { Pursuer; } \\
\text { searcher }\end{array}$ & $\begin{array}{l}\text { One to many, } \\
\text { depending on } \\
\text { seasonal } \\
\text { movement }\end{array}$ & Benthic (shallow to deep) & $\begin{array}{l}\text { Mate selection; } \\
\text { reproductive success; } \\
\text { direct fishing mortality }\end{array}$ \\
\hline
\end{tabular}



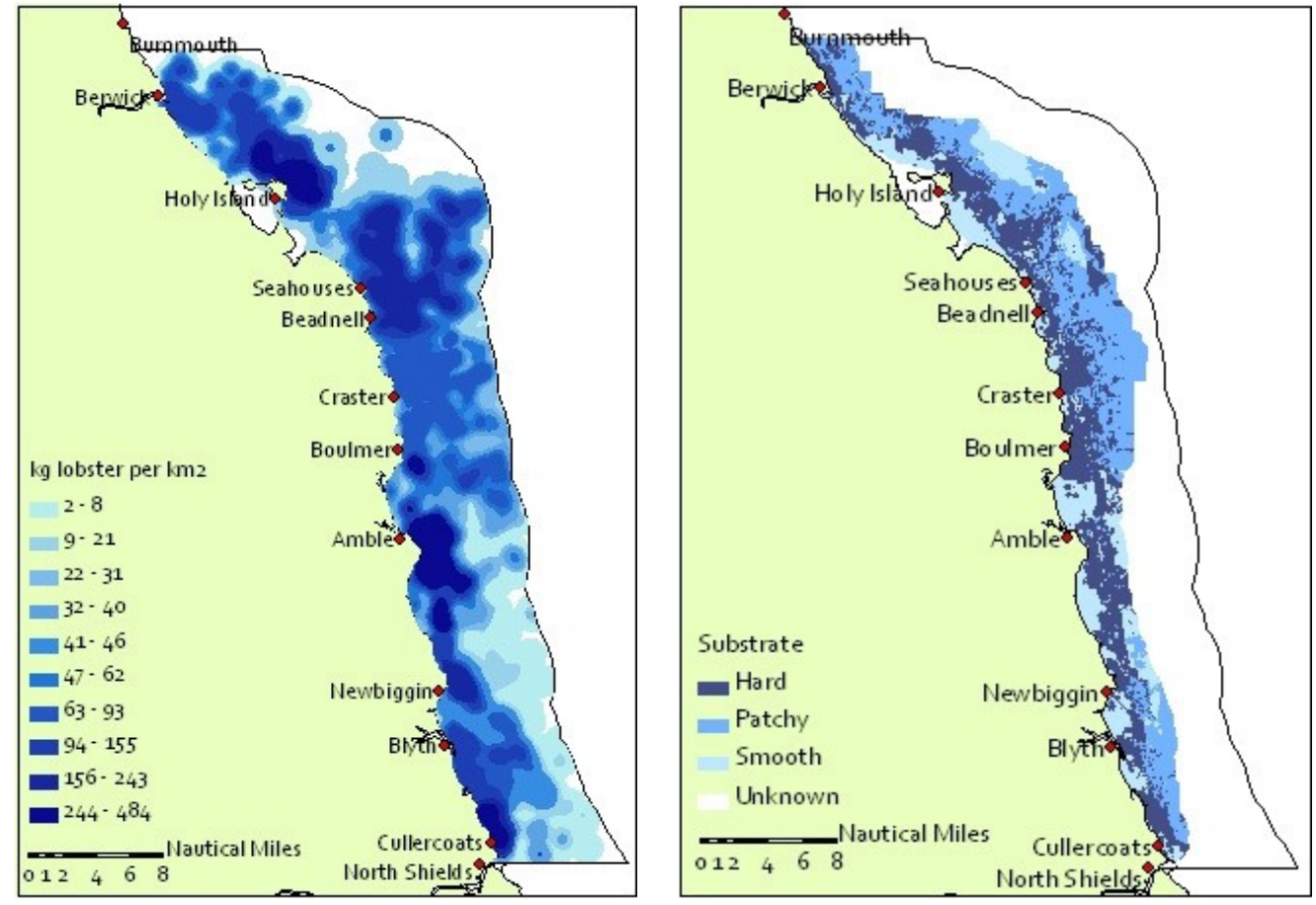

Figure 9. data from Northumberland, UK (from Turner et al., 2009).

A. Estimated distribution of lobster landings by port (mean annual totals 2004-2007)

B. Larger scale substrate characterisation near Blyth

\subsection{Densities}

In general, density of the European lobster is irregular and seems to be regulated by the presence of hard substrate however not always (Figure 9, Figure 10, Figure 13, Turner et al., 2009, Skerrit et al., 2012, 2015, Wallace, 2015, Wright, 2018). In this sense it is important to define what is considered the seize of the area of reference and the resulting density.

An important finding is that there is little density data in literature on $H$. gammarus. There is a clear omission in this sense. We will give an overview of what was found on $H$. gammarus and also provide some densities of $\mathrm{H}$. americanus (see also Table 5). Agnalt et al. (2009) estimated average density of lobsters in $\mathrm{km}^{-1}$ shoreline, not in $\mathrm{km}^{-2}: 169$ ( \pm 79) lobsters km-1 shoreline in Stefjord, $189( \pm 122)$ lobsters km-1 in Mannfjord, and 99 ( \pm 39) lobsters km-1 in Mørsvikfjord. Turner et al. (2009) estimated landings of $\mathrm{kg}$ lobsters on Northumberland, UK, giving some indications for densities (Figure 9, Figure 10). They estimated landings per unit area from a minimum of $2 \mathrm{~kg} \mathrm{~km}^{-2} \mathrm{yr}^{-1}$, to a maximum of 484 $\mathrm{kg} \mathrm{km}^{-2} \mathrm{yr}^{-1}$ (at an average weight of $0.5 \mathrm{~kg}$ per lobster is $\sim 0.001$ lobster caught $\mathrm{m}^{-2}$ ). The highest estimates of landings per unit area were at Holy Island, with maximum values at all other ports below $300 \mathrm{~kg} \mathrm{~km}^{-2} \mathrm{yr}^{-1}\left(\sim 0.0006\right.$ lobster $\left.\mathrm{m}^{-2} \mathrm{yr}^{-1}\right)$.

In literature densities are encountered ranging from 0.00037 calculated with the entire surface of the Oosterschelde or (van Stralen and Smeur, 2008), 0.002 (Skerrit, 2014, Skerrit et al., 2012) to 0.27 lobsters $\mathrm{m}^{-2}$ (Jensen et al., 1994) (see Table 5). The density given by Jensen et al., (1994) is high. It represents a situation with an artificial reef with high densities of crevices especially for lobsters. In Blyth a summer/winter difference was encountered in densities (Skerrit, 2014, Skerrit et al., 2012, Table 5, Figure 10). In winter a density of 0.002 lobsters $\mathrm{m}^{-2}$ and in summer 0.006 to 0.007 lobsters $\mathrm{m}^{-2}$. Jensen et al. (2000) and Howard (1988) give overviews on densities in which they cited personal communications ranging from around 0.03 lobsters $\mathrm{m}^{-2}$ (unspecified British sites; R.J. Handford cited in Howard, 1988) similar to observations in Lough Hyne of 0.03 lobsters $\mathrm{m}^{-2}$ (Robinson et al., in preparation, in Jensen et al., 2000, based on observed nearest neighbour distances ranged from 0.75 
$\mathrm{m}$ to $35 \mathrm{~m}$ ). In a natural, horizontal rock crack in Scottish waters, densities were encountered from 0.4 to $5.0 \mathrm{~m}^{-2}$ (mean $1.5 \mathrm{~m}^{-2}$ Comelyand Ansell, personal communication, cited by Jensen et al., 2000). Van Stralen and Smeur (2008) estimated a total of 130000 lobsters in the Oosterschelde. We calculated that to a roughly estimated 0.00037 lobsters $\mathrm{m}^{-2}$ when using the entire surface of the Oosterschelde $\left(350 \mathrm{~km}^{2}\right)^{9}$. Using an estimated dyke surface (240 km total length according to Brummelhuis et al. (2013)) and an average of $9 \mathrm{~m}$ depth ${ }^{10}$, the roughly estimated densities are much higher: 0.060 lobster $/ \mathrm{m}^{2}$. It should be noted average depth takes also the shallow parts (like schoals and mud flats) of the Oosterschelde into account whereas most dykes are next to the deep tidal channels, reducing the density due to a larger average depth. In addition in the approach the dyke is a vertical wall whereas in fact it is an slowly sloping surface, also increasing the surface of the dykes thereby reducing the average. A tenth of the estimated density seems more appropriate (triple average depth and triple length of the slope). The densities on Dutch NCP soft sediments were roughly estimated at 0.000002 larger lobsters $\mathrm{m}^{-2}$ (2 lobsters $\mathrm{km}^{-2}, \mathrm{P}$. Molenaar, WMR, pers. comm.).

For $H$. americanus densities were encountered up to 0.340 lobsters $\mathrm{m}^{-2}$ (Table 5 ). Given the migration behaviour of $H$. americanus densities vary in time and also depend on the substrate (Dunnington et al., 2005, Geraldi et al., 2009, Phillips, 2013).

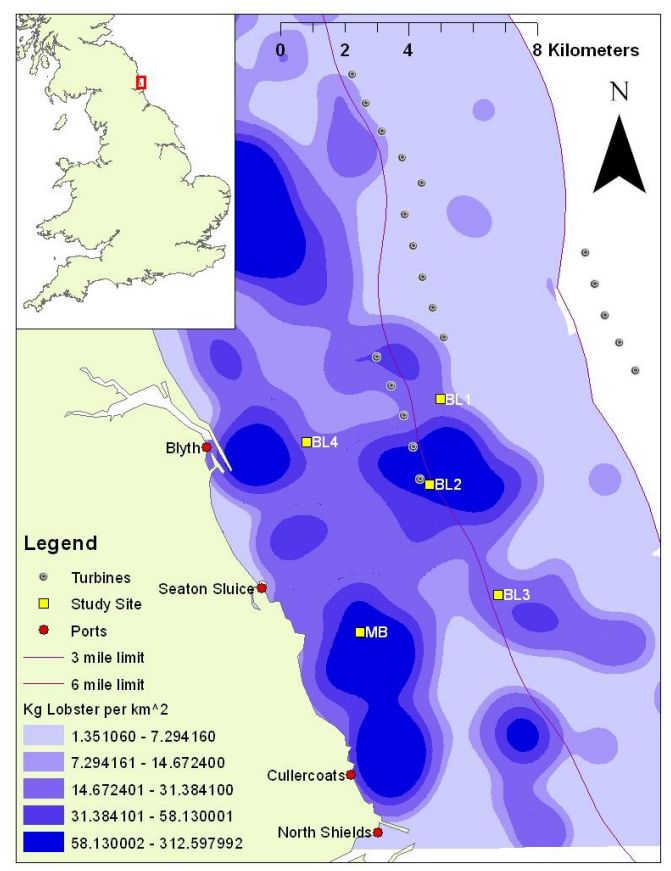

Figure 10. Lobster distribution near Blyth, Northumberland, UK based on landings, vessel sightings and average vessel home-range (from Skerrit et al., 2012). The spot B4 is where Skerrit et al (2012, 2015, ) and Skerrit (2014) have performed their research. Figure 13 gives more details of location B4.

\footnotetext{
${ }^{9}$ https://nl.wikipedia.org/wiki/Nationaal_Park_Oosterschelde d.d. 26-03-19.

${ }^{10} \mathrm{https}: / /$ duikeninbeeld.tv/scubapedia-oosterschelde/ d.d. 26-03-19

22 of 64 | Wageningen Marine Research report C109/18
} 
Table 5. Different densities at which $H$. gammarus (EL) and $H$. americanus (AL) are encountered. Only traceable densities published in journals or grey literature are used.

\begin{tabular}{|c|c|c|c|c|c|c|c|c|c|c|c|c|}
\hline Species & \begin{tabular}{|c|} 
Average \\
density \\
(ind/m2)
\end{tabular} & $\begin{array}{l}\text { Average } \\
\text { m2 per } \\
\text { individual } \\
\text { ( } \mathrm{m} 2 / \text { ind })\end{array}$ & $\begin{array}{l}\text { Size class } \\
\text { average } \\
\text { CL }(\mathrm{mm})\end{array}$ & $\begin{array}{l}\text { Size } \\
\text { range } \\
\text { Low } \\
(\mathrm{mm})\end{array}$ & \begin{tabular}{|c|} 
Size \\
range \\
High \\
$(\mathrm{mm})$
\end{tabular} & Remark & Substrate & Depth (m) & Location & country & sea & Reference \\
\hline EL & 0.267 & 3.75 & & & & & \begin{tabular}{|l|} 
Artificial reef: blocks made \\
from stabilized coal-fired \\
power station waste materials \\
(stabilized Pulverized Fuel Ash \\
(PFA))
\end{tabular} & -10 & Poole Bay & $\begin{array}{l}\text { United } \\
\text { Kingdom }\end{array}$ & The English Chanal & Jensen et al. (1994) \\
\hline EL & 0.006 & 166.67 & 79 & 65 & 114 & Summer & $\begin{array}{l}\text { extensive areas of rock and } \\
\text { cobble and soft substarte as } \\
\text { well }\end{array}$ & $\begin{array}{r}-27.2(-16.7- \\
31.8) \\
\end{array}$ & $\begin{array}{l}\text { Northumberland coast, } \\
\text { Blyth }\end{array}$ & UK & North Sea & Skerritt et al. (2012) \\
\hline EL & 0.002 & 500.00 & 79 & 65 & 114 & Winter & soft/mixed habitat & $\begin{array}{r}-27.2(-16.7- \\
31.8) \\
\end{array}$ & \begin{tabular}{|l|}
$\begin{array}{l}\text { Northumberland coast, } \\
\text { Blyth }\end{array}$ \\
\end{tabular} & UK & North Sea & Skerritt et al. (2012) \\
\hline EL & 0.007 & 142.86 & 82 & 64 & 114 & Summer & $\begin{array}{l}\begin{array}{l}\text { extensive areas of rock and } \\
\text { cobble and soft substrate as } \\
\text { well }\end{array} \\
\end{array}$ & $\begin{array}{r}-27.2(1-6.7- \\
31.8)\end{array}$ & $\begin{array}{l}\text { Northumberland coast, } \\
\text { Blyth }\end{array}$ & UK & North Sea & Skerritt (2014) \\
\hline EL & 0.00037 & 2702.70 & $\sim 80$ & $<47$ & $\sim 150$ & \begin{tabular}{|l|} 
Using total water \\
surface of \\
Oosterschelde $(350$ \\
$\left.\mathrm{km}^{2}\right)$
\end{tabular} & $\begin{array}{l}\text { dyke protection of bolders, } \\
\text { cobble. }\end{array}$ & 0 to $-48,80$ & Oosterschelde & Netherlands & Oosterschelde & $\begin{array}{l}\text { Van Stralen and } \\
\text { Smeur (2008) }\end{array}$ \\
\hline EL & 0.060 & 16.62 & $\sim 80$ & $<47$ & $\sim 150$ & \begin{tabular}{|l} 
Using total dyke \\
length $(240 \mathrm{~km})$ and \\
an average depth of 9 \\
$\mathrm{~m}$
\end{tabular} & $\begin{array}{l}\text { dyke protection of bolders, } \\
\text { cobble. }\end{array}$ & 0 to $-48,80$ & Oosterschelde & Netherlands & Oosterschelde & $\begin{array}{l}\text { Van Stralen and } \\
\text { Smeur (2008) }\end{array}$ \\
\hline AL & 0.155 & 6.45 & & 11 & 83 & & $\begin{array}{l}\text { Artificial reef: rocks, } 5-100 \text { an } \\
\text { in diameter up to } 15 \text { an thiok } \\
\end{array}$ & -18 & Northumberland Strait & Canada & Gulf of St. Lawrence & Scarratt (1973) \\
\hline AL & 0.178 & 5.62 & & & & $\begin{array}{l}\text { Natural lobster } \\
\text { grounds }\end{array}$ & \begin{tabular}{|l|} 
glacial till: coarse sand and \\
gravel
\end{tabular} & & Northumberland Strait & Canada & Gulf of St. Lawrence & Scarratt (1973) \\
\hline AL & 0.34 & 2.94 & & $<75$ & $>150$ & & $\begin{array}{l}\text { Artificial reef: pumice concrete } \\
1 \text { and 3-chambers }\end{array}$ & -6.4 & Point Judith, Rhode Island & Canada & \begin{tabular}{|l|} 
Northumberland \\
Strait, Gulf of St. \\
Lawrence
\end{tabular} & Sheehy (1976) \\
\hline AL & 0.227 & 4.41 & & & & & \begin{tabular}{|l|} 
Breakwater wall of the Harbor \\
of Refuge with strong tidal \\
currents, no significant wave \\
action, coarse sand $300 \mu$ \\
\end{tabular} & -5.6 & Point Judith, Rhode Island & Canada & $\begin{array}{l}\text { Northumberland } \\
\text { Strait, Gulf of St. } \\
\text { Lawrence }\end{array}$ & Sheehy (1976) \\
\hline AL & 0.065 & 15.38 & $\geq 50$ & 50 & & $\begin{array}{l}\text { mid-summer peak } \\
\text { density }\end{array}$ & $\begin{array}{l}\begin{array}{l}\text { cobble/boulder, ledge, soft } \\
\text { sediment }\end{array} \\
\end{array}$ & -30 & $\begin{array}{l}\text { Vinalhaven Island, at the } \\
\text { mouth of PenobscotBay }\end{array}$ & Maine, USA & Atlantic Ocean & $\begin{array}{l}\text { Dunnington et al. } \\
(2005)\end{array}$ \\
\hline AL & 0.09 & 11.11 & 66 & 50 & $<83$ & end of august & Cobble & -30 & $\begin{array}{l}\text { Carvers Harbor, Vinalhaven } \\
\text { Island } \\
\end{array}$ & Maine, USA & Atlantic Ocean & \begin{tabular}{|l|} 
Dunnington et al. \\
$(2005)$
\end{tabular} \\
\hline $\mathrm{AL}$ & 0.065 & 15.38 & 66 & 50 & $<83$ & end of august & Ledge & -30 & $\begin{array}{l}\text { Carvers Harbor, Vinalhaven } \\
\text { Island } \\
\end{array}$ & Maine, USA & Atlantic Ocean & \begin{tabular}{|l|} 
Dunnington et al. \\
$(2005)$
\end{tabular} \\
\hline $\mathrm{AL}$ & 0.04 & 25.00 & 66 & 50 & $<83$ & end of august & Sediment & -30 & \begin{tabular}{|l|} 
Carvers Harbor, Vinalhaven \\
Island \\
\end{tabular} & Maine, USA & Atlantic Ocean & \begin{tabular}{|l|} 
Dunnington et al. \\
$(2005)$
\end{tabular} \\
\hline $\mathrm{AL}$ & 0.026 & 38.46 & $\geq 83$ & 83 & & end of august & Cobble & -30 & \begin{tabular}{|l|} 
Carvers Harbor, Vinalhaven \\
Island \\
\end{tabular} & Maine, USA & Atlantic Ocean & \begin{tabular}{|l|} 
Dunnington et al. \\
$(2005)$
\end{tabular} \\
\hline $\mathrm{AL}$ & 0.002 & 500.00 & $\geq 83$ & 83 & & end of august & Ledge & -30 & \begin{tabular}{|l|} 
Carvers Harbor, Vinalhaven \\
Island \\
\end{tabular} & Maine, USA & Atlantic Ocean & \begin{tabular}{|l|} 
Dunnington et al. \\
$(2005)$
\end{tabular} \\
\hline $\mathrm{AL}$ & 0.005 & 200.00 & $\geq 83$ & 83 & & end of august & Sediment & -30 & \begin{tabular}{|l|} 
Carvers Harbor, Vinalhaven \\
Island \\
\end{tabular} & Maine, USA & Atlantic Ocean & \begin{tabular}{|l|} 
Dunnington et al. \\
$(2005)$
\end{tabular} \\
\hline $\mathrm{AL}$ & 0.18 & 5.56 & 66 & 50 & $<83$ & Mid-july & Cobble & -30 & $\begin{array}{l}\text { Sheep Ledges, Vinalhaven } \\
\text { Island }\end{array}$ & Maine, USA & Atlantic Ocean & \begin{tabular}{|l|} 
Dunnington et al. \\
$(2005)$
\end{tabular} \\
\hline
\end{tabular}




\begin{tabular}{|c|c|c|c|c|c|c|c|c|c|c|c|c|}
\hline AL & 0.053 & 18.87 & 66 & 50 & $<83$ & Mid-july & Ledge & -30 & $\begin{array}{l}\text { Sheep Ledges, Vinalhaven } \\
\text { Island }\end{array}$ & Maine, USA & Atlantic Ocean & \begin{tabular}{|l} 
Dunnington et al. \\
$(2005)$
\end{tabular} \\
\hline AL & 0.004 & 250.00 & 66 & 50 & $<83$ & Mid-july & Sediment & -30 & $\begin{array}{l}\text { Sheep Ledges, Vinalhaven } \\
\text { Island }\end{array}$ & Maine, USA & Atlantic Ocean & $\begin{array}{l}\text { Dunnington et al. } \\
(2005)\end{array}$ \\
\hline AL & 0.028 & 35.71 & $\geq 83$ & 83 & & \begin{tabular}{|l|} 
Mid-july \\
\end{tabular} & Cobble & -30 & $\begin{array}{l}\text { Sheep Ledges, Vinalhaven } \\
\text { Island }\end{array}$ & Maine, USA & Atlantic Ocean & \begin{tabular}{|l}
$\begin{array}{l}\text { Dunnington et al. } \\
(2005)\end{array}$ \\
\end{tabular} \\
\hline AL & 0.023 & 43.48 & $\geq 83$ & 83 & & Mid-july & Ledge & -30 & $\begin{array}{l}\text { Sheep Ledges, Vinalhaven } \\
\text { Island }\end{array}$ & Maine, USA & Atlantic Ocean & $\begin{array}{l}\begin{array}{l}\text { Dunnington et al. } \\
\text { (2005) }\end{array} \\
\end{array}$ \\
\hline AL & 0.005 & 200.00 & $\geq 83$ & 83 & & Mid-july & Sediment & -30 & $\begin{array}{l}\text { Sheep Ledges, Vinalhaven } \\
\text { Island }\end{array}$ & Maine, USA & Atlantic Ocean & $\begin{array}{l}\text { Dunnington et al. } \\
(2005)\end{array}$ \\
\hline AL & 0 & & 66 & 50 & $<83$ & Mid-august & Cobble & -30 & $\begin{array}{l}\text { Browns Head, Vinalhaven } \\
\text { Island }\end{array}$ & Maine, USA & Atlantic Ocean & \begin{tabular}{|l} 
Dunnington et al. \\
$(2005)$
\end{tabular} \\
\hline AL & 0.156 & 6.41 & 66 & 50 & $<83$ & Mid-august & Ledge & -30 & $\begin{array}{l}\text { Browns Head, Vinalhaven } \\
\text { Island }\end{array}$ & Maine, USA & Atlantic Ocean & \begin{tabular}{|l} 
Dunnington et al. \\
$(2005)$
\end{tabular} \\
\hline AL & 0.016 & 62.50 & 66 & 50 & $<83$ & Mid-august & Sediment & -30 & $\begin{array}{l}\text { Browns Head, Vinalhaven } \\
\text { Island }\end{array}$ & Maine, USA & Atlantic Ocean & $\begin{array}{l}\text { Dunnington et al. } \\
\text { (2005) }\end{array}$ \\
\hline AL & 0 & & $\geq 83$ & 83 & & Mid-august & Cobble & -30 & $\begin{array}{l}\text { Browns Head, Vinalhaven } \\
\text { Island }\end{array}$ & Maine, USA & Atlantic Ocean & \begin{tabular}{|l} 
Dunnington et al. \\
(2005)
\end{tabular} \\
\hline AL & 0.017 & 58.82 & $\geq 83$ & 83 & & Mid-august & Ledge & -30 & $\begin{array}{l}\text { Browns Head, Vinalhaven } \\
\text { Island }\end{array}$ & Maine, USA & Atlantic Ocean & \begin{tabular}{|l} 
Dunnington et al. \\
$(2005)$
\end{tabular} \\
\hline AL & 0.006 & 166.67 & $\geq 83$ & 83 & & Mid-august & Sediment & -30 & $\begin{array}{l}\text { Browns Head, Vinalhaven } \\
\text { Island }\end{array}$ & Maine, USA & Atlantic Ocean & \begin{tabular}{|l} 
Dunnington et al. \\
(2005)
\end{tabular} \\
\hline AL & 0.09 & 11.11 & 66 & 50 & $<83$ & end of august & Cobble & -30 & $\begin{array}{l}\text { Dogfish Island, Vinalhaven } \\
\text { Island }\end{array}$ & Maine, USA & Atlantic Ocean & $\begin{array}{l}\text { Dunnington et al. } \\
(2005)\end{array}$ \\
\hline AL & 0.066 & 15.15 & 66 & 50 & $<83$ & end of august & Ledge & -30 & $\begin{array}{l}\text { Dogfish Island, Vinalhaven } \\
\text { Island }\end{array}$ & Maine, USA & Atlantic Ocean & \begin{tabular}{|l} 
Dunnington et al. \\
$(2005)$
\end{tabular} \\
\hline AL & 0.037 & 27.03 & 66 & 50 & $<83$ & end of august & Sediment & -30 & $\begin{array}{l}\text { Dogfish Island, Vinalhaven } \\
\text { Island }\end{array}$ & Maine, USA & Atlantic Ocean & $\begin{array}{l}\text { Dunnington et al. } \\
\text { (2005) }\end{array}$ \\
\hline AL & 0.016 & 62.50 & $\geq 83$ & 83 & & end of august & Cobble & -30 & $\begin{array}{l}\text { Dogfish Island, Vinalhaven } \\
\text { Island }\end{array}$ & Maine, USA & Atlantic Ocean & \begin{tabular}{|l} 
Dunnington et al. \\
$(2005)$
\end{tabular} \\
\hline AL & 0 & & $\geq 83$ & 83 & & end of august & Ledge & -30 & $\begin{array}{l}\text { Dogfish Island, Vinalhaven } \\
\text { Island }\end{array}$ & Maine, USA & Atlantic Ocean & $\begin{array}{l}\text { Dunnington et al. } \\
(2005)\end{array}$ \\
\hline AL & 0.007 & 142.86 & $\geq 83$ & 83 & & end of august & Sediment & -30 & $\begin{array}{l}\text { Dogfish Island, Vinalhaven } \\
\text { Island }\end{array}$ & Maine, USA & Atlantic Ocean & $\begin{array}{l}\text { Dunnington et al. } \\
\text { (2005) }\end{array}$ \\
\hline
\end{tabular}




\subsection{Reproduction and juveniles}

\subsubsection{Mating}

In most areas lobsters do not mature before 5-8 years and males reach sexual maturity earlier than females (depending on water temperature). Lobster are long-lived and iteroparous, capable of reproducing many times. Size at functional and physiological maturity of females also varies geographically presumably related to temperature. Females can start bearing eggs at $71 \mathrm{~mm}$. The size range of mature females (bearing eggs) found in the wild ranged from 71 (mostly North Sea) to 150 $\mathrm{mm} \mathrm{CL}$. The range of estimates of $\mathrm{L}_{50}$ for functionally mature female lobster from different locations range from 95 to $140 \mathrm{~mm}$ (Figure 11, Contarini et al., 2008, Lizarraga - Cubedo et al., 2003, Laurans et al., 2009, Phillips, 2013, Wood, 2018).

The lobster mating system is polygynous whereby larger, competitively dominant males are more successful in obtaining mating shelters and in courting females. Also larger claws lead to higher mating success (Phillips, 2013, Sørdalen et al., 2018). Male $H$. americanus have sperm present in the vas deferens at $45-50 \mathrm{~mm} \mathrm{CL}$, but given their small size mating is not likely to occur yet. Data for $H$. gammarus are not available. Reproduction takes place during summer (around July) and is linked with the moulting cycle. Mating usually takes place shortly after the female molts; a spermatophore is deposited internally by the male in the seminal receptacle of the female. Sperm may be stored for several years. After extrusion, the eggs are held on the pleopods for approximately another year until hatching the following summer. In general, moulting cycles are two to three years from smaller to larger females. Large females (>120 mm carapace length) have been shown to moult and then undergo two successive spawns before moulting again, suggesting the capacity for sperm storage. Both Homarus species egg sizes are large, clutch sizes small, and brood period long relative to other marine decapods of similar size (e.g., spiny lobsters and crabs) (Prodöhl et al., 2006, Phillips, 2013). Clutch sizes range from a few thousand to 20,000 larvae, increasing with $\mathrm{CL}$ and age (Contarini et al., 2008, Figure 11).

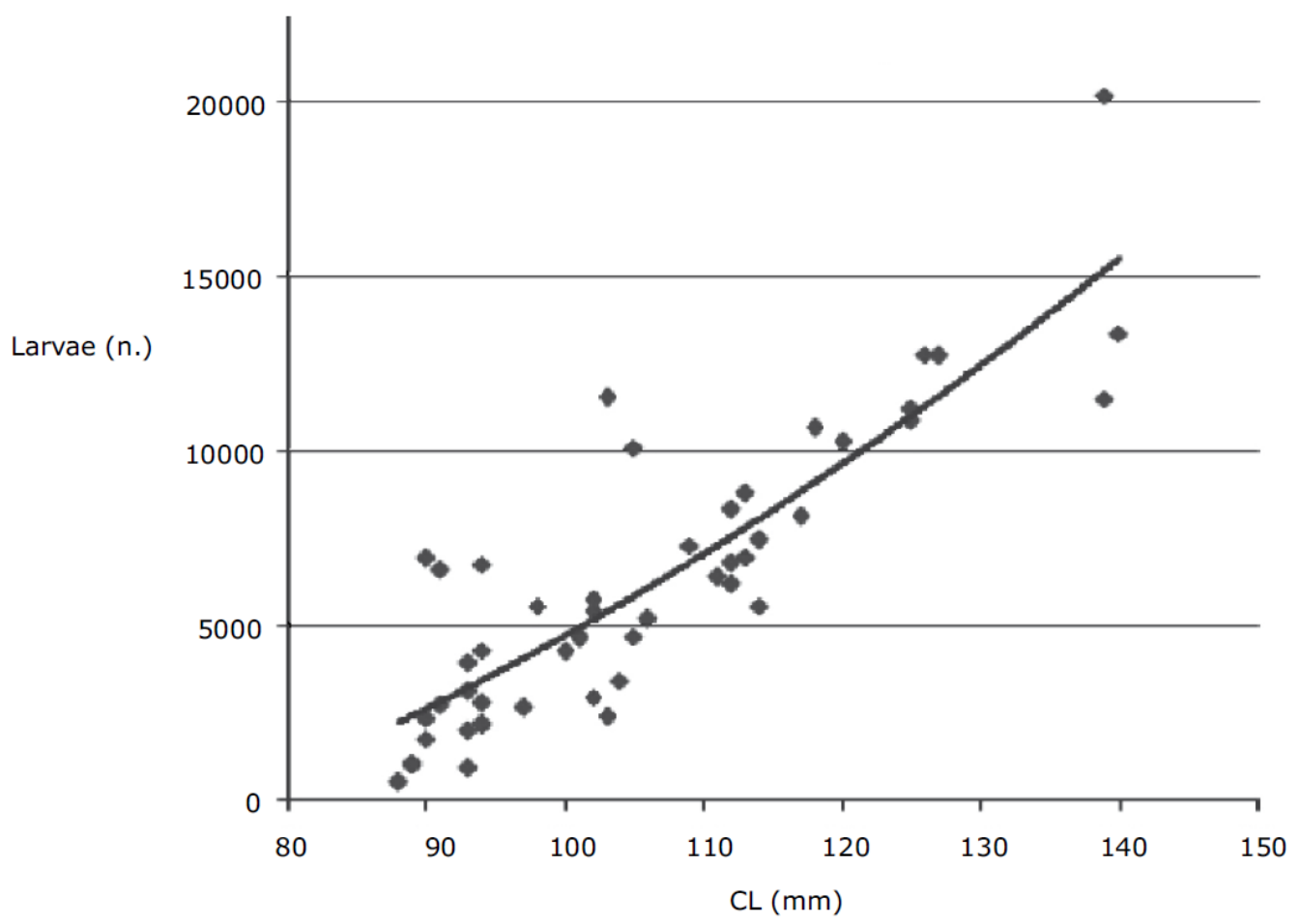

Figure 11. Number of hatched larvae (stage I) in function of maternal carapace length (CL) (taken from Contarini et al., 2008). 


\subsubsection{Pelagic larval stage}

The first few post-hatching weeks are characterised by a pelagic phase usually lasting 10-18 days depending on water temperature (three larval instars and one post larval instar are pelagic)(Wickins \& Lee, 2002, Skerrit, 2014, Figure 12). During this period, larvae undergo metamorphosis to a lobsterlike body at the molt from Stage III to post larva. There is a distinct change in body-structure, with the claws and tail moving from their dangling positions to point out straight from the thorax and abdomen respectively. This alteration towards the post-larval form of an adult allows the post larva to swim forwards using the pleopods and seek a suitable substrate to settle (gravel or coarse sand).

Literature and transport models suggest that most larvae are generally transported between 6 to $<100$ km (Jensen et al., 1994, Krone \& Schröder, 2011, Phillips, 2013). Local currents and residual currents seem to determine distribution. Local availability of substrate and benthic habitat determine settlement. For example, patterns of post larval concentration predicted for the Gulf of St. Lawrence bore little resemblance to spatial patterns in the harvest, suggesting other factors, such as the availability of benthic habitat, may ultimately limit adult abundance (Phillips, 2013).

Larval production and transport are critical components of the dynamics of lobster populations. It has been estimated that only $0.005 \%$ of the hatchling lobsters survive the planktonic phase to reach the benthic phase (Phillips, 2013)2.

\subsubsection{Early settlement}

The metamorphosis is accompanied by changes in behaviour and habitat preference. Two to four days after metamorphosis to post larva bottom-seeking behaviour appears, in order to find appropriate nursery habitat (gravel or coarse sand). Then the post larva makes the transition from pelagic to benthic environment and becomes a permanent resident of the sea bed. Based on indirect measurements and extrapolations, larval settlement strength of $H$. gammarus is associated with local sea temperatures (production and mortality) and onshore winds (transport and availability) as is described for $H$. americanus as well (Sheehy and Bannister, 2002, Phillips, 2013). Mercer et al. (2001) observed that predation on unprotected EBO lobsters was generally rapid. They suggested that time can be a critical factor during settlement, and high mortality can occurs where suitable shelter or substrate is unavailable.

The juvenile phase of the life cycle is characterized by an early shelter-restricted period in which the lobster emerges infrequently from its burrow, followed by an increase in range of movement and habitat choice. In the beginning young $H$. gammarus are cryptic and spend their initial year of life almost totally underground in burrows feeding on infauna and possibly bacteria (Table 4). Small lobsters are well adapted to a burrowing existence. However at a certain moment the food reserves within and close to the burrow become increasingly incapable of providing complete sustenance. Food quantity, availability and nutritional variety within the burrow are major factors influencing when the juvenile lobster emerges to forage. Lobsters then starts to forage further afield seeking shelter whenever necessary to avoid strong currents, competition and predators. At this stage they will eat marine worms and other post-larval animals like small crabs, urchins and gastropods, as well as retaining the ability to filter-feed on plankton. In general at a carapace length of about $15 \mathrm{~mm}$ they leave their burrows for crevices in rocky substrate to begin life as an adult. (Howard, 1988, Jensen et al., 2000, Phillips, 2013, ${ }^{2}$ ).

Despite multiple and widespread investigations, no extensive information is currently available on the early benthic phase (EBP) of the European lobster from settlement at $5-7 \mathrm{~mm} \mathrm{CL}$ until $20 \mathrm{~mm}^{+}$and juveniles are hardly found up to $40-45 \mathrm{~mm} \mathrm{CL}$. It is not clear whether they simply are uncommon, or rarely settle in densities high enough to be detected. Alternatively, they could be in unexplored habitats. While densities of American lobster (H. americanus) juveniles are commonly found and used in stock assessments, it is not feasible to use EBP or early juveniles to predict future recruitment in $H$. gammarus. (Mercer et al., 2001, Prodöhl et al., 2006, Phillips, 2013). 


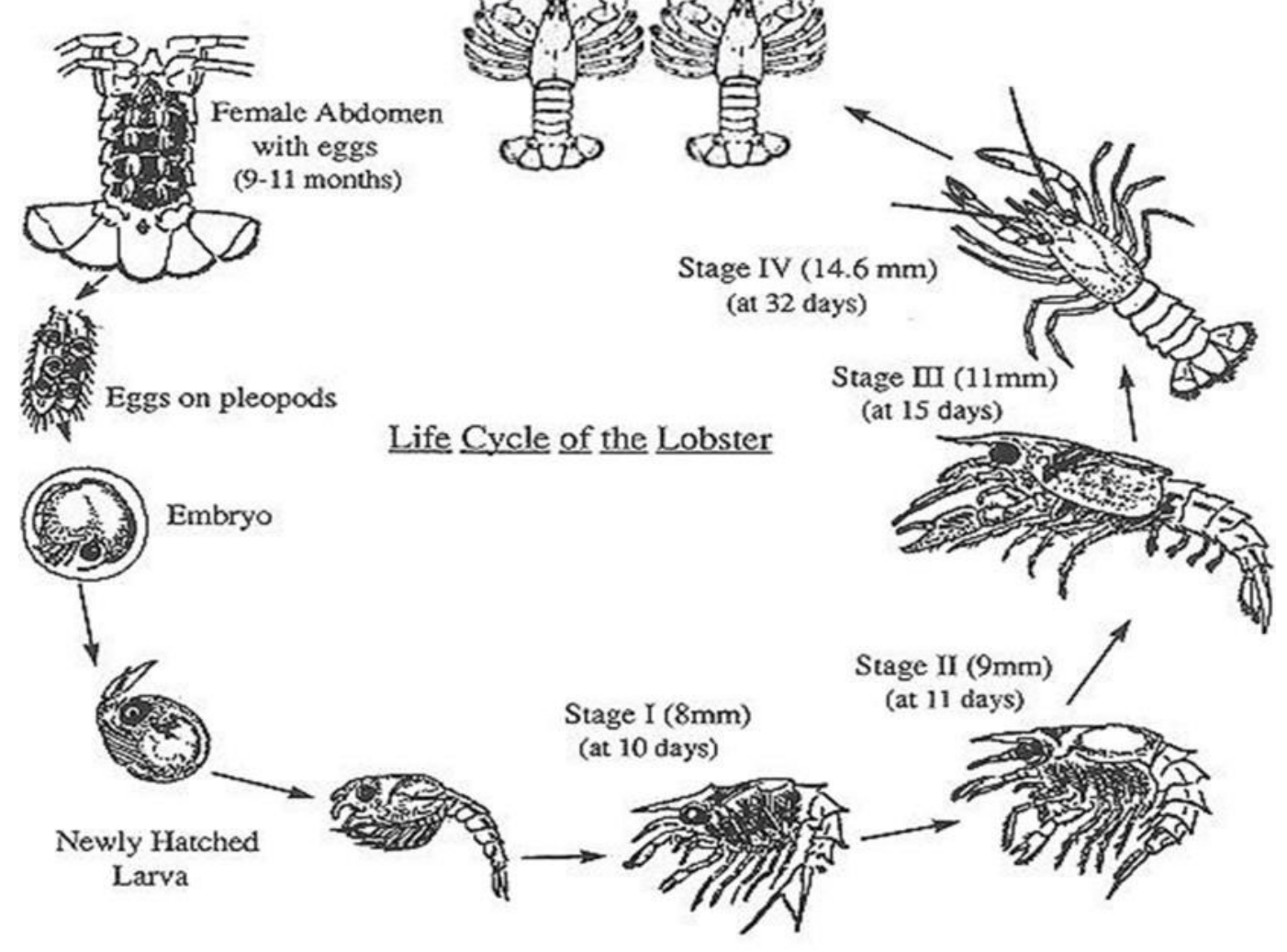

Figure 12. The life cycle of Homarus spp. Newly hatched larva are the beginning of the pelagic stage, and stage III represents the ontogenetic shift to a benthic stage (from Skerrit, 2014).

\subsubsection{Refugia}

Sheehy et al (1999) suggested that the lobster's natural longevity can be interpreted as a life history optimization strategy to enhance reproductive success. Larger (older) females do have higher number of eggs (Figure 11). This emphasizes the demographic importance of old lobsters as reproductive strongholds in closed areas like e.g. wrecks or selected OWFs more offshore. The meta-population analysis of $H$. americanus suggested that heavily fished inshore stocks could depend critically on larval input from offshore. Given the slow growth and reproduction these population 'refugia' could take many years to replace when fished out. This may also explain why some stocks have never recovered from heavy exploitation despite significant effort reduction. Refugia can also serve as safeguards for the genetic diversity (e.g. males and females can grow to larger sizes thereby safeguarding genes for larger growth, Sørdalen et al., 2018)

\subsection{Diet}

There is little detailed data on the diet of $H$. gammarus, while more information can be found for $H$. americanus. Benthic American lobsters are omnivorous, feeding on a great range of benthic organisms. The diet spectrum encompasses a broad range of plants and animals (even algae and zooplankton) but is dominated by molluscan, crustacean, fish, echinoderm, and polychaete remains (Table 12 in Annex 2). They are also cannibalistic. The range of prey species and amount consumed changes as a function of lobster size (age), temperature, prey availability, habitats, season and molt cycle (Holthuis, 1991, Elner \& Campbell 1987, Sainte-Marie and Chabot, 2002, Hanson, 2009). At one location mussels (Modiolus modiolus, Mytilus edulis) appeared the most consistently important identified prey species, in terms of frequency-of-occurrence and estimated volume (points) indexes, while at another location crustaceans were the most important species (Hanson, 2009). 
Lobsters eat until satiated hiding any left-over food for consumption at a later time. The growth and survival of juvenile lobsters is greater where there is a consistent and plentiful food supply, the latter occurring as it precludes the need for risky excursions in pursuit of food (Phillips, 2013).

There is some debate on whether $\mathrm{H}$. americanus is a selective feeder or not. Elner and Campbell (1987) state that lobsters are selective feeders with a complex foraging behaviour and they are capable of maintaining gross diet composition despite strong variations in prey availability. Others think that lobsters are either simply opportunistic omnivores whose 'stomach contents reflect the relative abundance of prey species in the habitat' (Miller et al. 1971,) or 'scavengers' (Herrick, 1895, cited by Elner and Campbell, 1987) and 'most unspecialized feeders' consuming 'almost anything, regardless of its possible nutritional value.

For this study it is sufficient to conclude that bivalves are part of the lobster diet. Blue mussel is mentioned (Elner \& Campbell 1987, Sainte-Marie and Chabot, 2002, Hanson, 2009).

\subsection{Mobility}

Mobility of European lobster can be divided in daily activities and more migratory movements. European lobsters are sedentary animals with migration ranges varying from 0 to $45 \mathrm{~km}$. They prefer to remain at the locations where they have settled and are living. In general $H$. gammarus has restricted movements around its territory; $<4 \mathrm{~km}$ for periods of up to a year (Figure 13, Jensen et al. 1994, Jensen et al., 2000, Smith et al., 2001, Skerrit et al., 2015). On a daily basis $H$. gammarus seems to make short random movements away from shelter with a maximum range of $125 \mathrm{~m}$ (Skerrit et al., 2015, Lees et al., 2018), which could be influenced by local competition for food, shelter and mates. They are capable of fast propulsion using their telson, but cannot maintain this momentum, relying on walking for sustained movements. E.g. H. americanus typically walks in five minute bouts; with a mean walking speed of $0.9 \mathrm{~m} \mathrm{~min}^{-1}$, increasing to $2.5 \mathrm{~m} \mathrm{~min}^{-1}$ (Skerrit, 2014). In term of long term movement and migration, Bannister et al. (1994) observed most recaptures of tagged lobsters within $6 \mathrm{~km}$ of known release positions. Only a small number of larger individuals have been observed to travel up to $15.7 \mathrm{~km}$ in a season (Jensen et al., 1994) or even up to $45 \mathrm{~km}$ (Smith et al., 2001), presumably finding new territories.

As an illustration the findings reported in Skerrit et al. (2015) are discussed in more detail. They observed seasonal patterns in activity: an utilisation distribution ranged from 244 to $7,722 \mathrm{~m}^{2}$ during spring (mean \pm SE: $11,104 \pm 397 \mathrm{~m}^{2}$ ), and declined to $237-784 \mathrm{~m}^{2}$ during autumn (mean \pm SE: 455 $\pm 66 \mathrm{~m}^{2}$ ). They also found behavioural differences between sexes, with males using more space than females. Both sexes conduct the majority of their activities within similar areas, male lobsters had a greater propensity for travelling further from shelter and were more likely to have multiple cores of usage (Figure 13). Taking the diversity in habitat use and high individual variation is seems that populations are governed by individual 'personalities traits' in the form of variation in boldness, habitat-use, exploration and movement which in turn are likely governed by environmental opportunities and individual fitness. As demonstrated in Figure $13 \mathrm{H}$. gammarus travels over soft habitat. Studies of colonisation by lobsters of an artificial reef in the UK, positioned $3 \mathrm{~km}$ away from suitable lobster ground, also show that ability (Jensen et al., 1994).

On population level, both adults and larvae are considered to be able to use wrecks, OWFs and other hard substrates like oil and gas platforms as stepping stones to reach all parts of the North Sea (Krone \& Schröder, 2011). 


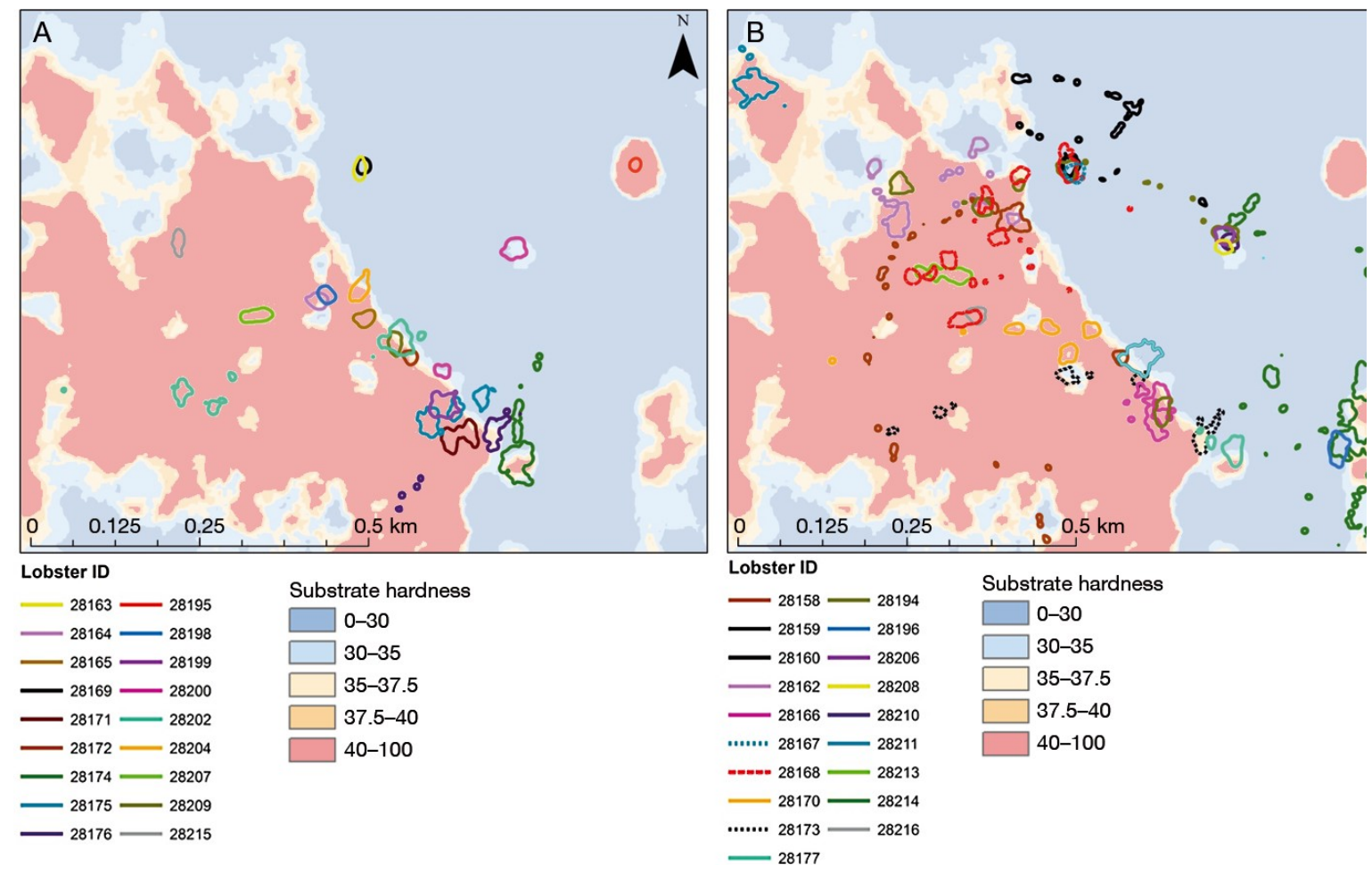

Figure 13. 95\% utilisation distribution (95UD) home-ranges of (A) 18 female and (B) 19 male European lobsters during the spring study period (from Skerrit et al., 2015)

\subsection{Population genetics}

H. gammarus can be divided into six to seven genetically distinct clusters. Ellis et al. (2017)

distinguished four genetic cluster (Figure 14): An Atlantic and Swedish cluster (with high connectivity) and in between those a transitional zone within the eastern North Sea, and an isolated Aegean cluster. The transitional zone between the Atlantic and Swedish cluster suggests an isolation by distance and suggests that direct gene exchange between these stocks is limited which fits a stepping-stone model. Prodöhl et al. (2006) and Agnalt et al. (2009) suggested two more distinct populations: one in the north of Norway (the "midnight-sun lobster") and another one in the Oosterschelde. Triantafyllidis et al. (2005) suggested even two different subpopulations in the north of Norway. Though the separation between the two clusters is only $142 \mathrm{~km}$ of shoreline, probably the hydrological characteristics of the two fjord systems prevent sufficient exchange of larvae. Taking into consideration the potential for migratory behaviour and larval dispersion, genetic exchange between European lobster populations seems unexpectedly restricted (see also Krone \& Schröder, 2011).

Based on the low degree of genetic differentiation revealed in the European lobster and its limited capacity for dispersal, Triantafyllidis et al. (2005) suggested that all populations have been established from a common refuge after the end of the last Ice Age ( $<15,000$ years ago). The northern Norway, Netherlands and Aegean groups exhibit reduced gene diversity (Triantafyllidis et al., 2005, Prodöhl et al., 2006). Although the overall level of genetic differentiation among European lobster populations is low, still the genetic differences seem important adaptive aspects. It is extremely likely that lobsters living at the edges of environmental tolerance for the species are adapted to some degree to these differences in conditions (Triantafyllidis et al., 2005, Prodöhl et al., 2006, Agnalt et al., 2009). 

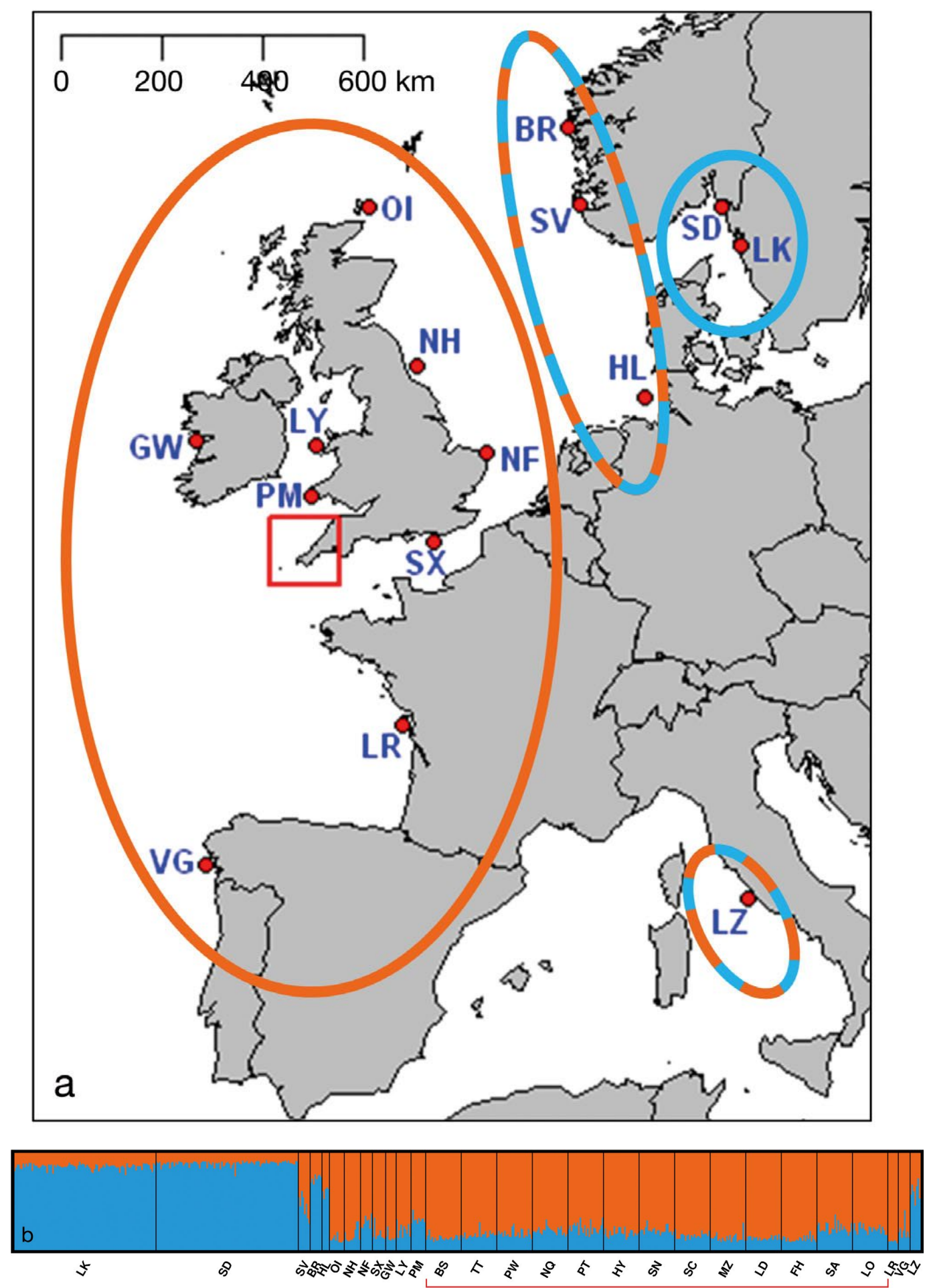

Figure 14. Assignment and map of population clusters. (a) Sample groupings as indicated by colour composition of individual samples (vertical bands) in (b). (b) Destruct plot of 5 converged iterations of $\mathrm{K}=2$ using a priori location data. The inset red area in (a) and red underlined section in (b) denote fine-scale samples from Cornwall, UK. (Ellis et al., 2017).

\subsection{Enhancement of stocks}

Lobster production can be increased actively (aquaculture) or passively (enhancing local stocks). For aquaculture two forms can be distinguished: product enhancement (wild caught lobsters are maintained in pounds where they are fed to improve quality/size). And secondly there is full growout (close cycle culture): rearing lobsters from egg to marked size. Until recently, full grow-out 
culture of lobsters was not considered economically viable given the logistical implications related to the need to keep individual lobsters in separate compartments due to their cannibalistic behaviour (Wickins \& Lee, 2002) and the lack of automated procedures for feeding and maintenance. In optimal rearing conditions (e.g. at constant $20^{\circ} \mathrm{C}$ ), it is possible to rear a portion size lobster $(250-300 \mathrm{~g}$, total length $210 \mathrm{~mm}, \mathrm{CL} 75 \mathrm{~mm}$, Prodhöl et al., 2006 or $345-400 \mathrm{~g}$, Wickins \& Lee, 2002) from hatching in 800-900 days.

For enhancing local stocks three strategies have been defined: habitat enhancement, stock enhancement or food enhancement, which will be described in more detail below.

\subsubsection{Habitat enhancement}

Habitat enhancement has several aspects: 1) increasing the volume and surface available for lobsters 2 ) increasing the volume and surface available for food, and 3) offering crevices and caves which are suitable for all sizes lobsters emphasising especially the larger lobsters (Howard, 1988, Jensen et al., 1994, 2000, Wickins \& Lee, 2002). When lobsters are present in the neighbourhood they will colonize the suitable new substrate rapidly by immigration of adult specimen not depending on larval settlement (Scarratt, 1973, Sheehy, 1976, Jensen et al., 1994, 2000, Phillips, 2013). The antiscouring of OWF monopiles is considered to be good potential new artificial habitat which can support lobsters (Linley et al, 2008, Buck et al., 2017, Roach et al., 2018). However not always European lobsters are encountered at such locations, raising doubts on suitability of the location or reachability (Bouma \& Lengkeek, 2012, Hooper \& Austen, 2014). Since the Dutch NCP is rather scarcely occupied with hard substrates, adding extra substrates could improve the potential for populations of $H$. gammarus, using e.g. the designs as mentioned in Jensen et al. (2000) or Buck et al. (2017). Lobsters themselves can learn us on the requirements of the habitats, e.g. on a cohesive clay/mud substrate, juvenile $H$. americanus create hydro dynamically advantageous burrow systems that facilitate the exchange of oxygenated water (Lawton and Lavalli, 1995). Optimum habitats for $H$. gammarus will therefore include mature, heterogeneous cobble-boulder layers overlaying penetrable substrate offering crevices for all sizes of lobsters (Howard, 1988, Jensen, 2000, Wickins \& Lee, 2002).

The designs and materials used provide lobster habitat worldwide range from specially designed, new concrete (Reef Ball type), artificial reefs designed and built by engineers from non-waste materials, or unspecific casually deposited large, constructed from low-cost 'materials of opportunity' (like rock rubble or stabilised, pulverised fuel ash) (Wickins \& Lee, 2002, Rozemeijer et al., 2017). More research is needed to get more insights of the required spatial needs (hydrodynamic conditions, tolerable nearest neighbour distances, foraging behaviour) and habitat needs (crevice size and shape preferences, food availability) and thus carrying capacity of a reef structure for lobsters of different sizes to survive and grow within a defined area (Sheehy, 1976, Wickins \& Lee, 2002). To illustrate demands on habitats and their positioning, habitat enhancement constructs in Canada having their entrances towards the main hydrological forces were tumbled over after storms whereas otherwise oriented constructs remained unaffected (Sheehy, 1976, Wickins \& Lee, 2002).

Monitoring is needed within OWFs to determine the local populations of European lobsters to determine whether this form of enhancement can be of service to enhance local productivity.

\subsubsection{Stock enhancement}

As is mentioned in sections 2.6.2 and 2.6.3 the pelagic stages and early benthic stages seem especially vulnerable. Therefore frequent hatchery programmes have been started in order to increase local populations both for $H$. gammarus as well as $H$. americanus, in order to improve local fisheries and restore stocks (Bannister and Addison, 1998, Jensen et al., 2000, Wickins \& Lee, 2002, Phillips, 2013). These lobster hatcheries can be aiming at hatching eggs, and releasing stage I or stage IV larvae to supplement wild stocks (Prodhöl et al., 2006) or release more full grown animals at a size of e.g. $50 \mathrm{~mm}$ TL (Buck et al., 2017). Currently, programmes are in development to train the juvenile lobsters to enhance survival when being transferred from hatchery with low competition for food and shelter to the natural environment with food shortage, predators etc. (e.g. Agnalt et al., 2017). Despite the mobility of larvae and adults, (re)colonisation can be reduced, therefore stock 
enhancement could be an option (Hooper \& Austen, 2014, Buck et al., 2017). It seems important to use local lobsters in order not to have genetic pollution or less adapted animals to local circumstances (Ellis et al., 2017).

An important issue to tackle, before starting stock enhancement, is the cost-benefit analyses which should encompass aspects like:

- $\quad$ objectives of the release programme;

- $\quad$ scale of the operation;

- ownership rights;

- $\quad$ policy on population genetics and ecological impact of the released species;

- $\quad$ size and number of hatcheries;

- $\quad$ size at which the crustaceans are to be released;

- $\quad$ social benefits and externalities.

It can be worthwhile to do stock enhancement. A female lobster can have up to 20,000 eggs (Figure 11 ), however only one of these is expected to survive in the wild. Stock enhancement techniques are thought to improve this survival rate by about 1000 times. However a large investment in numbers released seems needed to improve stocks at the scale of areas. Bannister and Addison (1998) suggested a release of 10000 lobsters per year on area level for 5 years, to demonstrate a significant measurable result. They were quit critical on claiming potential success. Others do see potential improvement of local populations (Wickins \& Lee, 2002, Duffill-Telsnig, 2014, Buck et al., 2017). The National Lobster Hatchery Cornwall claims a $>30 \%$ increase in lobster catchments whereas catchments on other crustaceans are declining due to their efforts ${ }^{2}$.

Monitoring on focussing on juvenile lobsters is needed within OWFs to determine the local populations of European lobsters to determine whether this form of enhancement can be of service to enhance local productivity.

\subsubsection{Food enhancement}

In principle $H$. gammarus has a broad diet, yet food availability might be limiting. Given the large size and energy density of the flesh, an ample supply is necessary. In the TKI Win-Wind project food enhancement is one of the enhancement strategies to be investigated. Food enhancement can be achieved more passively: 1 increase of hard substrate and habitat; or by actively introducing 2 . favoured food; or 3. extra food in the local environment. Fishery discards that are now to be landed without direct purpose could be a cheap source.

Seeding with e.g. the favoured mussels could promote food stock present. Disadvantage is e.g. that mussels can attract other predators too like fish and starfish (Wickins \& Lee, 2002,). In addition seeding actively could destabilize a local system that is developing a certain direction due the prevailing (a)biotic circumstances at that moment.

Adding extra mussels to enhance local populations of favoured species or e.g. fisheries discards nearby could reduce the risks for lobsters running out of food. In addition feeding time could be enhanced when having food nearby due to providing discards nearby. Lobsters in particular cannot forage in high current speeds (Howard, 1988). Close supply of e.g. discards could reduce the need to forage away from the burrow, save foraging time and walking, and thereby extend the period of time over which they can safely feed before tide currents are too strong $\left(>0.6 \mathrm{~m} \mathrm{~s}^{-1}\right.$, speeds that can occur on the Dutch NCP, Howard, 1988, Wickins \& Lee, 2002). 


\section{$3 \quad$ Other relevant aspects for modelling}

This short chapter gives the relevant data needed for the modelling of lobster on monopiles. The characteristics of the OWF Princess Amalia are treated in addition to a an example of habitat enhancement: Reef Balls.

\subsection{OWF Princess Amalia}

The aim is to model the growth of lobster in OWFs at monopile antiscouring. To this end information from the existing OWF Princes Amalia is used, although information of other parks is also available (Figure 1). OWF Princess Amalia was selected for a number of reasons, namely:

1. In this OWF a concrete test on lobster and brown crab fishing will start (TKI project WinWind).

2. Already data are available on the biodiversity and biomasses on the anti-scouring and other hard substrate are available.

3. In 2019 once again the anti-scouring and other hard substrate will be measured.

4. It is integral part of the planned OWF Hollandse Kust Noord-Holland (HK-NH) (Figure 1).

5. Being located at 19-24 $\mathrm{m}$ depth, it is in the transition zone between near shore and offshore.

6. I is also reasonably close to the measuring station (bottom lander, Witbaard et al., 2014) from which data are used to derive as natural as possible annual developments of ChIA, SPM and $\mathrm{T}$.

Relevant aspect for OWF Prinses Amalia are given in Table 6 and Table 7. The most relevant aspect is that the total anti-scouring surface in the park is $0.149 \%(0.073 \%$ for Luchterduinen) a minimal amount so the local consumption will hardly influence the primary production.

Table 6. Relevant aspects of OWF Prinses Amalia and OWF Luchterduinen (data obtained from Eneco). The data of OWF Luchterduinen are added because for the TKI project WinWind OWF Prinses Amalia is choosen and OWF Luchterduinen is the alternative.

\begin{tabular}{|c|c|c|c|}
\hline Aspect & Unit & Prinses Amalia OWF & Luchterduinen \\
\hline Number of windmills & $\mathrm{N}$ & $\begin{array}{l}60 \text { (All } 61 \text { have scour } \\
\text { protection included } \\
\text { substation) }\end{array}$ & $\begin{array}{c}43 \text { (ONLY } 42 \text { have SCOUR protection } \\
\text { included OHVS) }\end{array}$ \\
\hline $\begin{array}{l}\text { Average distance } \\
\text { between the monopoles }\end{array}$ & $\mathrm{m}$ & $550-600$ & 650 \\
\hline Capacity of the OWF & MW & 120 & 129 \\
\hline Depth & $\mathrm{m}$ & $19-24$ & $19-26$ \\
\hline Distance to the shore & $\mathrm{km}$ & 23 & 23 \\
\hline Diameter scouring & $\mathrm{m}$ & 21 & 18 \\
\hline $\begin{array}{l}\text { Surface area } \\
\text { antiscouring per pole }\end{array}$ & $\mathrm{m}^{2}$ & 346.4 & 260.2 \\
\hline $\begin{array}{l}\text { Characterisation of anti- } \\
\text { scouring }\end{array}$ & & $\begin{array}{l}\text { one layer of filter with an } \\
\text { armour layer of rocks on } \\
\text { top of it. The median rock } \\
\text { diameter is around } 0.5 \mathrm{~m} \text {. }\end{array}$ & $\begin{array}{l}0,3 \mathrm{~m} \text { layer of filter layer with an armour } \\
\text { layer of } 1.2 \text { meter. The rock layer has } \\
\text { boulders of a median diameter of } 40 \mathrm{~cm}\end{array}$ \\
\hline $\begin{array}{l}\text { Average size of crevices } \\
\text { (width*height*depth) }\end{array}$ & $\mathrm{cm}$ & $\begin{array}{l}8 \times 8 \times 8 \text { assume perfect } \\
\text { circle }\end{array}$ & $6 \times 6 \times 6$ assuming perfect circle \\
\hline Range width of crevices & $\mathrm{cm}$ & $8-9$ & $6-8$ \\
\hline Range height of crevices & $\mathrm{cm}$ & $8-9$ & $6-8$ \\
\hline Range depth of crevices & $\mathrm{cm}$ & $8-9$ & $6-8$ \\
\hline
\end{tabular}




\begin{tabular}{|l|l|c|c|}
\hline Aspect & Unit & Prinses Amalia OWF & Luchterduinen \\
\hline $\begin{array}{l}\text { Total Surface area Anti- } \\
\text { scouring OWF }\end{array}$ & $\mathrm{m}^{2}$ & 21128 & 10927 \\
\hline Surface OWF (km2) & $\mathrm{km}^{2}$ & 14,218 & 15,035 \\
\hline Surface OWF (m2) & $\mathrm{m}^{2}$ & 14218000 & 15035000 \\
\hline $\begin{array}{l}\text { \% anti-scouring vs total } \\
\text { are OWF }\end{array}$ & $\%$ & $0.149 \%$ & $0.073 \%$ \\
\hline
\end{tabular}

Table 7. Abiotic aspects of some selected existing and planned OWFs: sea bed shear stress, suspended particles, water temperature (winter minimum and summer maximum), sea bed structure and motion, sediment composition (from Smaal et al., 2017).

\begin{tabular}{|c|c|c|c|c|c|c|c|c|c|}
\hline \multirow[t]{2}{*}{ OWF } & \multicolumn{3}{|c|}{$\begin{array}{l}\text { sea bed shear stress } \\
(\mathrm{N} / \mathrm{m} 2)\end{array}$} & \multicolumn{2}{|c|}{$\begin{array}{l}\text { suspended } \\
\text { matter }(\mathrm{mg} / \mathrm{l})\end{array}$} & \multicolumn{2}{|c|}{$\begin{array}{l}\text { water } \\
\text { temperature }\left({ }^{\circ} \mathrm{C}\right)\end{array}$} & \multirow{2}{*}{\begin{tabular}{|c|} 
sea bed \\
motion
\end{tabular}} & \multirow[t]{2}{*}{$\begin{array}{l}\text { sediment } \\
\text { composition }\end{array}$} \\
\hline & $\max$ & $\begin{array}{c}\text { max wave } \\
\text { action }\end{array}$ & avg & $\max$ & avg & $\min$ & $\max$ & & \\
\hline Borssele & 2 & 2 & 0,6 & 25 & 10 & 4 & 20 & 0 (local) & coarse to fine sand \\
\hline $\mathrm{HK}-\mathrm{ZH}$ & 4 & 5 & 0,6 & 35 & 10 & 4 & 18 & $?$ & fine sand \\
\hline OWEZ & 8 & 8 & 0,8 & 50 & 20 & 3 & 20 & $?$ & fine sand \\
\hline $\mathrm{HK}-\mathrm{NH}$ & 5 & 6 & 0,6 & 35 & 10 & 4 & 18 & $?$ & fine sand \\
\hline Luchterduinen & 5 & 6 & 0,6 & 35 & 10 & 3 & 20 & $?$ & fine sand \\
\hline Prinses Amalia & 5 & 7 & 0,6 & 35 & 10 & 3 & 18 & $?$ & fine sand \\
\hline Buitengaats & 4 & 7 & 0,4 & 40 & 10 & 3 & 18 & $?$ & fine sand \\
\hline Zee-energie & 4 & 6 & 0,3 & 40 & 10 & 3 & 18 & $?$ & silty sand \\
\hline
\end{tabular}

\subsection{Habitat enhancement through Reef Balls}

In order to enhance the habitat several options are possible that can serve as a lobster enhancing habitat (Jensen et al, 2000). The most important aspects are that several size (age) classes of lobster can be accommodated. Especially the younger stages need the protection of the crevice. Also the shape of the crevice is preferably elongated (Jensen et a., 2000, Linley et al., 2007). However, enhancing habitat for a large lobster is not easily defined given the harsh conditions near the bottom of the NCP. E.g. the monopiles need location specific anti-scouring protection with strict design rules (Lengkeek et al., 2017). Also placing constructions on the anti-scouring or directly on the sandy bottom needs a careful approach (Raaijmakers, Deltares, pers. comm.).

In the Netherlands extensive research is performed using e.g. Reef Balls as an example of general habitat improvement. Given the fact that we are currently in the preliminary stages of constructing an ecological habitat for lobster, Reef Balls might serve as an example and the characteristics can be included in our calculations. Natural reefs are variable in size, shape and hole density. Artificial reefs function better when they mimic nature. Reef Balls can be created with varying hole sizes to host differently sized lobsters. Reef Balls are made by pouring concrete into a fiberglass mold containing a central Polyform buoy surrounded by various sized inflatable balls to make holes (Figure 15, Table 8).

In general Reef Balls are designed such that over half of the weight is in the bottom of the ball and near the sea floor. All sizes of Reef Balls have withstood, without movement, heavy tropical storms in as little as 20 feet of water without anchors. Reef Balls are stable because the opening in the top of the unit breaks up the lifting force of the hydrofoil effect common to dome shapes. In essence they are chimneys and an under-pressure is generated by the passing currents thereby promoting stability. Side holes are wider near the centre of the walls and narrow near the units surface. This feature creates miniature vortexes which further reduce lifting forces and bring rich nutrients to organisms living on the reef. 


\begin{tabular}{|c|c|c|c|c|c|c|c|}
\hline Style & Width (m) & $\begin{array}{l}\text { Height } \\
(\mathrm{m})\end{array}$ & Weight (kg) & $\begin{array}{l}\text { Concrete } \\
\text { Volume } \\
\text { (m3) }\end{array}$ & $\begin{array}{l}\text { Surface } \\
\text { Area }\end{array}$ & \# Holes & Mold prices \\
\hline \begin{tabular}{|l} 
Goliath \\
Booster \\
Ring
\end{tabular} & 2 & 1 & $1,818-2,727$ & 1.19 & 16.7 & $15-25$ & \\
\hline Goliath & 1.83 & 1.52 & $1,818-2,727$ & 1.19 & 21.4 & $25-40$ & $\$ 12,000$ \\
\hline Super Ball & 1.83 & 1.37 & 1, $1,818-2,727$ & 1.19 & 17.6 & $22-34$ & $\$ 10,000$ \\
\hline Ultra Ball & 1.68 & 1.31 & $1,591-2,045$ & 0.76 & 13.9 & $22-34$ & $\$ 8,280$ \\
\hline Reef Ball & 1.83 & 1.16 & $1364-1,909$ & 0.57 & 12.1 & $22-34$ & $\sim \$ 8,280$ \\
\hline Pallet Ball & 1.22 & 0.88 & $682-1,000$ & 0.25 & 7.0 & $17-24$ & $\$ 7000$ \\
\hline Bay Ball & 0.91 & 0.61 & $170-341$ & 0.08 & 2.8 & $11-16$ & $\$ 3630$ \\
\hline $\begin{array}{l}\text { Mini-Bay } \\
\text { Ball }\end{array}$ & 0.76 & 0.53 & $68-91$ & $\begin{array}{l}\text { less than } 4 \\
50 \mathrm{lb} \text {. bags }\end{array}$ & & $8-12$ & $\$ 2300$ \\
\hline Lo-Pro & 0.61 & 0.46 & $36-59$ & $\begin{array}{l}\text { less than } 2 \\
50 \mathrm{lb} \text {. bags }\end{array}$ & & $6-10$ & $\$ 1222$ \\
\hline Oyster & 0.46 & 0.30 & $14-20$ & $\begin{array}{r}\text { less than } 1 \\
50 \mathrm{lb} \text {. bag }\end{array}$ & & $6-8$ & $\$ 898$ \\
\hline
\end{tabular}

A

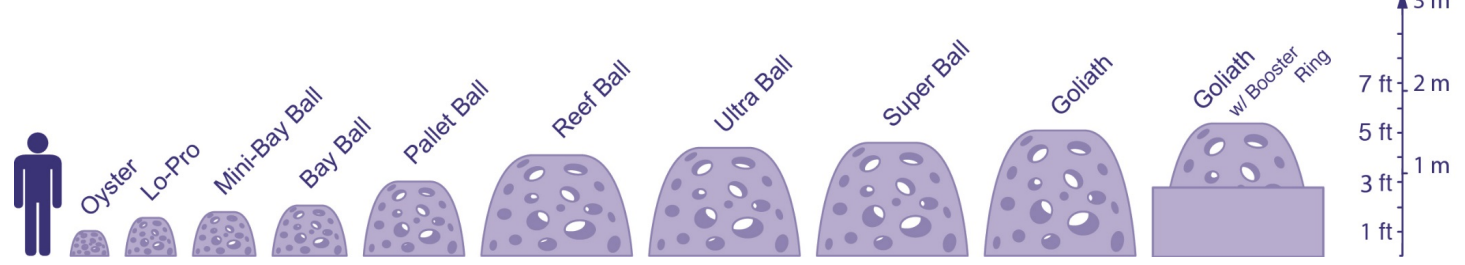

B

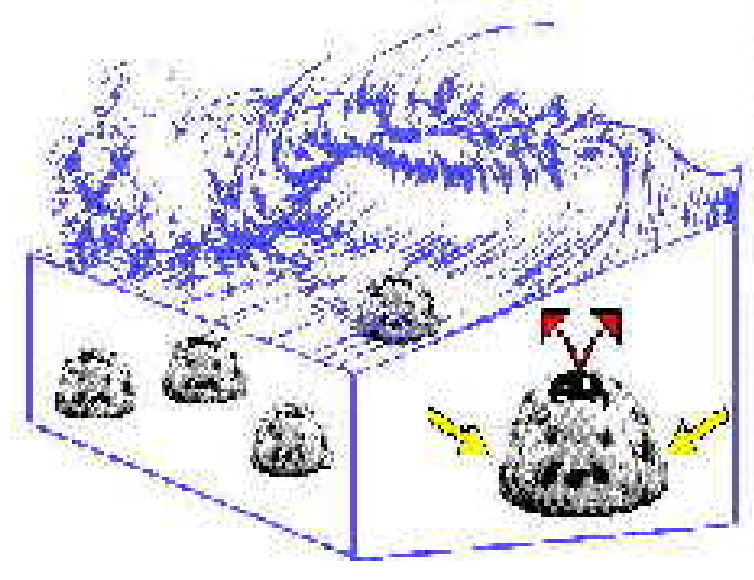

Figure 15. A. Different types of Reef Balls as described in Table 8. B. Chimney functioning of the shape yielding under pressure in the dome of the Reef Ball. 


\section{$4 \quad$ Modelling lobster production}

An energetic population model was developed in order to assess lobster growth on a monopile given seasonal variation in temperature and its effects on lobster rates and resource productivity. Such a model allows for the necessary size based approached since fisheries is a size based activity. In order to incorporate effects of temperature and resource availability, as well as size, a DEB approach was used. Such a model can be used to model individual lobster growth under different circumstances, such as different temperature regimes to mimic different locations. A benefit of this model type is that modelled growth trajectories can be compared to growth trajectories in the field, thereby allowing model validation. In order to predict lobster density at a monopile food competition needs to be considered. To that end, the lobster growth model needs to be incorporated into a population dynamical shell and a dynamically modelled resource needs to be added. The population dynamical shell also allows to study the effects of stocking and differences in resource availability. Habitat enhancement, for example with Reef Balls, could also be incorporated in a later stage.

Alternative modelling approaches are possible, but only a fully size-structured approach, such as DEB, results in growth curves that can be compared to field data. If there is a need to incorporate more food web components a different approach, such as stage-structure, will complexity but will also result in a loss of detail. This is a trade-off that is common to make to ensure feasibility (Lipscius et al., Under Review).

\subsection{Method and parameters}

The production of lobster is modelled for a single monopile. Model results will show lobsters growth and the number of lobsters that can grow given resource availability and temperature regime. Resource and lobster growth are modelled as a function of temperature.

Lobster growth is modelled using a dynamic energy budget (DEB; Kooijman (2009)), which models individual growth and development. Model and parameter values were taken from the Add-My-Pet website (Kooijman and Augustine, 2017) (hereafter referred to as AMP). In order to allow growth to be temperature dependent, the scaling with Arrhenius function was added to the model and accompanying parameter values were estimated. The DEB model does not explicitly include moult.

The individual growth model was embedded in the Escalator Boxcar Train concept (EBT; (de Roos et al., 1992)). With this concept the fate of individuals and their resource can be followed over time, making the population dynamics an emergent property. The EBT model allows for the modelling of a dynamic resource, so that individual energy uptake leads to resource depletion and potentially induces resource competition among individuals. A similar approach was adopted to model the dynamics of brown shrimp (Crangon crangon) along the Dutch coast (Steenbergen et al., 2015). The equations are listed in Table 9 and the parameter values in Table 10.

The resource is modelled following semi-chemostat dynamics, mimicking Mytilus edulis, which is the most abundant species in number, biomass and energy at the anti-scouring surface (Bouma and Lengkeek, 2012). The actual productivity of the resource will vary with temperature. The resource is expressed as caloric content per square meter $\left(\mathrm{J} / \mathrm{m}^{2}\right)$, which feeds into the lobster growth model. The total consumption rate by lobsters, summed over all individuals present, is then subtracted in the resource equation (Table 9 ).

We assume that lobsters are being placed at the monopile at a given body size and this body size is set to the size at settlement or, in case of stocking, to $50 \mathrm{~mm} \mathrm{CL}$. Fishing mortality, or harvesting, is instantaneous and occurs when lobsters reach the minimal landing size of $87 \mathrm{~mm}$ CL Reproduction is accounted for as an energy loss for mature lobsters but there is no natural recruitment occurring at the monopile. This is based on the assumption that the monopile is not a closed system and currents 
will take all offspring elsewhere. Hence, there is no population dynamical feedback through reproduction. New lobsters can only enter the population through stocking. Population dynamics occur only via resource competition.

Table 9. Variables, equations and functions for the shrimp population, physiology and the resources. Index $i$ denotes an individual lobster cohort.

\section{Variables}

Number of cohorts D

Number of individuals in cohort $i$

$$
N_{i}, i \in\{1, D\}
$$

Volume of individuals in cohort $i$

$$
V_{i}
$$

$\mathrm{Jm}^{-2}$

Resource density

\section{Lobster dynamics}

Volume of individuals in cohort $i$

$$
\frac{d V_{i}}{d t}=\kappa P_{E}-M /\left[E_{G}\right] \quad \mathrm{cm}^{3} \mathrm{~d}^{-1} \quad 1
$$

Energy of individuals in cohort $i$

Reproductive energy of individuals in cohort $i$

$\frac{d E_{r, i}}{d t}=(1-\kappa) P_{E}-P_{R}$

Number of individuals in cohort $i$

$$
\frac{d N_{i}}{d t}=-\left(\mu_{s}+\mu_{f}\right) N_{i}
$$

\section{Resource dynamics}

Resource density

$$
\frac{d R}{d t}=r\left(\left(R f_{\tau} K\right)-R\right)-R\left(\sum_{i=1}^{D} S_{m} S A_{\tau} / \text { Area }\right)
$$

\section{Lobster functions}

Maximum intake

Search rate

$$
S=1 /\left(1+R_{j}\left(F_{m} V_{i}^{2 / 3}\right) / I_{m}\right)
$$

Ingestion

$$
I=A_{\tau} S_{m} R_{j} S
$$

$m^{2} d^{-1} \quad 7$

Maintenance

$$
M=A_{\tau}\left[P_{M}\right] V_{i}
$$

Energy utilisation

$$
P_{E}=\frac{E_{i} / V_{i}}{\kappa^{E_{i}} / V_{i}+\left[E_{G}\right]} \cdot\left(\frac{\left(\left\{\mathrm{PXm}{ }^{E_{i}} / V_{i} \varepsilon V_{i}^{\frac{2}{3}}\right)\right.}{\left[E_{M}\right]}\right)+M
$$

Development and maintenance of maturity

$$
P_{R}=\left\{\begin{array}{l}
\frac{V_{i}(1-\kappa)}{\kappa} M \text { if } V_{i} \leq V_{P} \\
\frac{V_{P}(1-\kappa)}{\kappa} M \text { if } V_{i}>V_{P}
\end{array}\right.
$$

Starvation mortality
$C \rightarrow C+1$ 
Instantaneous Fishing mortality

Carapace length

Arrhenius scaling at day $\tau$

$$
\mu_{f}=\left\{\begin{array}{l}
0 \text { if } L_{i}<L_{h} \\
1 \text { if } L_{i} \geq L_{h}
\end{array}\right.
$$

$$
L_{i}=V_{i}^{1 / 3} / \delta_{m}
$$

$\mathrm{cm}$

$$
A_{\tau}=e^{\frac{T A}{T r e f}}-\frac{T A}{T_{\tau}} \cdot \frac{\left(1+e^{\frac{T A L}{T r e f}-\frac{T A L}{T L}}+e^{-\frac{T A H}{T H}-\frac{T A H}{T r e f}}\right)}{\left(1+e^{\frac{T A L}{T_{\tau}}-\frac{T A L}{T L}}+e^{\frac{T A H}{T H}-\frac{T A H}{T_{\tau}}}\right)}
$$

\section{Temperature}

Ambient temperature in area

$$
T_{\tau}=\operatorname{amp} \sin \left(\pi \frac{(\tau-\text { center })}{\text { width }}\right)+o f f s e t
$$

Temperature values were taken from Steenbergen et al. (2015), for the coast. OWF's placed in deeper areas will imply lower temperatures and hence reduced growth rates.

Table 10. Model parameters, there symbol used, value, unit and reference. AMP refers to the Add-My-

\begin{tabular}{|c|c|c|c|}
\hline Maximum search rate & $\mathrm{F}_{\mathrm{m}}$ & $\mathrm{m}^{2} \mathrm{~cm}^{-2} \mathrm{~d}^{-1}$ & AMP \\
\hline Handling & $\mathrm{H}$ & 1 & \\
\hline Maximum ingestion & $\left\{P_{x m}\right\}$ & $250 \mathrm{~cm}^{-2} \mathrm{~d}^{-1}$ & this study \\
\hline $\begin{array}{l}\text { Volume specific } \\
\text { Maintenance }\end{array}$ & {$[\mathrm{PM}]$} & $24.3 \mathrm{~J} \mathrm{~cm}^{-3} \mathrm{~d}^{-1}$ & AMP \\
\hline Cost of growth & {$\left[E_{G}\right]$} & $4400 \mathrm{Jcm}^{-3}$ & AMP \\
\hline $\begin{array}{l}\text { Maximum energy } \\
\text { density }\end{array}$ & {$\left[E_{M}\right]$} & $8279.36 \mathrm{~J} \mathrm{~cm}^{-3}$ & AMP \\
\hline Energy egg & $\mathrm{E}_{\mathrm{B}}$ & 43.9] & Pandian (1970) \\
\hline $\begin{array}{l}\text { Carapace length at } \\
\text { settlement }\end{array}$ & $\mathrm{L}_{\mathrm{j}}$ & $0.5253 \mathrm{~cm}$ & AMP \\
\hline $\begin{array}{l}\text { Carapace length at } \\
\text { harvesting }\end{array}$ & $L_{h}$ & $8.7 \mathrm{~cm}$ & this study \\
\hline Volume at maturation & $V_{p}$ & $105.4 \mathrm{~cm}^{3}$ & AMP \\
\hline Shape coefficient & $\delta_{m}$ & $0.573-$ & AMP \\
\hline Kappa & $\mathrm{K}$ & $0.8959-$ & AMP \\
\hline Conversion efficiency & $\varepsilon$ & $0.8-$ & AMP \\
\hline Starvation mortality rate & $S_{r}$ & $0.5 d^{-1}$ & Steenbergen et al. (2015) \\
\hline Starvation threshold & $\mathrm{St}_{\mathrm{t}}$ & 0.75 & Steenbergen et al. (2015) \\
\hline Arrhenius temperature & $\mathrm{T}_{\mathrm{A}}$ & $8000 \mathrm{~K}$ & AMP \\
\hline Optimum temperature & Topt & $293 K$ & Kristiansen et al. (2004) \\
\hline $\begin{array}{l}\text { Lower boundary of } \\
\text { tolerance range }\end{array}$ & $T_{L}$ & $275 K$ & Kristiansen et al. (2004) \\
\hline $\begin{array}{l}\text { Upper boundary of } \\
\text { tolerance range }\end{array}$ & $\mathrm{T}_{\mathrm{H}}$ & $295 K$ & this study \\
\hline $\begin{array}{l}\text { Rate of decrease at } \\
\text { lower boundary }\end{array}$ & $\mathrm{T}_{\mathrm{AL}}$ & $1 \mathrm{e} 6 \mathrm{~K}$ & this study \\
\hline $\begin{array}{l}\text { Rate of decrease at } \\
\text { upper boundary }\end{array}$ & $\mathrm{T}_{\mathrm{AH}}$ & $1 \mathrm{e} 5 \mathrm{~K}$ & this study \\
\hline
\end{tabular}
Pet entry (Kooijman and Augustine, 2017).

\begin{tabular}{llll}
\hline Parameter & Symbol & Value & Unit \\
\hline
\end{tabular}

\section{Individuals}

\section{Resource}

$\begin{array}{llll}\text { Carrying capacity } \quad \mathrm{K} & 1.8 \mathrm{E} 4 \mathrm{~J} \mathrm{~m}^{-2} \quad \text { this study }\end{array}$




\begin{tabular}{|c|c|c|c|c|}
\hline Parameter & Symbol & Value & Unit & Reference \\
\hline
\end{tabular}

\begin{tabular}{|c|c|c|c|}
\hline Regrowth rate & $r$ & $0.1 d^{-1}$ & Kooijman (2009) \\
\hline Arrhenius temperature & $\mathrm{T}_{\mathrm{A}}$ & $7022 K$ & AMP \\
\hline Optimum temperature & Tref & $293.15 K$ & AMP \\
\hline $\begin{array}{l}\text { Lower boundary of } \\
\text { tolerance range }\end{array}$ & $\mathrm{T}_{\mathrm{L}}$ & $275 K$ & AMP \\
\hline $\begin{array}{l}\text { Upper boundary of } \\
\text { tolerance range }\end{array}$ & $\mathrm{T}_{\mathrm{H}}$ & $296 K$ & AMP \\
\hline $\begin{array}{l}\text { Rate of decrease at } \\
\text { lower boundary }\end{array}$ & $\mathrm{T}_{\mathrm{AL}}$ & $45430 \mathrm{~K}$ & AMP \\
\hline $\begin{array}{l}\text { Rate of decrease at } \\
\text { upper boundary }\end{array}$ & $\mathrm{T}_{\mathrm{AH}}$ & $31376 \mathrm{~K}$ & AMP \\
\hline \multicolumn{4}{|l|}{ A-biotics } \\
\hline Temperature amplitude & amp & $8.07 \mathrm{~K}$ & (Steenbergen et al., 2015) \\
\hline Temperature width & width & $182.4 d$ & (Steenbergen et al., 2015) \\
\hline Temperature center & center & $125.36 d$ & (Steenbergen et al., 2015) \\
\hline Temperature offset & offset & $283.78 \mathrm{~K}$ & (Steenbergen et al., 2015) \\
\hline Monopile surface area & Area & $364 \mathrm{~m}^{2}$ & $\begin{array}{l}\text { this study, (Table 6: OWF } \\
\text { Prinses Amalia) }\end{array}$ \\
\hline
\end{tabular}

The parameterization of the lobster model is taken from AMP (Kooijman and Augustine, 2017), while the values for the Arrhenius function were fitted for this application, based on oxygen consumption rates from Whiteley et al. (1990). The unit of the maximum search rate was converted from $\mathrm{cm}^{-2} \mathrm{~d}^{-1}$ to $\mathrm{m}^{2} \mathrm{~cm}^{-2} \mathrm{~d}^{-1}$ to match the units of the rest of the model and assuming a height of $10 \mathrm{~cm}$ within reach. The value of the maximum ingestion rate was raised to $250 \mathrm{~J} \mathrm{~cm}^{-2} \mathrm{~d}^{-1}$ (instead of $217 \mathrm{~J} \mathrm{~cm}^{-2} \mathrm{~d}^{-}$ $\left.{ }^{1}\right)$ to increase the growth rate such that maturation occurs in better correspondence with field data, with an average of 6 years (Jensen et al., 2000).

For the resource parameter values were based on those of Mytilus edulis, as this is based on biomass the dominant species on the monopiles (Bouma and Lengkeek, 2012). For the regrowth rate $r$ we used 0.1 , the growth rate of Mytilus Edulis (Kooijman, 2009). As we model the resource in terms of energy density, the mean ash free dry weight (AFDW) measured on the monopiles from Bouma and Lengkeek (2012) was converted to $\mathrm{J} / \mathrm{m} 2$, based on the conversion of $22 \mathrm{~J} / \mathrm{mg}$ AFWD (Brey et al., 1988), resulting in a maximum energy density of $1.8 \mathrm{E} 4 \mathrm{~J} / \mathrm{m} 2$.

\subsection{Results}

\subsubsection{DEB stand-alone}

As a test the DEB model for lobster was run under stand-alone conditions, meaning that the growth of a single individual is modelled with ad libitum food and given an annual temperature regime. The carapace length development of a lobster over time follows the expected growth curve, where growth slows down at large size (Figure 16). The annual temperature fluctuations are visible with retarded growth rate in the winter period and increased growth rate in summer. The length at age curve presented is compared to field data (Jensen et al., 2000) based on a mark and recapture experiment at the English coast. The carapace length predicted by the model complies with field observations (Bannister et al., 1994; Sheehy et al., 1999; Jensen et al., 2000). 


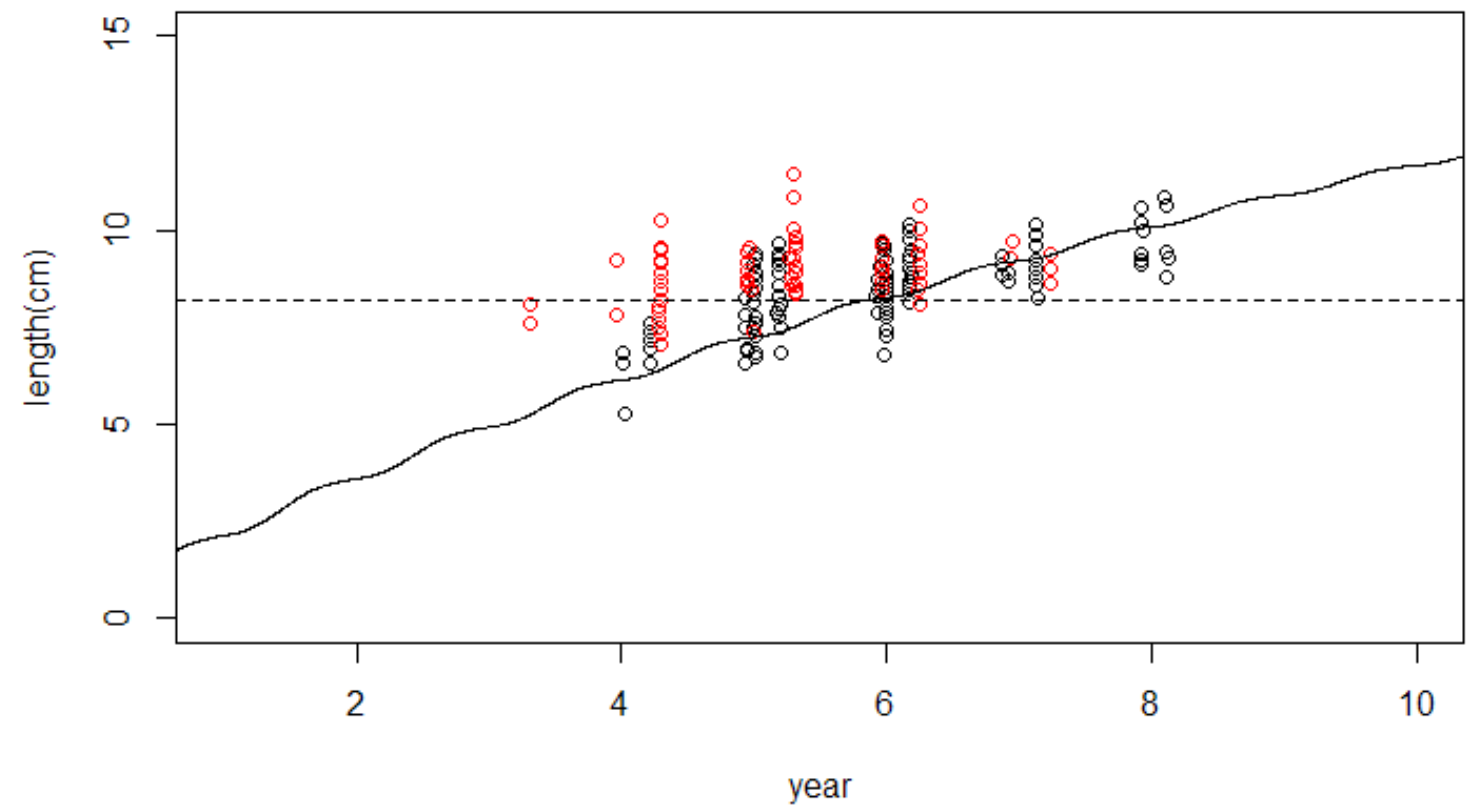

Figure 16. Carapace length $(\mathrm{cm})$ in time for the DEB model (solid line) and length-at-age data taken from (Jensen et al., 2000) (red: 1983-1984; black: 1985-1988). The horizontal dashed lines denotes the size at maturity of $8.2 \mathrm{~cm}$ (AMP value).

From the basic DEB model and its parameters for lobster, one can calculate the time it takes for energy reserves to deplete from maximum energy to zero, for a given CL (Figure 17). This assumes no food intake and starting at the maximum energy density an individual can have given its length. Larger individuals have in total more energy to spend and energy depletion therefore takes longer. Energy depletion is affected by the ambient temperature. In cooler conditions energy depletion is slower than at higher temperatures (Figure 17).

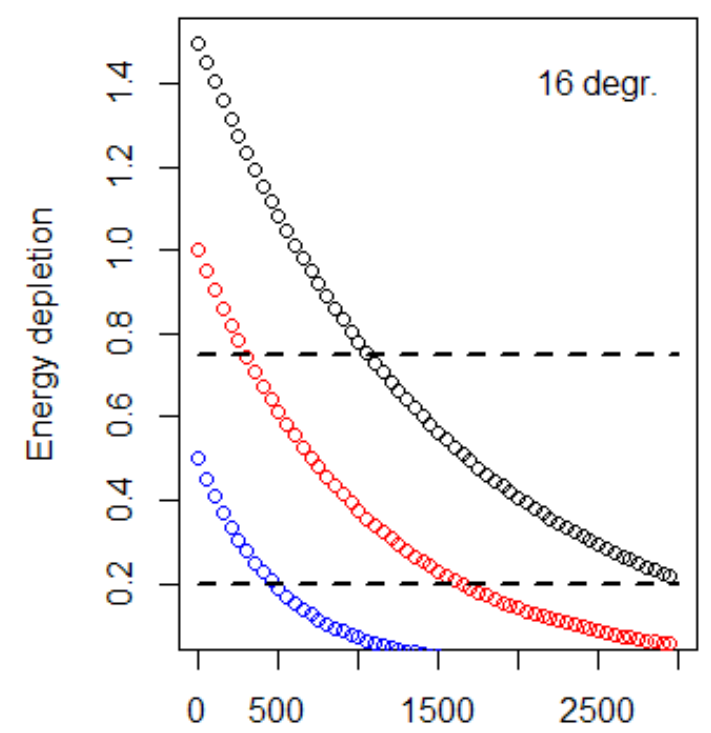

Time (d)

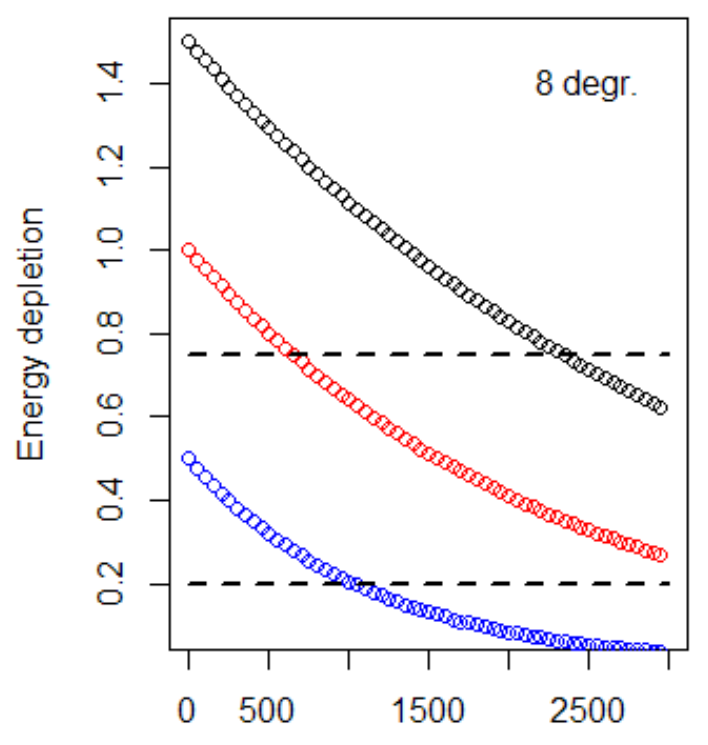

Time (d)

Figure 17. Energy depletion ratio relative to the maximum energy as function of time for different initial carapace lengths (black $=6 \mathrm{~cm}$, red $=4 \mathrm{~cm}$ and blue $=2 \mathrm{~cm}$ ). The left panel shows the depletion at 16 degrees and the right panel at 8 degrees. The horizontal dashed lines denote a depletion of 0.75 and 0.2 . 


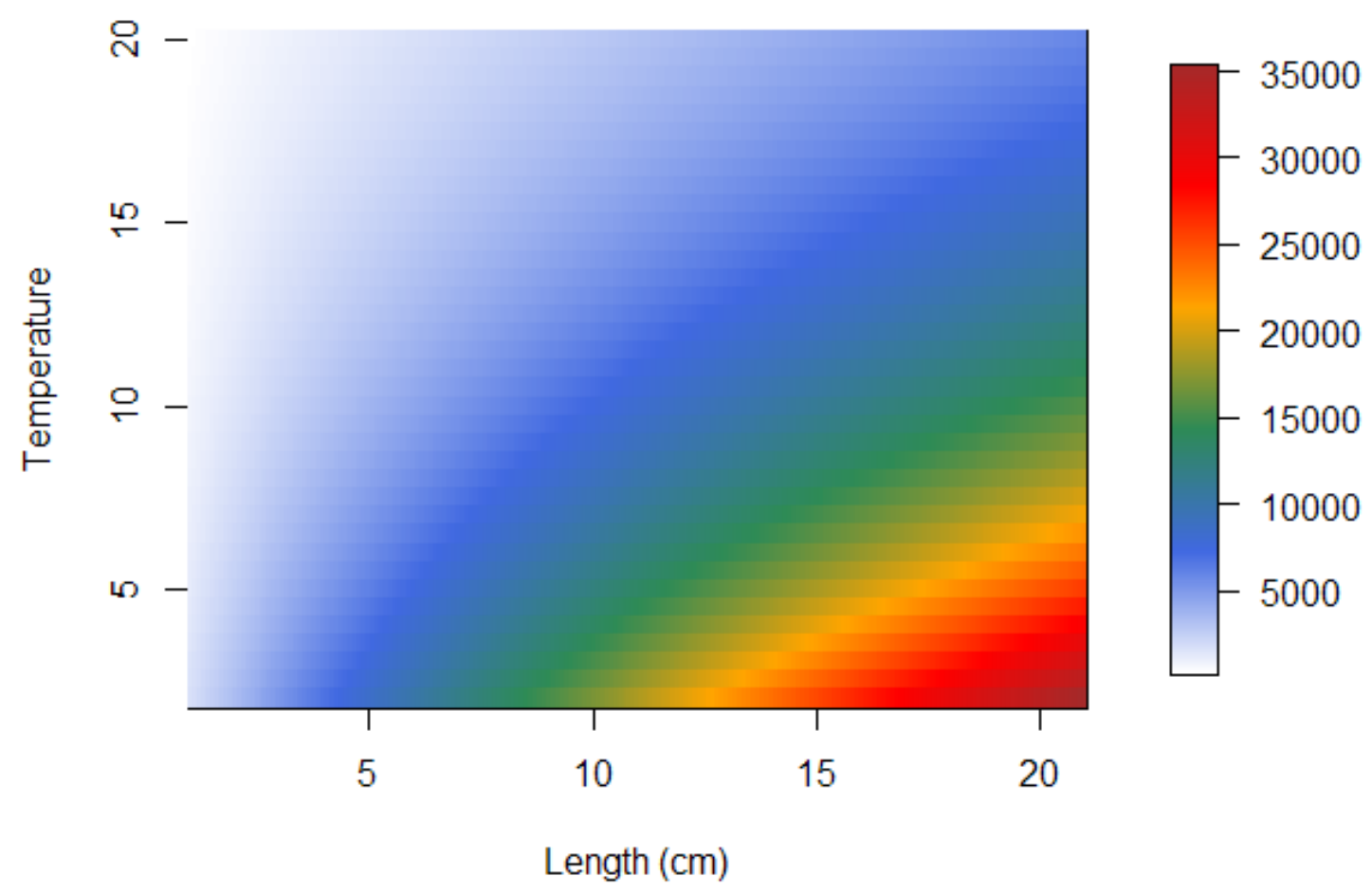

Figure 18. Time (in days, right axis) it takes in days to deplete energy to $20 \%$ of size specific maximum energy as function of carapace length and (constant) temperature (left axis).

From the energy depletion we can defer the time to deplete energy to $20 \%$ of the maximum as a function of length and water temperature (Figure 18). From this information it is clear that larger individuals can survive longer without food than smaller conspecifics. They can sustain lower food conditions due to the high energy content per unit of body weight relative to the maintenance costs per volume times the total amount of body weight available. This difference in survival time is largest at low temperatures. Warmer temperatures decrease the energy depletion time. Overall this result illustrates that lobsters can sustain periods with low resource conditions well.

When food is limited a size difference between individuals results in a competitive difference. Intake rate scales with surface area while maintenance scales with volume (Figure 19). Yet, the increase of both rates with lobster size differs in such manner that intake increases faster than maintenance rate for lobsters smaller than $12 \mathrm{~cm}$. This implies that with constant but low food availability a larger lobster can realize a higher intake than a smaller conspecific, still being able to cover maintenance costs. This provides the larger lobster with a competitive advantage under low food conditions. However, the maintenance rate lobsters larger than $12 \mathrm{~cm} \mathrm{CL}$ increases faster than intake, and therefore the competitive advantage is for the smaller lobsters. This difference in scaling of the rates and the breakpoint (at $12 \mathrm{~cm} \mathrm{CL}$ ) where smaller or larger individuals have a competitive advantage at low food conditions is illustrated by the humped shaped relationship of intake minus maintenance (Figure 19, bottom graph). When comparing the intake rate and the maintenance rate as a function of body size it is clear that intake exceeds maintenance for sizes smaller than $18 \mathrm{~cm} \mathrm{CL}$ (Figure 19). This provides a natural limit where intake and maintenance are balanced. Note that these relationships are based on averages and that natural variability will provide variance around this theoretical value. 

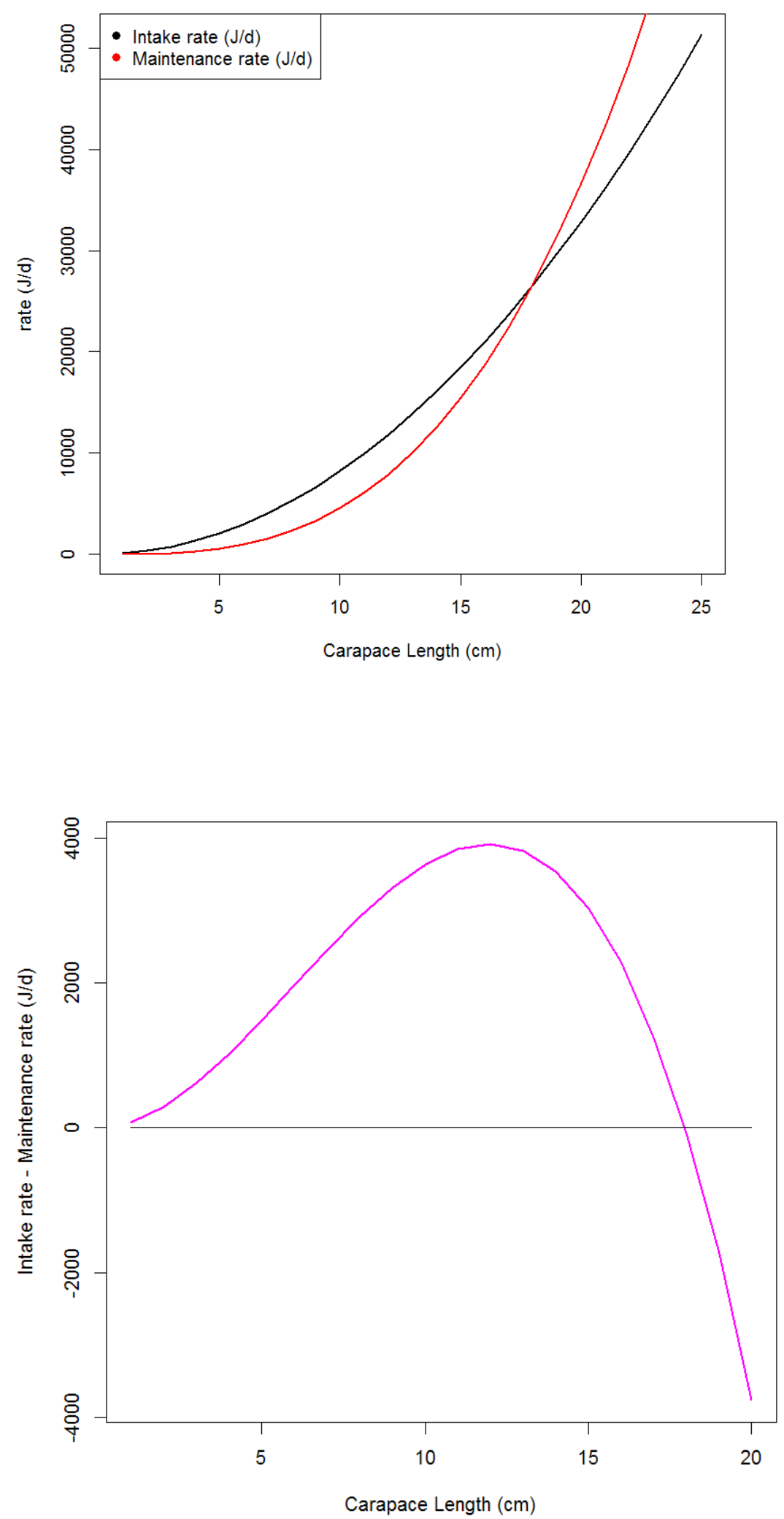

Figure 19. Intake and maintenance rate as function of carapace length (top) and intake minus maintenance cost as a function of carapace length (bottom).

This result from the stand-alone DEB model already suggests that in a population dynamical setting with resource competition larger individuals may outcompete smaller conspecifics and that summer could be the most likely period for starvation mortality to occur. 


\subsubsection{Population dynamical model}

In order to model a dynamic resource and the possibility of resource competition among lobster the DEB model was embedded within a population dynamical framework (de Roos et al., 1992). The growth of a single individual feeding on the benthic resource follows a similar pattern as the DEB stand-alone application (Figure 20). Lobster length increases with time, showing the effect of season with reduced growth in winter. Lobster growth levels off when the resource is depleted such that growth is no longer possible. The size at maturity $(8.2 \mathrm{~cm}, \mathrm{AMP})$ is reached at the end of the fifth year and the size of harvesting $(8.7 \mathrm{~cm}$ for the UK) is reached at the start of the sixth year. The resource follows the temperature driven sinusoidal pattern, yet showing an overall decrease in time due to lobster consumption. After about seven years the resource shows an additional pattern, a second depression in resource density occurring in early summer, which increases and then stabilises as lobster size becomes constant. This depression is caused as temporarily consumption exceeds resource regrowth rate, due to the differences in temperature scaling between lobster and its food (parameter values of the Arrhenius function); i.e. in spring consumption increases faster with increasing temperature than resource growth does. Halfway summer the resource regrowth rate can compensate consumption and resource density increases again. Resource levels are reduced towards an average of $3.5 \mathrm{KJ} / \mathrm{m}^{2}$, in line with benthic productivity estimates in coastal areas (Steenbergen et al., 2015).

When assuming that a lobster is stocked at $5 \mathrm{~cm} \mathrm{CL}$ the results growth curve and resource patterns are identical. With an initial size of $5 \mathrm{~cm}$ the harvesting length is attained after 3 years. 

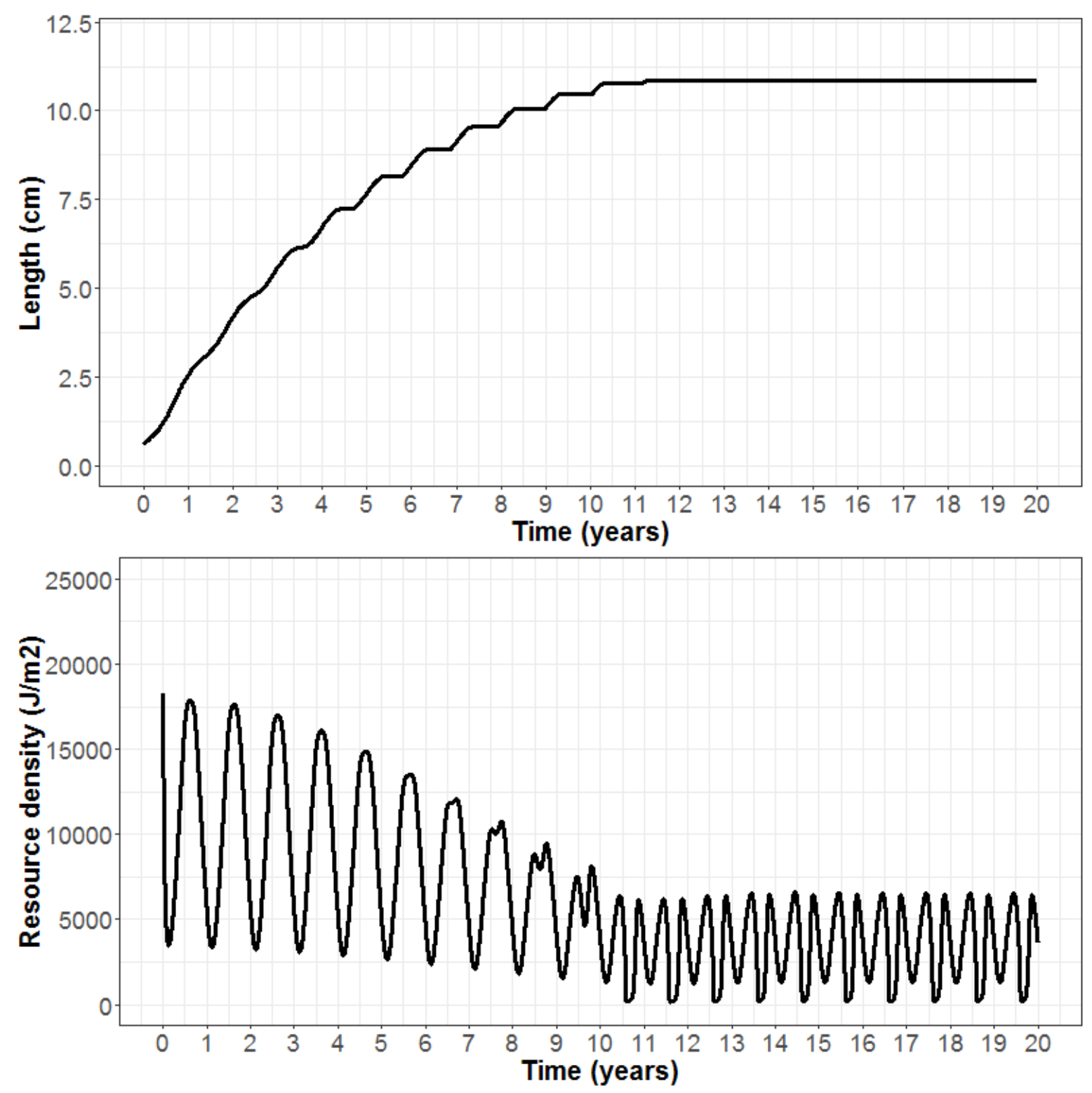

Figure 20. Individual lobster growth (CL top panel) and resource density (bottom panel) resulting from the population dynamics model, initiated with a single lobster.

When more than one lobster is put into the system the attained length decreases due to severe resource depletion. Already with two individuals present the maximum attained size is $8.2 \mathrm{~cm}$, the size of maturation. But this size is below the minimum landing size which prohibits harvesting.

Interestingly, the lobsters do not suffer from starvation mortality, the low resource density suffices to cover the cost of being, but simply does not allow for further growth. If more lobsters are simultaneously introduced, the maximum attained length will be smaller as growth ceases at a smaller size.

Only increased resource productivity will result in increased growth, such that there can be more lobsters that reach harvestable sizes. There is a linear relationship between resource production and lobster growth to harvestable sizes.

\subsection{Restocking}

The model results show that with a single lobster in the system the length of harvesting is reached after 3 years when stocked with a length of $5 \mathrm{~cm} \mathrm{CL}$ (Figure 20). We take therefore 3 years in between stocking events, and stock with individuals of $5 \mathrm{~cm} \mathrm{CL}$. Stocking with lobsters at a smaller 

from the growth curve in Figure 20. Similarly, stocking at a larger length will shorten the time needed at the monopile to reach the minimum harvestable length.
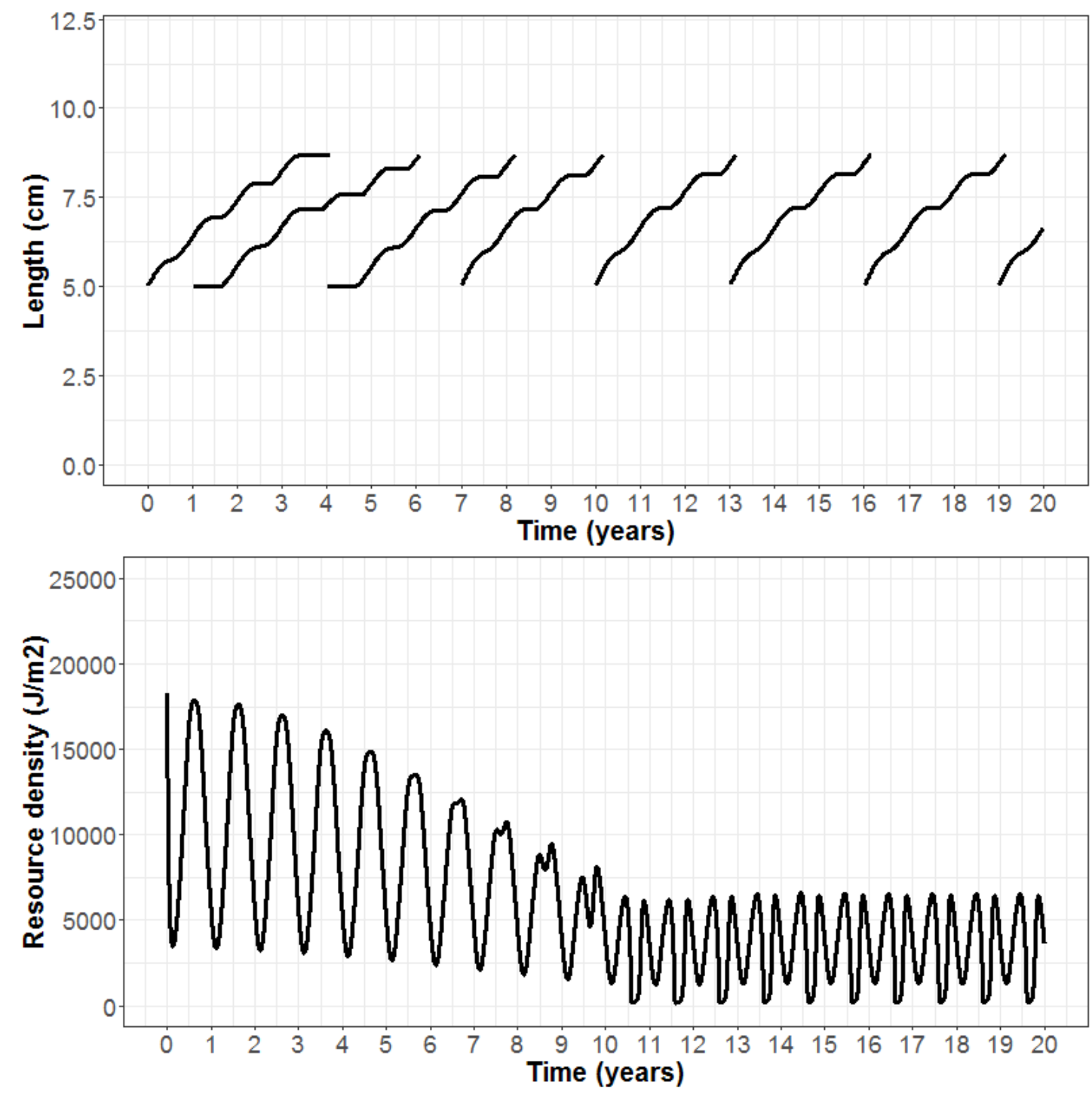

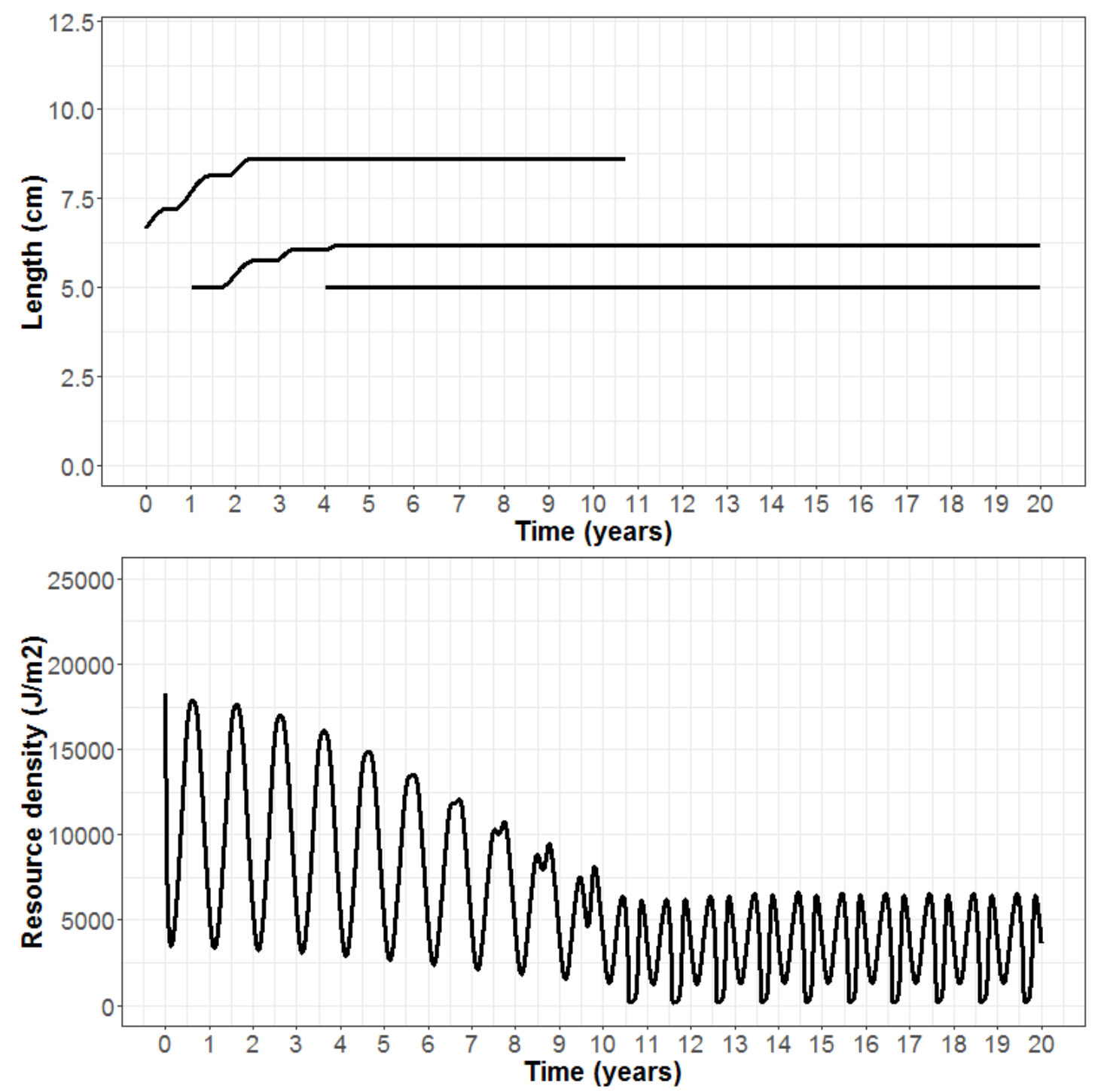

Figure 21. Individual lobster length and resource density in time. From year 1 onwards restocking occurs with lobsters of $5 \mathrm{~cm}$ length. Top graphs: 1 lobster restocking, bottom graphs: 2 lobsters restocking. Lobsters of harvestable lengths $(8.7 \mathrm{~cm})$ are removed from the model.

Figure 21 shows the length of an individual lobster in time, with restocking every three years and removal of harvestable lobsters $(8.7 \mathrm{~cm} \mathrm{CL})$. With one lobster added per stocking event lobsters reach harvestable sizes in three years (Figure 21, top graph). In such a system every three years one lobster can be harvested while one lobster is being placed at the monopile.

When under the same conditions two lobster are introduced at each stocking event, growth stops completely after the first event and the lobsters stocked at the second event do not start to grow (Figure 21, bottom graph). Resource levels are depressed to such levels that the smallest lobsters suffer from starvation mortality, meaning that latest additions starve to death in a few days. This starvation mortality is the result of resource competition between lobsters, which favours larger individuals. This is also demonstrated in Figure 18, where the reserve depletion as function of size and temperature is given. There is a linear relationship between resource productivity and the number of lobsters that reach the minimum landing size. A doubling of the value of carrying capacity doubles the number of lobsters reaching the minimum harvestable size. However, whether or not such increased carrying capacity values still relate to natural systems has to be established.

In case of the current model parameterization there can be one lobster growing from $5 \mathrm{~cm} \mathrm{CL}$ to harvestable length in three years per monopile. This is a density of ca 0.002 individuals $/ \mathrm{m}^{2}$, which is compared to literature on the lower side, but within reported ranges (see Table 5). For American lobster densities in the order of 0.001-3.25 were reported (Howard, 1988; Dunnington et al., 2005). 
Densities vary with temperature, season, productivity and lobster size, e.g. higher densities for smaller sized lobsters (Dunnington et al., 2005).

\subsection{Model discussion}

Any model is by definition a simplification. That said, a model allows for exploration of systems and circumstances that otherwise would be too time consuming, too costly or even impossible to pursue. In this study a DEB model was used both as stand-alone as well as embedded in a population dynamical framework. DEB is a model type with individual size and growth at its core, which allows for ontogenetic difference which in models ignoring size could lead to substantially different results. Above all, ignoring size and growth would mean ignoring one of the most obvious not to mention relatively easy to measure characteristic that defines an individual.

The DEB model shows length development which corroborates with field observations. Interestingly, the lobster energy content and metabolic rate as a function of size are such that larger individuals can sustain periods of resource limitation for a longer period than smaller individuals. Summer appears to be the period for which starvation mortality is most likely to occur if food conditions are poor.

Differences in competitive dominance between size classes occurs due to asymmetry in the increase in maintenance costs vs intake rate as a function of size. When maintenance increases faster with size than intake small individuals are competitively dominant and large individuals win competition when the opposite holds, when intake rate increases faster with size than maintenance. The finding that small lobsters are inferior competitors to larger ones (till $12 \mathrm{~cm}$ ) suggests that the size at stocking can play an important role in the survival probability of introduced individuals. Not only because larger individuals are less prone to predation but also because of their ability to sustain poorer resource conditions than small individuals.

The DEB stand-alone model with ad libitum food but with a seasonal temperature regime predicts that the harvestable length is reached at an age of 6 . The population dynamical model predicts a similar age at which a harvestable size is reached. This is despite the fact that in the stand-alone version growth is limited only by the intake rate of lobsters while in the population dynamical model food availability plays a role. Not only is there a maximum in food density in the form of a given carrying capacity, there is also the seasonal effect of temperature causing a reduction in resource productivity at lower temperatures. The population dynamical model results suggest that this seasonality in resource productivity has little influence on growth. Stocking at a size of $5 \mathrm{~cm}$ CL would imply that this size is reached after 3 years. Stocking at smaller sizes will result in a longer period before harvestable sizes are reached, e.g. stocking at $\sim 2 \mathrm{~cm} \mathrm{CL}$ will result in a 5 year period. The seasonality in physiological rates due to changes in temperature are hence more limiting to growth than the seasonal reduction in resource productivity. Adding resources will only enhance lobster growth rate provided that resource productivity is limited and not the intake rate. Whether resource productivity is the most important limiting factor in field conditions will depend on ambient temperatures which in case are likely to show high inter annual variability and lobster size. Resource enhancement can aid growth rates when multiple individuals compete for resources and thereby reduce resource density to such levels that growth is hampered.

The surface area of a monopile provides food for a single lobster to reach the harvestable size based on the current parameterization. A lack of records on naturally occurring densities of (large) European lobster does not confirm nor reject this finding. Bouma and Lengkeek (2012) did not report the presence of lobsters in the wind park surveyed, 4 years after the park became operational. The reason for absence of lobster in unknown.

The current model does not take refuge holes into account. Such an addition might be useful if field observations demonstrate that lobster densities are indeed limited by shelter on the monopiles rather than food conditions. In such a case the population model can easily be adapted to include density regulation based on shelter availability. 


\section{Conclusions, discussion and recommendations}

This desk study describes the biology of the European lobster H. gammarus. Using the derived data a model was developed to describe the growth of the European lobster under assumed conditions on the anti-scouring of monopiles in Dutch OWFs. One of the main questions to answer was if, theoretically, local productivity supports the continuous harvesting of lobsters with passive fishery methods.

\subsection{Main findings}

\subsubsection{Lobster ecology}

As a first finding lobster is characterised as a large top end predator. It exhibits a k-strategy life style, able to reach very old ages (e.g. an estimated 72 years). The planktonic and early benthic stages are very vulnerable and susceptible to predation. It grows slowly, taking at least four years to reach maturity in favourable conditions although in general maturity is thought to start from five years old depending on ambient conditions. Reproduction occurs in a two to three year cycle. The eggs are held on the pleopods for approximately a year until hatching. In itself the reproduction is sufficient to maintain populations at high levels (given the high age). However, global lobster populations have suffered considerable fishing pressure and stock collapse only recovering slowly now at some regions partly due to appropriate management (the MCRS and stock enhancement programmes, Wickins \& Lee, 2002, Prodöhl et al., 2006, Phillips, 2013).

In principle European lobsters have a high enough mobility to colonize the entire North Sea as one genetic population using wrecks, hard substrates etcetera as stepping stones (Krone and Schröder, 2011). Still unexpected differentiated genetic clusters occur which should have been fully connected and exchanging based on this mobility. Apparently the exchange is less than based on theoretical estimates of mobility.

The limited exchange implies that when exploiting lobsters at OWFs an exploitation management plan should be established based on the local OWF population dynamics and the presence of lobsters nearby on the soft sediments. Also stock enhancement using larvae or juveniles from local populations might be an additional option. The seafloor at the OWF could be an issue in choosing at which size to release juvenile lobsters since the earliest benthic stages can dig their holes. When the seafloor is more sandy than solid mud, early stages are entirely depending on the crevices between stones and cobbles. In addition they actively harvest the walls of their residence. The more productive the seafloor bottom is (in terms of worms, amphipods etc.) the less frequent these vulnerable stages have to expose themselves to predation and currents (Lawton \& Lavalli, 1995, Jensen et al., 2000, Wickins $\&$ Lee, 2002). In this sense older and larger stages ( $>$ an estimated $15 \mathrm{~mm} \mathrm{CL}$ ) are less dependent on bottom type (and more on availability of crevices).

All sizes European lobster prefer hard substrate with crevices to hide. At younger stages they are entirely dependent on shelter in order to evade predation. At later stages they become less dependent on shelter but keep a preference for hard substrate. Crevices should have the size to accommodate the complete body and for all sizes. Younger animals of European and American lobsters move to larger crevices while growing (Lawton \& Lavalli, 1995, Jensen et al., 2000, Dunnington et al., 2005, Phillips, 2013)

Lobster densities at suitable substrates of a fished populations are 1 lobster per $\geq 150 \mathrm{~m}^{2}$ (four observations). Two extremely high densities were found for European lobster at 1 lobster per $\sim 4-6 \mathrm{~m}^{2}$. American lobsters can have on average much higher natural densities ( 1 lobster every $6 \mathrm{~m}^{2}$ ) but one 
should keep in mind that this species is migratory (Dunnington et al., 2005, Geraldi et al., 2009, Phillips, 2013) that might explain that a given substrate does not have to provide food year round. In addition, they have a higher mobility so likely have a greater foraging area .

\subsubsection{DEB modelling and lobster production}

The DEB model predicts lobster growth in line with growth reported in literature although length at age from field data shows a large variability. Based on the current model parameter setting resource production is limiting lobster growth and abundance while ensuring that lobsters reach marketable sizes. The current model setting predicts that 1 lobster can reach marketable size after 3 years, provided a stocking length at $50 \mathrm{~mm} \mathrm{CL}$. Model resource productivity was based on mussel density estimated at a limited survey of monopiles in a Dutch OWF. However, resource production might differ between monopiles, parks and temperature regimes, and higher productivity will support more lobsters and/or have a positive effect on individual growth rates.

The population model does not take recruitment into account, the reason being that a single monopile surface area is being studied and that little is known on factors determining recruitment success in wild populations, nor the geographical extent of such populations in the North Sea. Energy losses through egg production is taken into account. In addition, no lobsters were observed at a studied wind park 4 years after operation started (Bouma and Lengkeek, 2012). This suggests that at least the chance of natural recruitment on monopiles in wind parks in the North Sea is low. However, when the expectancy is 1 lobster per monopile, changes of encounter during a scientific dive are not that high.

The modelled European lobster productivity is in line with low densities found in the field, using the OWF hard substrate and anti-scouring data from Bouma \& Lengkeek (2012). One lobster could be supported by one monopile given its anti-scouring surface ( 1 per $\left.364 \mathrm{~m}^{2}\right)$.

\subsubsection{Enhancement strategies}

The current literature survey on lobster ecology linked observations to the proposed enhancement strategies (habitat, stock and food). All strategies seem to have a potential added value to lobster production. As a basic requirement, monitoring is needed to establish how much and which sizeclasses of $H$. gammarus are present in the OWFs to think on any strategy at all.

Habitat enhancement can serve to increase the amount of crevices available. Taking the expected crevice size into account for OWF Prinses Amalia and OWF Luchterduinen (maximally $8 \times 8 \times 8 \mathrm{~cm}$, Table 7), theoretically there could be a lack of suitable crevices for larger sized lobster in the OWFs preselected for TKI Win-Wind. Adding extra habitat by means of hard substrate with crevices might stimulate the presence of legal catchable lobster (at $85 \mathrm{~mm} \mathrm{CL}$, an estimated $240 \mathrm{~mm}$ total length, Prodhöl et al., 2006). A potential new habitat should be carefully designed accommodating several sizes classes of lobster, having sufficient water and oxygen refreshment and also enabling stocks of the typical food like mussels and crabs. In addition, extra hard substrate can serve as fundament for extra biota and thereby food.

Stock enhancement has a potential to help to surpass the vulnerable pelagic and early settlement stages (up to $\sim 15 \mathrm{~mm} \mathrm{CL}$ or even larger). It can also safeguard supply of animals since natural recruitment is low. Not all OWFs have European lobster whereas one would expect presence. Also Figure 8 shows e.g. remarkable patterns of suitability of wrecks: wrecks nearby each other have marked differences in suitability. These phenomena can reflect differences in habitat suitability or supply of larvae.

Food seems the most obvious limiting factor. Food enhancement (by e.g. improving quality by seeding or improving availability by depositing discards or extra habitat) can help to increase productivity and populations. 


\subsection{Recommendations}

\subsubsection{Early stages}

In literature major uncertainties exist on the larval and early settlement stages in terms of abundance, transport, settlement and survival, especially the early settlement stages (Phillips, 2013). One wonders whether to invest in research on early life stages in the case of commercially exploiting Dutch OWFs for lobsters. Especially given the low rates of colonisation (e.g. Krone and Schröder, 2011) and the vulnerability of the early life stages, difficulties in natural recruitment might be surpassed by active restocking (in the setting of exploitation).

\subsubsection{Presence, density and habitat}

It is unclear whether European lobsters are present in OWFs. Bouma and Lengkeek (2012) did not encounter any during a limited diving survey (only four diving days, diving on neap tide, with two poor visibility days on three monopiles). The TKI Energy project WinWind will pay attention to the presence, densities and population size of lobster in OWF Prinses Amalia and/ or OWF Luchterduinen. Also presence and densities of lobster on the surrounding substrates needs to be assessed in order to determine the potential of colonisation by larger lobsters.

In general densities are a rarely assessed aspect of European lobsters ecology (Skerrit, 2014). More data are needed to get more insight in the quantitative biology of $H$. gammarus. It is advisable to include length measurements, characterisation of the substrate, food availability and measurements of (a)biotic factors like temperature ranges, depth etc. (see Table 3).

In this sense Table 3 is striking due to its incongruence. It should be noted that the data compiled by Kristiansen et al. (2004) are from lobster aquacultures of both $H$. gammarus and $H$. americanus. The data of https://eol.org/pages/46505673/data are field data. This data base should be extended with measurements of Dutch circumstances especially in OWFs.

\subsubsection{Position and functioning in the food web}

More information is needed on the position and functioning of $H$. gammarus in the food web. What are the predators on European lobster in different life stages and what is $H$. gammarus preying upon in the Dutch NCP and OWFs specifically? Information is needed on stomach contents of both European lobsters of different sizes and their potential predators. Also the composition of the food web and trophic layers needs to be studied with and without European lobster in order to determine the potential (mutual) influences.

\subsubsection{Physiology and DEB modelling}

There is a lack of physiological data in literature. It is advisable to do some basic studies on the physiology of this species. A series of experiments could aid a parameterization of the DEB model for lobster specifically for North Sea conditions.

\subsubsection{Primary production and benthic-pelagic coupling}

Initially it was anticipated to start the productivity modelling at the trophic level of PP. However, both the PP and the benthic-pelagic coupling are scarcely investigated. The literature was not that detailed that trustworthy parameters and variables could be derived for a reliable productivity estimate at the tertiary production level of European lobster. This urges for more research on the benthic-pelagic and local processes driving secondary production in relation to PP and monopile characteristics.

\subsubsection{Enhancement strategies}

The literature survey on the enhancement strategies has underpinned the importance of enhancement to promote lobsters. Current results suggest that improvement of habitat in quantity and quality, in 
order to have more and larger crevices and more food, are more useful to promote commercial exploitation. In the TKI Energy project Win-Wind more detailed information will be generated on the three strategies in order to get more insights in what enhancement strategy to adopt under which conditions. An important input for which strategy is most suitable will be the absence or presence of lobsters (number and size distribution) encountered. Information on food availability as well as crevice (size and numbers) needs to be gathered. The outcome of such surveys will determine the economic potential and also the urgency to adopt one or several enhancement strategies.

More research is needed in order to determine habitat suitability and differentiating factors on the Dutch NCP. Is that e.g. the suitability of the habitat (crevice numbers and characteristics), or is that food availability. It might even be supply of animals by recruitment by larvae or immigration by adults. Although the mobility of both should be enough and not be a limiting factor (see next sections). 


\section{Quality Assurance}

Wageningen Marine Research utilises an ISO 9001:2015 certified quality management system. This certificate is valid until 15 December 2021. The organisation has been certified since 27 February 2001. The certification was issued by DNV GL.

Furthermore, the chemical laboratory at IJmuiden has NEN-EN-ISO/IEC 17025:2005 accreditation for test laboratories with number L097. This accreditation is valid until $1^{\text {th }}$ of April 2021 and was first issued on 27 March 1997. Accreditation was granted by the Council for Accreditation. The chemical laboratory at IJmuiden has thus demonstrated its ability to provide valid results according a technically competent manner and to work according to the ISO 17025 standard. The scope (L097) of de accredited analytical methods can be found at the website of the Council for Accreditation (www.rva.nl).

On the basis of this accreditation, the quality characteristic $Q$ is awarded to the results of those components which are incorporated in the scope, provided they comply with all quality requirements. The quality characteristic $\mathrm{Q}$ is stated in the tables with the results. If, the quality characteristic $\mathrm{Q}$ is not mentioned, the reason why is explained.

The quality of the test methods is ensured in various ways. The accuracy of the analysis is regularly assessed by participation in inter-laboratory performance studies including those organized by QUASIMEME. If no inter-laboratory study is available, a second-level control is performed. In addition, a first-level control is performed for each series of measurements.

In addition to the line controls the following general quality controls are carried out:

- Blank research.

- Recovery.

- Internal standard

- Injection standard.

- Sensitivity.

The above controls are described in Wageningen Marine Research working instruction ISW 2.10.2.105. If desired, information regarding the performance characteristics of the analytical methods is available at the chemical laboratory at IJmuiden.

If the quality cannot be guaranteed, appropriate measures are taken. 


\section{References}

Agnalt, A. L., E. Farestveit, K. Gundersen, K. E. Jørstad and T. S. Kristiansen (2009). "Population characteristics of the world's northernmost stocks of European lobster (Homarus gammarus) in Tysfjord and Nordfolda, northern Norway." New Zealand Journal of Marine and Freshwater Research 43(1): 47-57.

Agnalt, A.-L., E. S. Grefsrud, E. Farestveit and K. E. Jørstad (2017). "Training camp-A way to improve survival in European lobster juveniles?" Fisheries Research 186: 531-537.

Alvarez-Fernandez, S. and R. Riegman (2014). "Chlorophyll in North Sea coastal and offshore waters does not reflect long term trends of phytoplankton biomass." Journal of Sea Research 91: 35-44.

Bannister R. C. A. and J. T. Addison (1998). Enhancing lobster stocks: a review of recent European methods, results, and future prospects. Bulletin of Marine Science 62(2): 369-387.

Bannister, R. C. A., J. T. Addison and S. R. J. Lovewell (1994). "Growth, Movement, Recapture Rate and Survival of Hatchery-Reared Lobsters (Homarus gammarus (Linnaeus, 1758)) Released into the Wild on the English East Coast." Crustaceana 67(2): 156-172.

Bell, M. C., D. R. Eaton, R. Bannister and J. Addison (2003). A mark-recapture approach to estimating population density from continuous trapping data: Application to edible crabs, Cancer pagurus, on the east coast of England.

Boudreau, S. A. and B. Worm (2010). "Top-down control of lobster in the Gulf of Maine: insights from local ecological knowledge and research surveys." Marine Ecology Progress Series 403: 181-191.

Bouma, S. and W. Lengkeek (2012). Benthic communities on hard substrates of the offshore wind farm Egmond aan Zee (OWEZ), Bureau Waardenburg.

Brey, T., H. Rumohr and S. Ankar (1988). "Energy content of macrobenthic invertebrates: general conversion factors from weight to energy. ." Journal of Experimental Marine Biology and Ecology 117: 271-278.

Brummelhuis, E. B. M., M. v. Asch, D. v. d. Ende, K. Troost and C. v. Zweeden (2013). Japanse oesterbanken op droogvallende platen in de Nederlandse kustwateren in 2013: bestand en arealen. IJmuiden, IMARES Wageningen UR.

Buck, B. H., G. Krause, B. Pogoda, B. Grote, L. Wever, N. Goseberg, M. F. Schupp, A. Mochtak and D. Czybulka (2017). The German Case Study: Pioneer Projects of Aquaculture-Wind Farm Multi-Uses. Aquaculture Perspective of Multi-Use Sites in the Open Ocean: The Untapped Potential for Marine Resources in the Anthropocene. B. H. Buck and R. Langan. Cham, Springer International Publishing: 253-354.

Contarini, G., N. Perrella, J. Hickey and R. Ballestrazzi (2008). "Hatchery production of European lobster (Homarus gammarus, L.): broodstock management and effects of different holding systems on larval survival." Italian Journal of Animal Science 7(3): 351-362.

de Roos, A. M., O. Dieckmann and J. A. J. Metz (1992). "Studying the dynamics of structured population models: A versatile technique and its application to Daphnia." The American Naturalist 139(1): 123-147.

Duffill-Telsnig, J. (2014). The feasibility of aquaculture, aquaponics and a lobster hatchery., Amble Development Trust Report: 33.

Duffill-Telsnig, J. (2014). The feasibility of aquaculture, aquaponics and a lobster hatchery., Amble Development Trust Report: 33.

Dunnington, M. J., R. A. Wahle, M. C. Bell and N. R. Geraldi (2005). "Evaluating local population dynamics of the American lobster, Homarus americanus, with trap-based mark-recapture methods and seabed mapping." New Zealand Journal of Marine and Freshwater Research 39(6): 1253-1276.

Ebenhöh, W., C. Kohlmeier and P. J. Radford (1995). "The benthic biological submodel in the European regional seas ecosystem model." Netherlands Journal of Sea Research 33(3): 423-452.

Ellis, C. D., D. J. Hodgson, C. L. Daniels, M. Collins and A. G. F. Griffiths (2017). "Population genetic structure in European lobsters: implications for connectivity, diversity and hatchery stocking." Marine Ecology Progress Series 563: 123-137. 
Elner, R. and A. Campbell (1987). "Natural diets of lobster Homarus americanus from barren ground and macroalgal habitats off southwestern Nova Scotia, Canada." Mar. Ecol. Prog. Ser. 37: 131140.

Factor, J. R. (1995). Biology of the Lobster. San Diego, Academic Press.

Fogarty, M. J. and J. T. Addison (1997). "Modelling capture processes in individual traps: entry, escapement and soak time." ICES Journal of Marine Science 54(2): 193-205.

Forster, J., A. G. Hirst and D. Atkinson (2012). "Warming-induced reductions in body size are greater in aquatic than terrestrial species." Proceedings of the National Academy of Sciences of the United States of America 109(47): 19310-19314.

Galparsoro, I., Á. Borja, J. Bald, P. Liria and G. Chust (2009). "Predicting suitable habitat for the European lobster (Homarus gammarus), on the Basque continental shelf (Bay of Biscay), using Ecological-Niche Factor Analysis." Ecological Modelling 220(4): 556-567.

Geraldi, N. R., R. A. Wahle and M. Dunnington (2009). "Habitat effects on American lobster (Homarus americanus) movement and density: insights from georeferenced trap arrays, seabed mapping, and tagging." Canadian Journal of Fisheries and Aquatic Sciences 66(3): 460-470.

Griffiths, J. R., M. Kadin, F. J. A. Nascimento, T. Tamelander, A. Tornroos, S. Bonaglia, E. Bonsdorff, V. Bruchert, A. Gardmark, M. Jarnstrom, J. Kotta, M. Lindegren, M. C. Nordstrom, A. Norkko, J. Olsson, B. Weigel, R. Zydelis, T. Blenckner, S. Niiranen and M. Winder (2017). "The importance of benthic-pelagic coupling for marine ecosystem functioning in a changing world." Global Change Biology 23(6): 2179-2196.

Hanson, J. M. (2009). "Predator-prey interactions of American lobster (Homarus americanus) in the southern Gulf of St. Lawrence, Canada." New Zealand Journal of Marine and Freshwater Research 43(1): 69-88.

Hepper, B. T. (1967). "On the Growth at Moulting of Lobsters (Homarus Vulgaris) in Cornwall and Yorkshire." Journal of the Marine Biological Association of the United Kingdom 47(3): 629-643.

Hepper, B. T. (1978). Population dynamics of the lobster Homarus gammarus (L.) off the coasts of England. . Fish. Res. tech. Rep. M. A. F. F. Dir. fish. Res., Lowestoft. 41.

Herman, A. W., M. R. Mitchell and S. W. Young (1984). "A continuous pump sampler for profiling copepods and chlorophyll in the upper oceanic layers." Deep Sea Research Part A. Oceanographic Research Papers 31(4): 439-450.

Holthuis, L. B. (1991). FAO Species Catalogue. Marine lobsters of the World. An annotated and illustrated catalogue of species of interest to fisheries known to date. FAO Fisheries Synopsis, FAO 125: 13 .

Hooper, T. and M. Austen (2014). "The co-location of offshore windfarms and decapod fisheries in the UK: Constraints and opportunities." Marine Policy 43: 295-300.

Howard, A. E. (1988). "Lobster behaviour, population structure and enhancement." Symp. zool. Soc. Lond. 59: 355-364.

Hoydalsvik, M. (2017). Effects of marine protected areas on European lobster (Homarus gammarus) in Skagerrak, Norway. MSc.

Jensen, A., J. Wickins and C. Bannister (2000). The potential use of artificial reefs to enhance lobster habitat. Artificial reefs in European seas. A. C. Jensen, K. J. Collins and L. A.M.P. Great Britain, Kluwer Academic Publishers: 379-401.

Jensen, A C., K.J. Collins, E.K. Free and R.CA. Bannister (1994). "Lobster (Hamarus gammarus) movement on an artificial reef; the potential use of artificial reef for stock enhancement. . ." Crustaceana 67(2): 198-211.

Joschko, T. J., B. H. Buck, L. Gutow and A. Schröder (2008). "Colonization of an artificial hard substrate by Mytilus edulis in the German Bight." Marine Biology Research 4(5): 350-360.

Kooijman, S. A. L. M. (2009). Dynamic Energy Budgets Theory for Metabolic Organisation. Cambridge, Cambridge University Press

Kooijman, S. A. L. M. and S. Augustine. (2017). "AmP Homarus gammarus, version 2017/08/24.

Kristiansen, T. S., A. Drengstig, A. Bergheim, T. Drengstig, I. Kollsgard, R. Svendsen, E. Nostvold, E. Farestveit and L. Aardal (2004). Development of methods for intensive farming of European lobster in recirculated seawater. Results from experiments conducted at Kvitsoy lobster hatchery from 2000 to 2004, Havforskningsinstituttet - Institute of Marine Research.

Kröncke, I. (2006). "Structure and function of macrofaunal communities influenced by hydrodynamically controlled food availability in the Wadden Sea, the open North Sea, and the Deep-sea. A synopsis. ." Senckenbergiana Maritima 36: 123-164. 
Krone, R. and A. Schröder (2011). "Wrecks as artificial lobster habitats in the German Bight." Helgoland Marine Research 65(1): 11-16.

van den Bogaart L., Poelman M., Neitzel S., Tonk L., van der Wal J.T., Coolen J., Machiels M. met bijdrage van MJC Rozemeijer, S. Vergouwen, L. van Duren (2019). Geschiktheid zeewindparken voor maricultuur en passieve visserij. Een kwalitatieve beoordeling van geschiktheid van windparklocaties voor voedselproductie. WUR, Wageningen Marine Research Report BO-43-023.03005 Concept.

Laurans, M., S. Fifas, S. Demaneche, S. Brérette and O. Debec (2009). "Modelling seasonal and annual variation in size at functional maturity in the European lobster (Homarus gammarus) from self-sampling data." ICES Journal of Marine Science 66(9): 1892-1898.

Lawton, P. and K. L. Lavalli (1995). Chapter 4 - Postlarval, Juvenile, Adolescent, and Adult Ecology. Biology of the Lobster. J. R. Factor. San Diego, Academic Press: 47-88.

Lees, K. J., A. C. Mill, D. J. Skerritt, P. A. Robertson and C. Fitzsimmons (2018). "Movement patterns of a commercially important, free-ranging marine invertebrate in the vicinity of a bait source." Animal Biotelemetry 6(1): 8.

Leewis, R. L., d. V. I., B. H.C., d. K. M. and v. M. G.W.N.M. (1997). Kunstriffen in Nederland. Rijkswaterstaat RIKZ. WB360-13 ZH.

Lengkeek, W., K. Didderen, M. Teunis, F. Driessen, J. W. P. Coolen, O. G. Bos, S. A. Vergouwen, T. Raaijmakers, M. B. de Vries and M. van Koningsveld (2017). Eco-friendly design of scour protection: potential enhancement of ecological functioning in offshore wind farms: Towards an implementation guide and experimental set-up. Bureau Waardenburg Report. 17-001.

Linley E.A.S., T.A. Wilding, K. Black, A.J.S. Hawkins and S. Mangi (2008). Review of the reef effects of offshore wind farm structures and their potential for enhancement and mitigation. Report from PML Applications Ltd and the Scottish Association for Marine Science to the Department for Business, Enterprise and Regulatory Reform (BERR). Contract No: RFCA/005/0029P.

Lipscius, R. N., D. B. Eggleston, F. J. Fodrie, J. van der Meer, K. A. Rose, R. P. Vasconcelos and K. E. van de Wolfshaar (Under Review). "Modeling quantitative value of coastal habitatsfor exploited species."

Lizárraga-Cubedo, H. A., I. Tuck, N. Bailey, G. J. Pierce and J. A. M. Kinnear (2003). "Comparisons of size at maturity and fecundity of two Scottish populations of the European lobster, Homarus gammarus." Fisheries Research 65(1): 137-152.

Mercer, J. P. R. C. A. B., G. I. v. d. Meeren, D. M. V. Debuse, R. B. S. Lovewell, A. Linnane and B. Ball (2001). "An overview of the LEAR (Lobster Ecology and Recruitment) project: the results of field and experimental studies on the juvenile ecology of Homarus gammarus in cobble." Marine and Freshwater Research 52:: 1291-1302.

Miller, R. J., K. H. Mann and D. J. Scarratt (1971). "Production Potential of a Seaweed-Lobster Community in Eastern Canada." Journal of the Fisheries Research Board of Canada 28(11): 17331738.

Pandian, T. J. (1970). "Ecophysiological studies on the developing eggs and embryos of the european lobster Homarus gammarus. ." Marine Biology 5: 154--167.

Pandian, T. J. (1970). "Ecophysiological studies on the developing eggs and embryos of the European lobster Hommarus gammarus." Marine Biology 5: 154-167.

Phillips B. F. (2013). Lobsters: biology, management, aquaculture and fisheries, John Wiley \& Sons Ltd.

Prodöhl, P. A., K. E. Jørstad, A. Triantafyllidis, V. Katsares and C. Triantaphyllidis (2006). European lobster-Homarus gammarus. Genetic Impact of Aquaculture Activities on Native Populations. Final Scientific Report: 91-98.

Roach, M., M. Cohen, R. Forster, A. S. Revill and M. Johnson (2018). "The effects of temporary exclusion of activity due to wind farm construction on a lobster (Homarus gammarus) fishery suggests a potential management approach." Ices Journal of Marine Science 75(4): 1416-1426.

Rozemeijer, M. J. C., D. Slijkerman, O. G. Bos, C. Röckmann, A. J. Paijmans and P. Kamermans (2017). Bouwen met Noordzee-natuur : uitwerking Gebiedsagenda Noordzee 2050. IJmuiden, Wageningen Marine Research.

Sainte-Marie, B. and D. Chabot (2002). "Ontogenetic shifts in natural diet during benthic stages of American lobster (Homarus americanus), off the Magdalen Islands." 100: 110-116.

Scarratt, D. J. (1973). Lobster populations on a man made rocky reef. Copenhagen, ICES.

Schellekens, T. and R. Witbaard (2012). DEB_ensis vs. data, IMARES. 
Schmalenbach I. (2009). Studies on the developmental conditions of the European lobster (Homarus Gammarus Linnaeus, 1758) at the rocky island of Helgoland (German Bight, North Sea) PhD.

Schmalenbach, I., F. Mehrtens, M. Janke and F. Bucholz, (2011). A mark-recapture study of hatcheryreared juvenile European lobsters, Homarus gammarus, released at the rocky island of Helgoland (German Bight, North Sea) from 2000 to 2009.Fish. Res. 108, 22-30.

Schmalenbach, I., F. Mehrtens, M. Janke and F. Buchholz (2011). Landings of European lobster (Homarus gammarus) and edible crab (Cancer pagurus) from 1615 to 2009, Helgoland, North Sea. Supplement to: Schmalenbach, I et al. (2011): A mark-recapture study of hatchery-reared juvenile European lobsters, Homarus gammarus, released at the rocky island of Helgoland (German Bight, North Sea) from 2000 to 2009. Fisheries Research, 108(1), 22-30, https://doi.org/10.1016/j.fishres.2010.11.016, PANGAEA.

Schuiling, E. and A. C. Smaal (1998). Het zoet in de pap. Een literatuurstudie naar de effecten van verhoogde zoetwatertoevoer op commercieel belangrijke soorten in de Oosterschelde IJmuiden, RIVO-DLO: 47.

Seitz, R. D., H. Wennhage, U. Bergström, R. N. Lipcius and T. Ysebaert (2014). "Ecological value of coastal habitats for commercially and ecologically important species." ICES Journal of Marine Science 71(3): 648-665.

Sheehy, D. J. (1976). "Utilization of Artificial Shelters by the American Lobster (Homarus americanus)." Journal of the Fisheries Research Board of Canada 33(7): 1615-1622.

Sheehy, M. R. J. and R. C. A. Bannister (2002). "Year-class detection reveals climatic modulation of settlement strength in the European lobster, Homarus gammarus." Canadian Journal of Fisheries and Aquatic Sciences 59(7): 1132-1143.

Sheehy, M. R. J., R. C. A. Bannister, J. F. Wickins and P. M. J. Shelton (1999). "New perspectives on the growth and longevity of the European lobster (Homarus gammarus)." Canadian Journal of Fisheries and Aquatic Sciences 56(10): 1904-1915.

Skerritt, D. J. (2014). Abundance, interaction and movement in a European Lobster Stock. PhD, Newcastle University.

Skerritt, D. J., C. Fitzsimmons, N. V. C. Polunin, P. Berney and M. H. Hardy (2012). Investigating the impact of offshore wind farms on European Lobster (Homarus gammarus) and brown Crab (Cancer pagurus) fisheries. Technical Report.

Skerritt, D. J., P. A. Robertson, A. C. Mill, N. V. C. Polunin and C. Fitzsimmons (2015). "Fine-scale movement, activity patterns and home-ranges of European lobster Homarus gammarus." Marine Ecology Progress Series 536: 203-219.

Smaal, A., P. Kamermans, F. Kleissen, L. van Duren and T. van der Have (2017). Flat oysters on offshore wind farms : opportunities for the development of flat oyster populations on existing and planned wind farms in the Dutch section of the North Sea. Yerseke, Wageningen Marine Research.

Smith, I. P., A. C. Jensen, K. J. Collins and E. L. Mattey (2001). "Movement of wild European lobsters Homarus gammarus in natural habitat." Marine Ecology Progress Series 222: 177-186.

Sørdalen, T. K., K. T. Halvorsen, H. B. Harrison, C. D. Ellis, L. A. Vøllestad, H. Knutsen, E. Moland and E. M. Olsen (2018). "Harvesting changes mating behaviour in European lobster." Evolutionary Applications 11(6): 963-977.

Steenbergen, J., T. v. Kooten, K. E. van de Wolfshaar, B. K. Trapman and K. J. v. d. Reijden (2015). Management options for brown shrimp (Crangon crangon) fisheries in the North Sea. IJmuiden, IMARES.

Tangelder, M., P. C. Goudswaard and Y. v. Es (2015). Bepaling zware metalen in kreeften op nieuwe vooroevers in de Oosterschelde. IJmuiden, IMARES Wageningen UR.

Timmermann, K., J. Norkko, U. Janas, A. Norkko, B. G. Gustafsson and E. Bonsdorff (2012). "Modelling macrofaunal biomass in relation to hypoxia and nutrient loading." Journal of Marine Systems 105: 60-69.

Topham, E. and D. McMillan (2017). "Sustainable decommissioning of an offshore wind farm." Renewable Energy 102: 470-480.

Triantafyllidis, A., A. P. Apostolidis, V. Katsares, E. Kelly, J. Mercer, M. Hughes, K. E. Jørstad, A. Tsolou, R. Hynes and C. Triantaphyllidis (2005). Mitochondrial DNA variation in the European lobster ( Homarus gammarus) throughout the range.

Tunnicliffe, V., A. Metaxas, J. Le, E. Ramirez-Llodra and L. A. Levin (2018). "Strategic Environmental Goals and Objectives: Setting the basis for environmental regulation of deep seabed mining." Marine Policy. 
Turner, R. A., M. H. Hardy, J. Green and N. V. C. Polunin (2009). Defining the Northumberland Lobster Fishery', Report to the Marine and Fisheries Agency, London., Marine and Fisheries Agency, London.

Uglem, I., M. Belchier and T. Svåsand (2005). "Age Determination of European Lobsters (Homarus Gammarus L.) by Histological Quantification of Lipofuscin." Journal of Crustacean Biology 25(1): 95-99.

van de Wolfshaar, K. E., A. M. de Roos and L. Persson (2008). "Population feedback after successful invasion leads to ecological suicide in seasonal environments." Ecology 89(1): 259-268.

van der Hout, C. M., R. Witbaard, M. J. N. Bergman, G. C. A. Duineveld, M. J. C. Rozemeijer and T. Gerkema (2017). "The dynamics of suspended particulate matter (SPM) and chlorophyll-a from intratidal to annual time scales in a coastal turbidity maximum." Journal of Sea Research 127: 105118.

Van der Meer, J., J. J. Beukema and R. Dekker (2001). "Long-term variability in secondary production of an intertidal bivalve population is primarily a matter of recruitment variability." Journal of Animal Ecology 70(1): 159-169.

Van Der Meeren, G. I. (2005). "Review. Potential of ecological studies to improve survival of cultivated and released European lobsters, Homarus gammarus " New Zealand Journal of Marine and Freshwater Research 39(2): 399-424.

van Moorsel, G. W. N. M., W. H.W and v. d. H. J. (1991). Het leven op en rond scheepswrakken en andere harde substraten in de Noordzee (1986 tot en met 1990) - een synthese -, Bureau Waardenburg. rapport 91.19.

van Moorsel, G. W. N. M. and W. H.W. (2001). Kunstmatige riffen in de Noordzee. De status 9 jaar na aanleg, Bureau Waardenburg bv., Culemborg. Rapportnummer 01-071.

Van Stralen, M. R. and S. E. W. M. (2008). Effecten van sleepnetvisserij en visserij met vaste vistuigen op vogels, zeezoogdieren, migrerende vissoorten en kreeften. Deelstudie Kreeft. , Bureau MarinX: 51.

Waddy, S. L., D. E. Aiken and D. P. V. De Kleijn (1995). Chapter 10 - Control of Growth and Reproduction. Biology of the Lobster. J. R. Factor. San Diego, Academic Press: 217-266.

Wallace, N. (2015). Report Lobster, fishing effort and habitat in-teractions in the Northumberland Lobster Fishery Newcastle University MSc in International Marine Environmental Consultancy 26.

Whiteley, N. M., A. H. Alwassia and E. W. Taylor (1990). "The effect of temperature, aerial exposure and disturbance on oxygen-consumption in the lobster, Homarus-Gammarus (L)." Marine Behaviour and Physiology 17(4): 213-222.

Wickins, J. F. and T. W. Beard (1991). "Variability in size at moult among individual broods of cultured juvenile lobsters, Homarus gammarus (L.)." Aquaculture Research 22(4): 481-489.

Wickins, J. F. and L. D.O'C. (2002). Crustacean Farming - Ranching and Culture, Blackwell Science.

Witbaard, R., G. Duineveld and M. Bergman (2013). The dynamics and growth of Ensis directus in the near coastal zone of Egmond, in relation to environmental conditions in 2011-2012, NIOZ.

Wolff, W. J. and A. J. J. Sandee (1971). "Distribution and ecology of the decapoda reptantia of the estuarine area of the rivers rhine, meuse, and scheldt." Netherlands Journal of Sea Research 5(2): 197-226.

Wood, J. M. (2018). "New estimates and complications in the assessment of female functional maturity for the European lobster (Homarus gammarus) on the Yorkshire Coast (UK)." 2018 6(2): 4.

Wood, J. M. (2018). "New estimates and complications in the assessment of female functional maturity for the European lobster (Homarus gammarus) on the Yorkshire Coast (UK)." 2018 6(2): 4.

Wright J. (2018). Lobsters on the ground: improving understanding of shellfish populations on the Northumberland coast MSc in International Marine Environmental Consultancy Newcastle University. 


\section{Justification}

Report C109/18

Project Number: 4318300086

The scientific quality of this report has been peer reviewed by a colleague scientist and a member of the Management Team of Wageningen Marine Research

Approved: $\quad$ Dr.Ir. J.W.M. Wijsman

Senior Researcher

Signature:

Date:

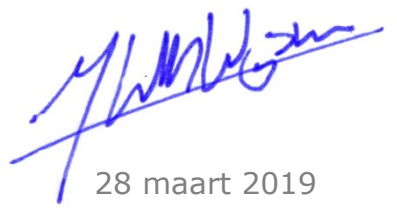

Approved:

Drs. J. Asjes

Manager integration

Signature:

Date: 


\section{Annex 1 Length definitions used for Homarus spec.}

\section{Carapace length}

Based on European Community (2006). Council Regulation (EC) No 1967/2006 of 21 December 2006, concerning management measures for the sustainable exploitation of fishery resources in the Mediterranean Sea, amending Regulation (EEC) No 2847/93 and repealing Regulation (EC) No $1626 / 94$ carapace length is measured from the rear of the eye socket to the rear of the carapace on a line parallel to the center line of the body shell. Make sure the gauge is at the extreme rear of the eye socket below the rostrum or horn. A common error is to measure from the horn located forward of the eye socket, which results in an improper measurement.

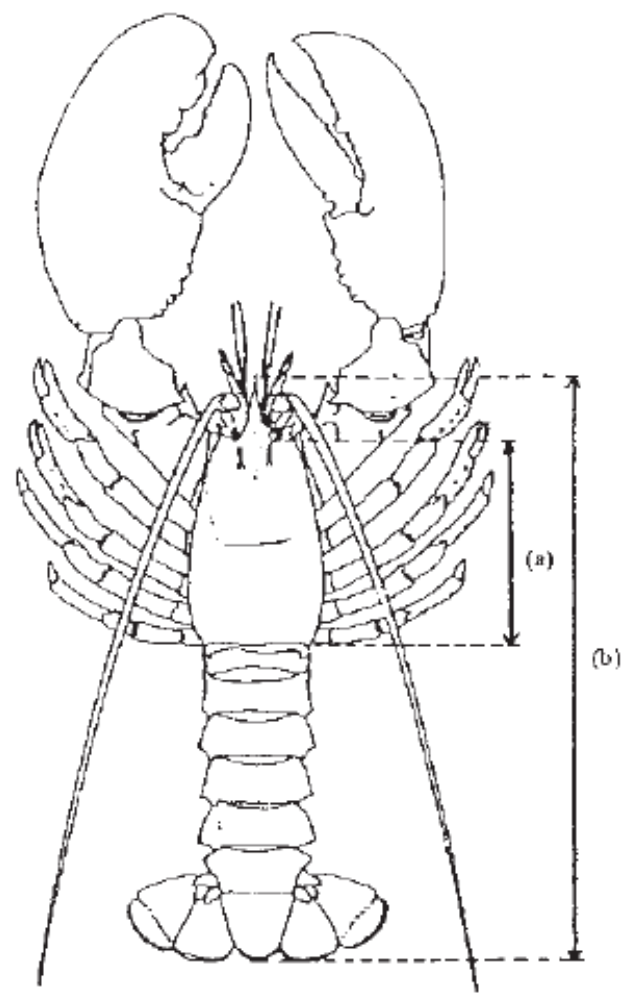

\section{(Homarus) \\ Lobster}

Figure 22 Length determination of the lobster (Homarus gammarus) by measuring (a) the length of the Carapace or (b) the total length excluding antennae and scissors (European Community, 2006). Measured lobsters have a shell length (part a of the lobster) of 83 to $87 \mathrm{~mm}$ or larger depending on country. 


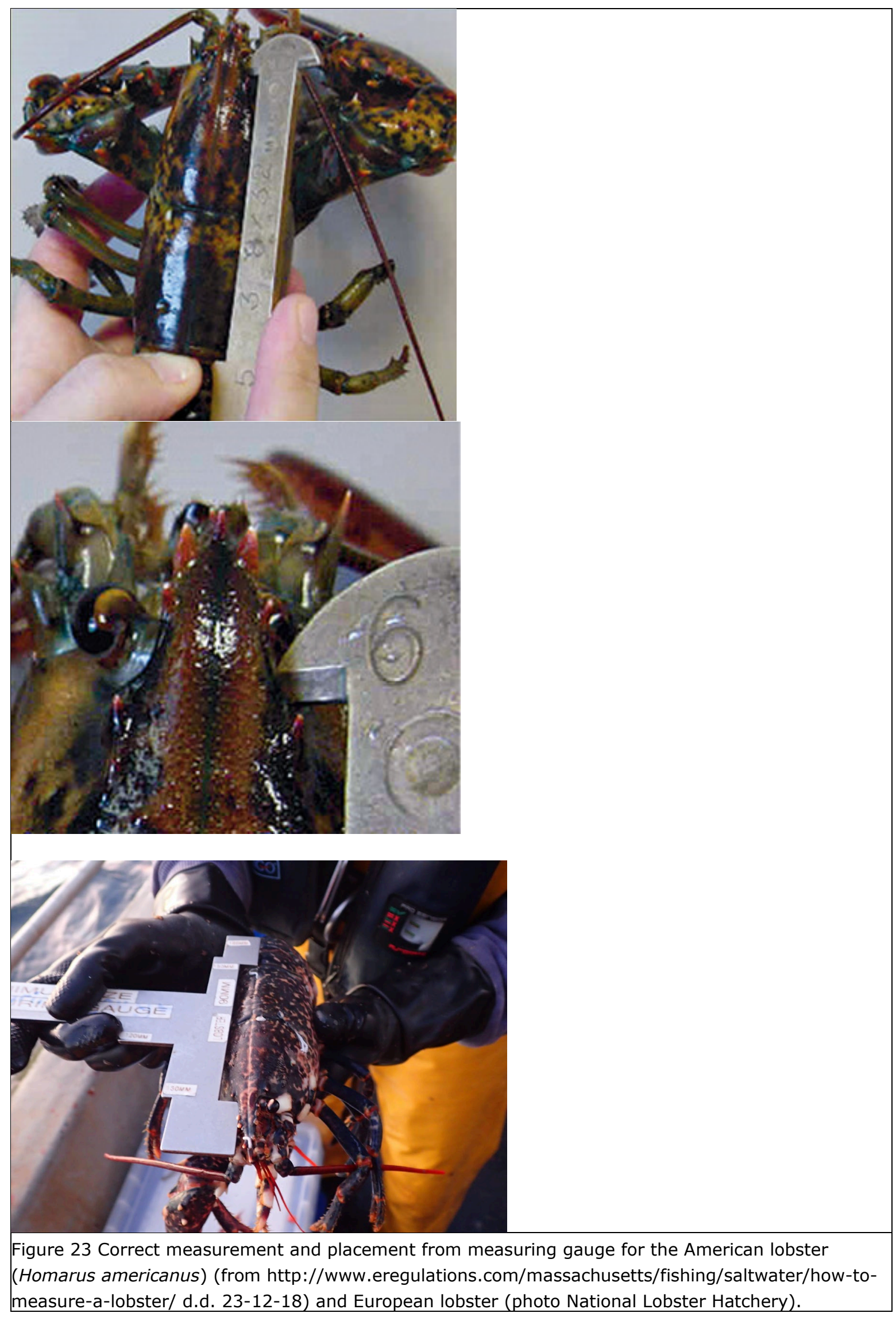

\section{Orbital Carapace Length}

As $\mathrm{CL}$; length taken from the deepest point of the orbital (Figure 23).

\section{Total length}

The total length is measured, from the tip of the rostrum to the rear end of the telson, not including the setae (Figure 22). 


\section{Annex 2 Diet composition Homarus americanus}

\begin{tabular}{|c|c|c|c|c|c|c|c|c|}
\hline & & & & & & & & \\
\hline & & & & Area-dat & e grou & & & \\
\hline Prey taxa & & $\underline{\mathbf{A}}$ & & $\underline{B}$ & & $\underline{\mathbf{C}}$ & & $\underline{\mathbf{D}}$ \\
\hline 1. Animals & 100 & $(94.2)$ & 100 & $(92.8)$ & 99 & $(97.8)$ & 100 & (97.3) \\
\hline 2. Protozoans/ foraminiferans & 1 & $(0.02)$ & 5 & $(0.21)$ & 4.5 & $(1.5)$ & 31 & $(1.6)$ \\
\hline 3. Poriferans & 1 & $(0.02)$ & 1 & $(0.19)$ & 0.5 & $(0.07)$ & & \\
\hline 4. Cnidarians & 11 & $(0.31)$ & 22 & $(0.64)$ & 15 & (039) & 21 & $(1.2)$ \\
\hline 5. Bryozoans & 1 & $(0.02)$ & - & & 1 & $(0.08)$ & - & \\
\hline 6. Molluscs & 94 & $(18.8)$ & 83 & $(21.2)$ & 90 & $(19.6)$ & 84 & $(16.5)$ \\
\hline 7. Chitons & 9 & $(0.57)$ & 34 & $(1.4)$ & 21 & $(1.6)$ & 9 & $(1.4)$ \\
\hline 8. Tonicella marmorea & - & & 2 & $(0.08)$ & 9 & $(0.88)$ & 3 & $(0.59)$ \\
\hline 9. Gastropods & 78 & $\left(\begin{array}{ll}6 & 2\end{array}\right)$ & 44 & $(2.2)$ & 10 & $(0.51)$ & 4 & $(0.15)$ \\
\hline 10. Acmae testudinalis & 2 & $(0.16)$ & 1 & $(0.02)$ & 2 & $(0.04)$ & - & \\
\hline 11. Lacuna vincta & 26 & $(18)$ & 6 & $(0.11)$ & 1 & $(0.02)$ & - & \\
\hline 12. Skeneopsis striatum & 2 & $(0.16)$ & - & & 1 & $(0.02)$ & - & \\
\hline 13. Crucibulum striatum & 1 & $(0.04)$ & - & & - & & - & \\
\hline 14. Crepidula sp. & 2 & $(0.05)$ & - & & - & & - & \\
\hline 15. Lunatia sp. & 2 & $(0.16)$ & - & & - & & - & \\
\hline 16. Mitrella sp. & 5 & $(0.26)$ & - & & - & & - & \\
\hline 17. Bivalves & 63 & $(11.8)$ & 71 & $(17.3)$ & 87 & $(16.1)$ & 78 & $(14.0)$ \\
\hline $\begin{array}{l}\text { 18. Mussels (Mytilus edulis, Modiolus } \\
\text { modiolus) }\end{array}$ & 49 & $(9.6)$ & 62 & $(16.2)$ & 85 & $(15.0)$ & 77 & $(13.8)$ \\
\hline 19. Cerastoderma pinnulatum & 10 & $(0.57)$ & 2 & $(0.03)$ & 0.3 & $(0.01)$ & - & \\
\hline 20. Anomia sp. & 4 & $(0.27)$ & 4 & $(0.08)$ & - & & - & \\
\hline 21. Hiatella sp. & - & & 4 & $(0.22)$ & 1 & $(0.01)$ & - & \\
\hline 22. Mya sp. & - & & 1 & $(0.02)$ & - & & - & \\
\hline 23. Musculus sp. & 6 & $(0.26)$ & 2 & $(0.03)$ & - & & - & \\
\hline 24. Polychaetes & 54 & $(4.0)$ & 48 & $(2.8)$ & 50 & $(5.7)$ & 36 & $(5.6)$ \\
\hline 25. Nereis $\mathrm{sp}$. & 37 & $(1.37)$ & 37 & $(0.96)$ & 22 & $(2.4)$ & 21 & (3.0) \\
\hline 26. Polynoids & 23 & $(1.1)$ & 11 & $(1.4)$ & 24 & (2.1) & 8 & $(1.5)$ \\
\hline 27. Spirorbis sp . & 2 & $(0.05)$ & - & & - & & - & \\
\hline 28. Cistena sp. & 3 & $(1.3)$ & 2 & $(0.34)$ & 0.5 & $(0.02)$ & - & \\
\hline 29. Crustaceans & 86 & $(28.2)$ & 79 & (29.1) & 41 & $(15.5)$ & 31 & (7.5) \\
\hline 30. Barnacles & 3 & $(0.09)$ & 2 & $(0.03)$ & - & & - & \\
\hline 31. Isopods & 11 & $(1.2)$ & 1 & $(0.02)$ & 0.3 & $(0.01)$ & - & \\
\hline 32. Idotea sp. & 3 & $(0.57)$ & - & & - & & - & \\
\hline 33. Amphipods & 23 & $(1.2)$ & 3 & $(006)$ & 2 & (008) & 0.3 & $(0.03)$ \\
\hline 34. Corophium & 1 & $(0.02)$ & 1 & $\left(\begin{array}{lll}0 & 02\end{array}\right)$ & - & & - & \\
\hline 35. Ampithoe rubricata & 10 & $(0.73)$ & - & & - & & - & \\
\hline 36. Decapods & ?6 & $(25)$ & 74 & (2A.1I & 36 & -15.2 & 28 & $(7.2)$ \\
\hline
\end{tabular}




\begin{tabular}{|l|r|r|r|r|r|r|r|c|} 
37. Homarus americanus & 16 & $(6.2)$ & 16 & $(6.1)$ & 19 & $(11.9)$ & 2 & $(0.51)$ \\
\hline 38. Pagurus sp. & 26 & $6.7)$ & 11 & $(1.8)$ & - & & - & \\
\hline 39. Cancer sp. & 30 & $(9.5)$ & 37 & $(14.9)$ & 2 & $(0.53)$ & 1 & $(0.49)$ \\
\hline 40. Hyas sp. & - & & 3 & $(1.5)$ & 4 & $(0.76)$ & 2 & $(0.78)$ \\
\hline 41. Carcinus maenas & 2 & $(0.18)$ & - & & - & & - & \\
\hline 42. Caridion sp. & 1 & $(0.02)$ & 1 & $(0.02)$ & - & & - & \\
\hline 43. Echinoderms & 27 & $(62)$ & 72 & $(19.4)$ & 82 & $(27.4)$ & 29 & $(14.3)$ \\
\hline 44. Holothurians & 4 & $(2.0)$ & 7 & $(2.2)$ & - & & - & \\
\hline 45. Psolus sp. & - & & 1 & $(0.03)$ & - & & - & \\
\hline 46. Cucumaria sp. & 1 & $(0.29)$ & - & & - & & - & \\
\hline 47. Strongvlocentrotus droebachiensis & 1 & $(0.04)$ & 57 & $(2.6)$ & 63 & $(8.1)$ & - & \\
\hline 48. Seastars & 22 & $(4.1)$ & 21 & $(5.2)$ & 11 & $(6.6)$ & 13 & $(2.5)$ \\
\hline 49. Ophiuroids & 3 & $(0.07)$ & 24 & $(9.1)$ & 15 & $(8.7)$ & 4 & $(3.7)$ \\
\hline 50. Ophiopholis aculeata & - & & 9 & $(5.4)$ & 0.3 & $(0.13)$ & 6 & $(5.8)$ \\
\hline 51. Amphiopholis sauamata & 1 & $(0.02)$ & - & & - & & - & \\
\hline 52. Ascidians & 28 & $(12.1)$ & 12 & $(3.1)$ & 0.5 & $(0.19)$ & 0.5 & $(0.28)$ \\
\hline 53. Fish & 21 & $(1.8)$ & 9 & $(2.1)$ & 0.5 & $(0.11)$ & 1 & $(0.27)$ \\
\hline 54. Other animals a & 5 & $(0.86)$ & 3 & $(0.16)$ & - & & - & \\
\hline 55. Unidentified animal tissue & 79 & $(20.1)$ & 69 & $(13.9)$ & 95 & $(27.8)$ & 100 & $(50.2)$ \\
\hline 56. Plants & 83 & $4.2)$ & 49 & $(4.2)$ & 44 & $(2.0)$ & 37 & $(2.6)$ \\
\hline 57. Algae & 77 & $(3.9)$ & 44 & $(3.3)$ & 44 & $(1.9)$ & 37 & $(2.6)$ \\
\hline 58. Brown algae & 36 & $(1.1)$ & 9 & $(2.2)$ & 5 & $(0.12)$ & 7 & $(0.64)$ \\
\hline 59. Green algae & 14 & $(0.46)$ & 1 & $(002)$ & 3 & $(0.08)$ & 4 & $(0.23)$ \\
\hline 60. Red algae & 23 & $(0.06)$ & 21 & $(0.53)$ & 33 & $(1.2)$ & 27 & $(1.3)$ \\
\hline 61. Tufted red algae ${ }^{\text {b }}$ & - & & 14 & $(0.26)$ & 29 & $(1.0)$ & 26 & $(1.2)$ \\
\hline 62. CoralJina officianalis & 36 & $(12)$ & 15 & $(0.40)$ & 9 & $(0.52)$ & 2 & $(0.11)$ \\
\hline 63. Zostera marina & 2 & $(0.04)$ & 1 & $(0.03)$ & - & & - & \\
\hline 64. Gravel & 27 & $(1.5)$ & 38 & $(1.5)$ & 4 & $(0.16)$ & 3 & $(0.10)$ \\
\hline 65. Foreign material c & & & & & & & & \\
\hline & & & & & & & & \\
\hline${ }^{2}$ Eggs, nematodes, nemertines, & & & & & & & & \\
\hline turbellarians & & & & & & & \\
\hline b Family: Ceramiaceae & & & & & & & \\
\hline${ }^{2}$ Plastic, rubber, wood & & & & & & & \\
\hline
\end{tabular}

\begin{tabular}{|c|c|c|}
\hline & $\begin{array}{l}\% \text { volume contribution } \\
\text { main groups }\end{array}$ & $\begin{array}{l}\% \text { volume contribution } \\
\text { to specific group }\end{array}$ \\
\hline Formaniferans & 0.31 & \\
\hline Macroalgae & 3.94 & \\
\hline Coralline algae (Corallina officinalis) & 1.78 & \\
\hline Hydrozoans & 2.07 & \\
\hline Bivalves & 16.57 & \\
\hline Mytilus edulis & & 2.02 \\
\hline Modiolus modiolus & & 9.92 \\
\hline
\end{tabular}




\begin{tabular}{|c|c|c|}
\hline Unidentified Pelecypoda & & 4.63 \\
\hline Gastropods & 5.85 & \\
\hline Lacuna vincta & & 0.28 \\
\hline Unidentified Gastropoda & & 0.57 \\
\hline Polychaetes & 5.97 & \\
\hline Nereidae & & 3.18 \\
\hline Polynoidae & & 2.71 \\
\hline Unidentified Polychaeta & & 0.08 \\
\hline Barnacles (Balanus sp .) & 0.12 & \\
\hline Crustacean meiofauna & 0.53 & \\
\hline Harpacticoida & & 0.03 \\
\hline Ostracoda & & 0.21 \\
\hline Unidentified minute Crustacea & & 0.29 \\
\hline Amphipods & 0.54 & \\
\hline Corophium sp. & & 0.04 \\
\hline Gammarus sp. & & 0.03 \\
\hline Caprellidea & & 0.04 \\
\hline Gammaridae & & 0.16 \\
\hline Unidentified amphipods & & 0.27 \\
\hline Isopods & 0.67 & \\
\hline Idotea sp. & & 0.13 \\
\hline $\begin{array}{r}\text { Idoteidae } \\
\end{array}$ & & 0.19 \\
\hline Unidentified valviferan isopods & & 0.34 \\
\hline Carideans & 0.24 & \\
\hline Crangon septemspinosa & & 0.1 \\
\hline Unidentified carideans & & 0.13 \\
\hline Pagurids & 4.16 & \\
\hline Pagurus acadianus & & 0.51 \\
\hline Paguridae & & 3.65 \\
\hline Rock crab (Cancer irroratus) & 26.37 & \\
\hline $\begin{array}{l}\text { American lobster (Homarus } \\
\text { americanus) }\end{array}$ & 0.76 & \\
\hline Echinoderms & 2.22 & \\
\hline Strongylocentrotus droebachiensis & & 1.02 \\
\hline Ophiuroidea & & 0.12 \\
\hline Unidentified echinoderms & & 1.09 \\
\hline Fish & 0.66 & \\
\hline Flesh & 27.24 & \\
\hline Sum \%s volume contribution & 100 & \\
\hline
\end{tabular}


Wageningen Marine Research

$\mathrm{T}+31(0) 317480900$

E: marine-research@wur.nl

www.wur.eu/marine-research

Visitors' address

- Ankerpark 271781 AG Den Helder

- Korringaweg 7, 4401 NT Yerseke

- Haringkade 1, 1976 CP IJmuiden
With knowledge, independent scientific research and advice, Wageningen Marine Research substantially contributes to more sustainable and more careful management, use and protection of natural riches in marine, coastal and freshwater areas.
Wageningen Marine Research is part of Wageningen University \& Research. Wageningen University \& Research is the collaboration between Wageningen University and the Wageningen Research Foundation and its mission is: 'To explore the potential for improving the quality of life' 